In the format provided by the authors and unedited.

\title{
Total synthesis of brevianamide A
}

Robert C. Godfrey, Nicholas J. Green $\odot$, Gary S. Nichol and Andrew L. Lawrence $₫$

EaStCHEM School of Chemistry, University of Edinburgh, Edinburgh, UK. $\otimes_{e}$-mail: a.lawrence@ed.ac.uk 


\section{SUPPORTING INFORMATION}

\section{Total synthesis of brevianamide A.}

Robert C. Godfrey, Nicholas J. Green, Gary S. Nichol and Andrew L. Lawrence*

EaStCHEM School of Chemistry, University of Edinburgh, Joseph Black Building, David Brewster Road, Edinburgh, UK, EH9 3FJ. E-mail: a.lawrence@ed.ac.uk 


\section{Supporting Information - Table of Contents}

1. Overview of previous total and formal syntheses of brevianamide B............................... 4

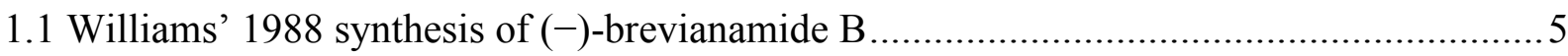

1.2 Williams' 1998 synthesis of $( \pm)$-brevianamide B .................................................... 6

1.3 Williams' 2006 synthesis of $( \pm)$-brevianamide B ................................................ 7

1.4 Williams' 2007 synthesis of $( \pm)$-brevianamide B \& $( \pm)$-dehydrodeoxybreviaminde E .......8

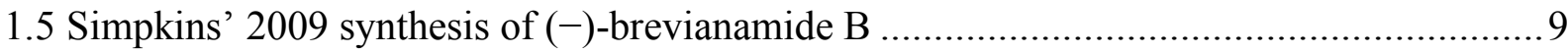

1.6 Scheerer's 2016 formal synthesis of $( \pm)$-brevianamide B ............................................. 10

1.7 Scheerer's 2017 formal synthesis of $( \pm)$-brevianamide B............................................ 11

2. Quantitative analysis of brevianamide A and B in Penicillium brevicompactum .................. 12

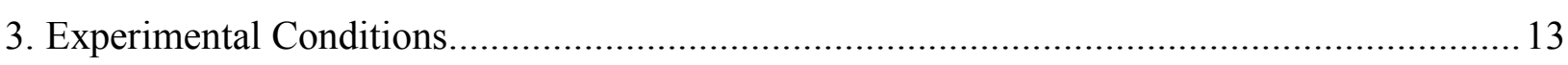

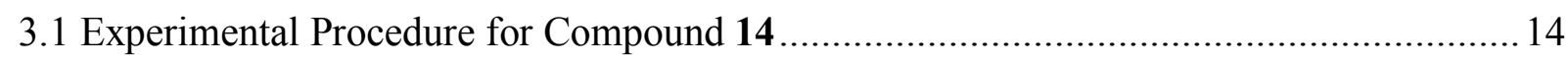

3.2 Experimental Procedure for Compound 15 ............................................................ 15

3.3 Experimental Procedure for Compound 16 ....................................................... 16

3.4 Experimental Procedure for Compound 17 ................................................................. 17

3.5 Experimental Procedure for Compound 19 .......................................................... 18

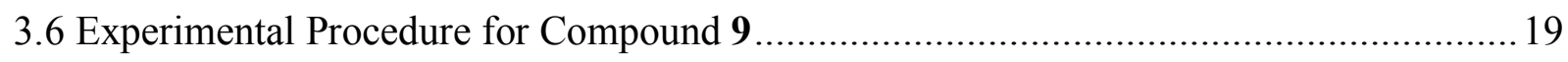

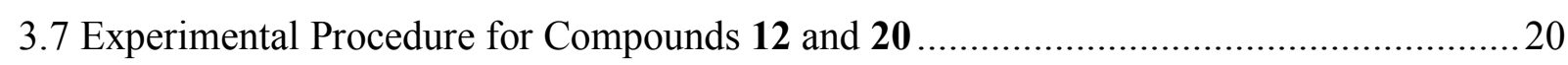

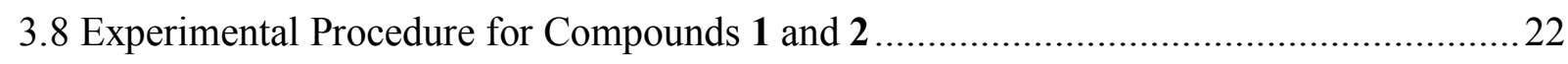

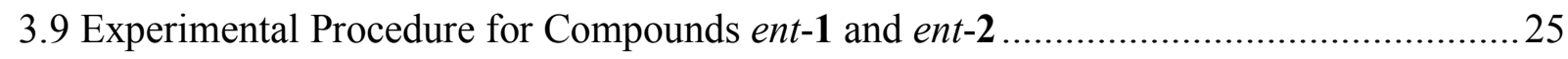

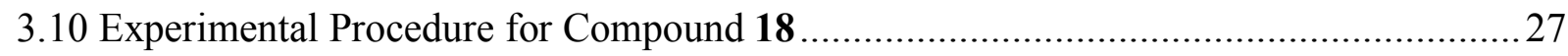

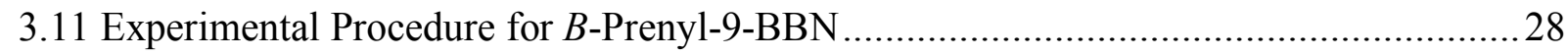

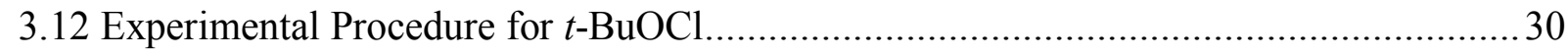

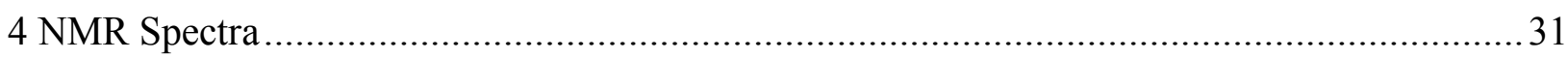

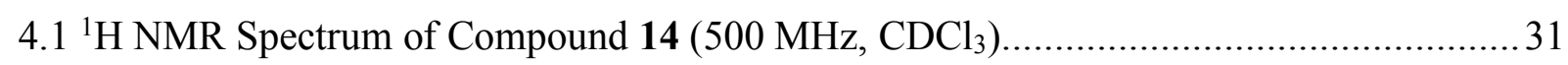

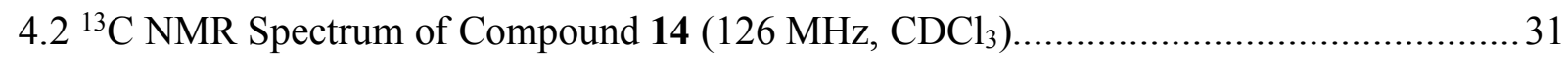

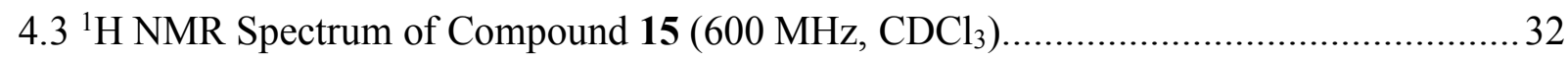

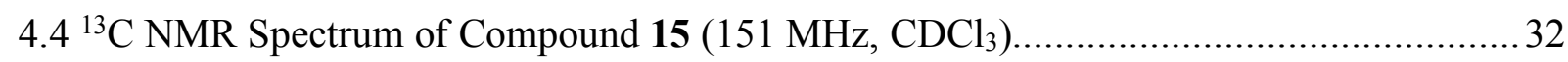

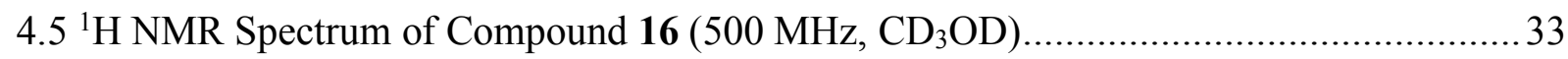

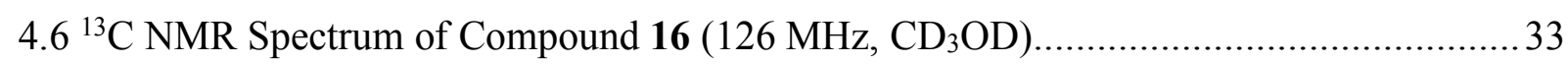

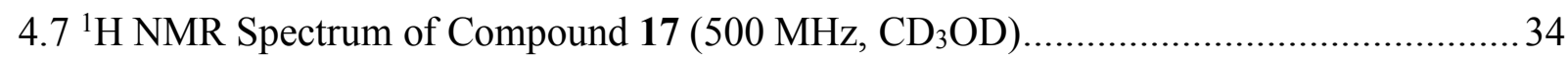

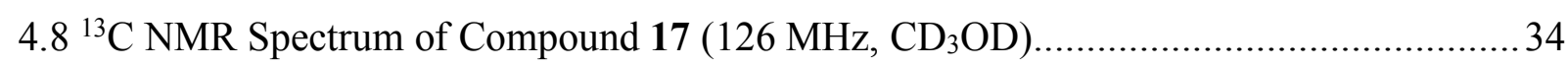

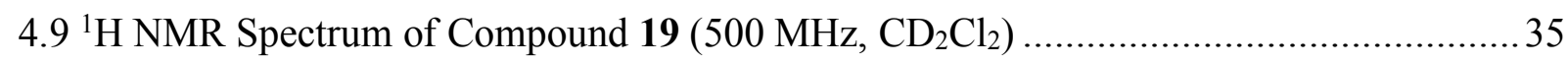

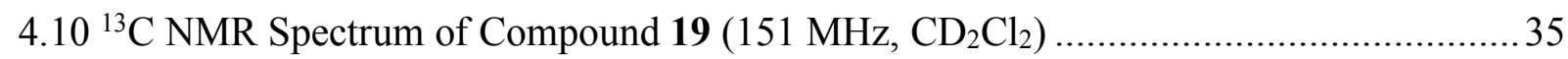

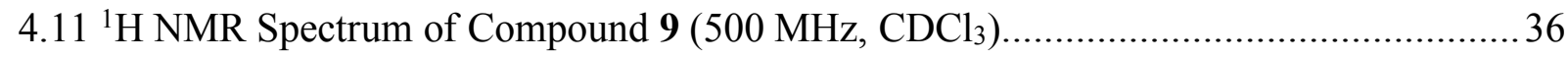

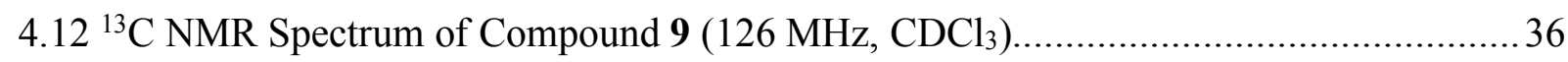

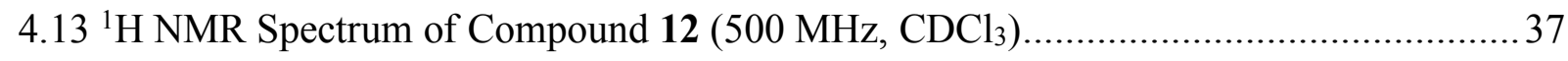

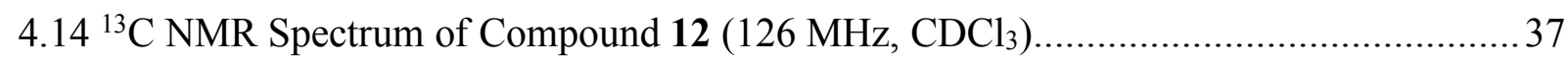

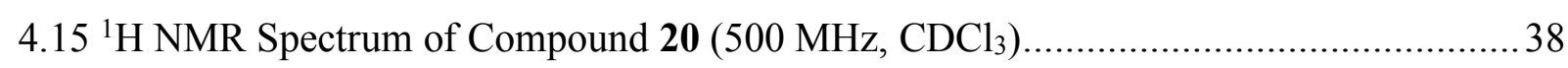

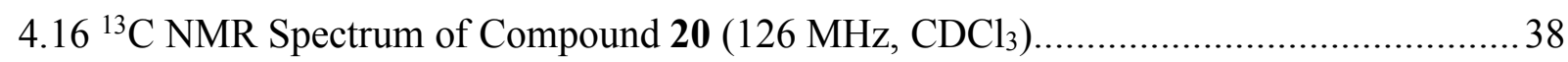


$4.17{ }^{1} \mathrm{H}$ NMR Spectrum of Brevianamide A (1) (500 MHz, $\left.\mathrm{CDCl}_{3}, 3.0 \mathrm{mg} / \mathrm{mL}\right)$ 39

$4.18{ }^{13} \mathrm{C}$ NMR Spectrum of Brevianamide A (1) (126 MHz, $\left.\mathrm{CDCl}_{3}, \sim 3.0 \mathrm{mg} / \mathrm{mL}\right)$. 39

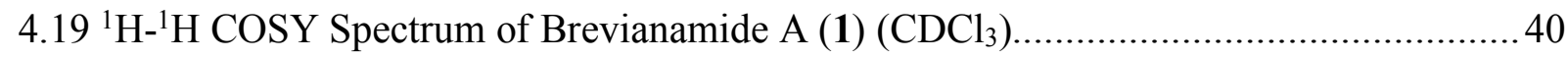

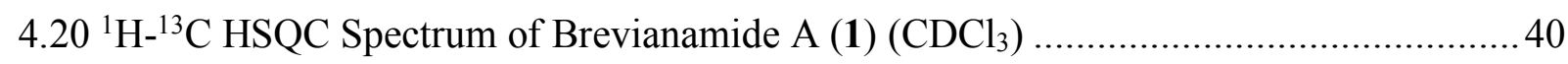

$4.21{ }^{1} \mathrm{H}_{-}{ }^{13} \mathrm{C}$ HMBC Spectrum of Brevianamide A (1) $\left(\mathrm{CDCl}_{3}\right)$.......................................... 41

$4.22{ }^{1} \mathrm{H}-{ }^{1} \mathrm{H}$ NOESY Spectrum of Brevianamide A (1) $\left(\mathrm{CDCl}_{3}\right)$........................................ 41

$4.23{ }^{1} \mathrm{H}$ NMR Spectra of Brevianamide A (1) (500 MHz, $\mathrm{CDCl}_{3}$ - conc. dependence) .......... 42

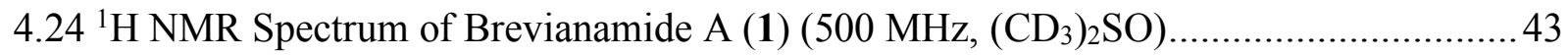

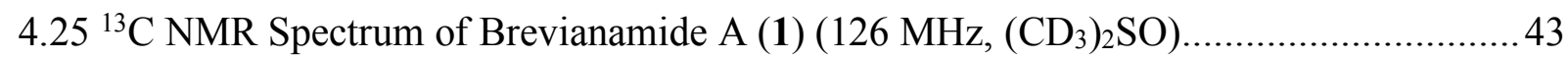

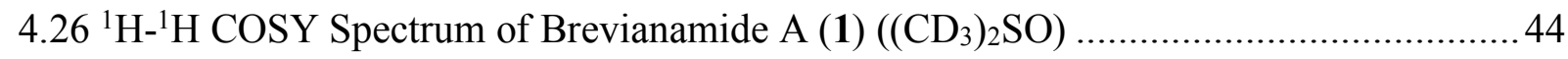

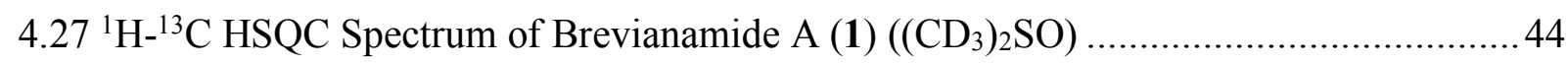

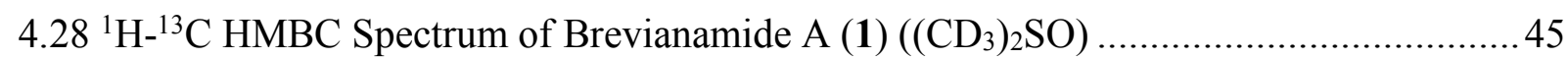

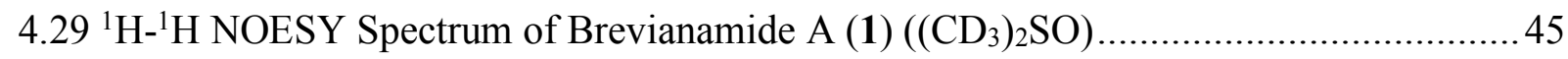

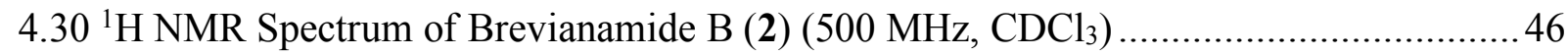

$4.31{ }^{13} \mathrm{C}$ NMR Spectrum of Brevianamide B (2) $\left(126 \mathrm{MHz}, \mathrm{CDCl}_{3}\right)$...................................46

$4.32{ }^{1} \mathrm{H}$ NMR Spectrum of Brevianamide B (2) $\left(500 \mathrm{MHz},\left(\mathrm{CD}_{3}\right)_{2} \mathrm{SO}\right)$............................... 47

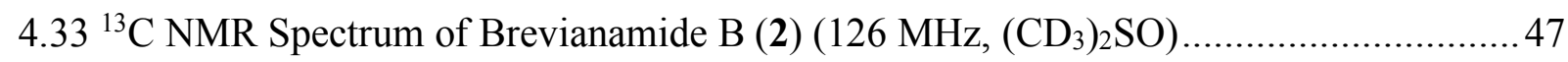

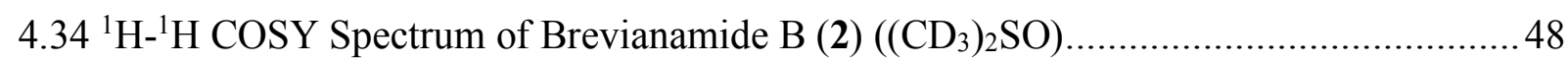

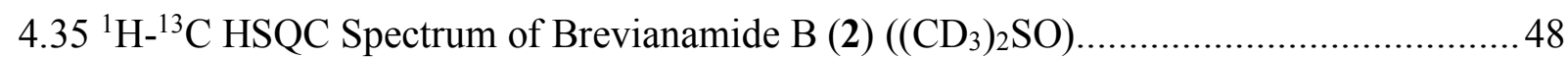

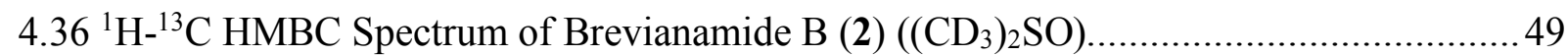

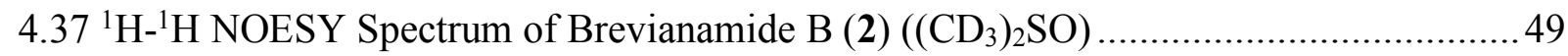

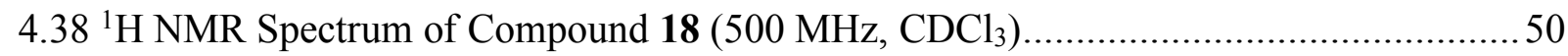

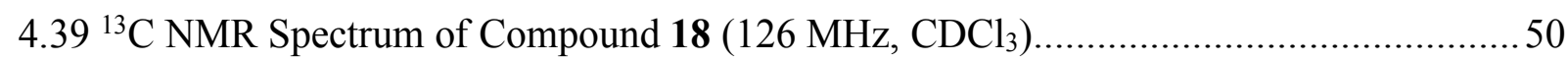

$4.40{ }^{1} \mathrm{H}$ NMR Spectrum of 3-Chloro-3-methyl-1-butyne (500 MHz, $\left.\mathrm{CDCl}_{3}\right)$.......................51

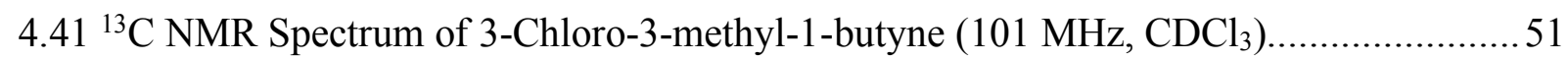

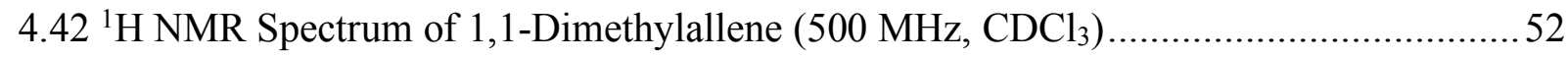

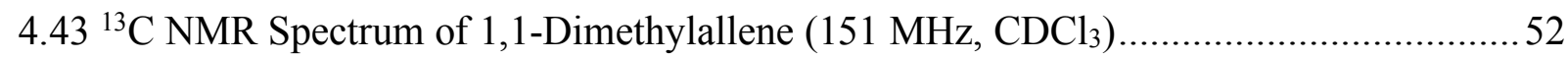

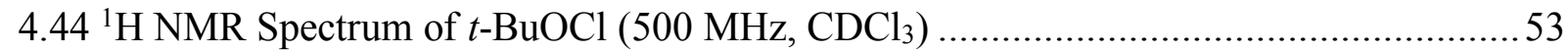

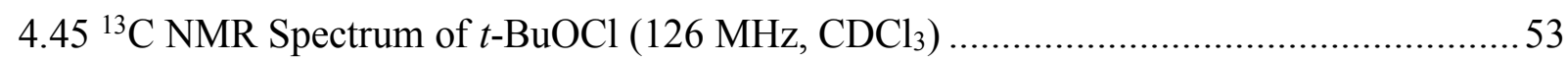

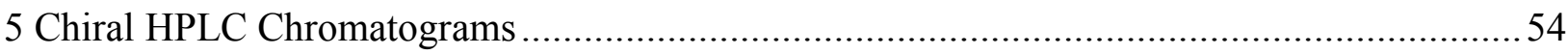

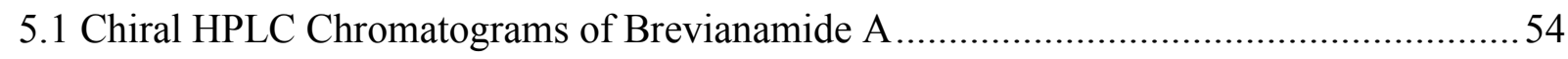

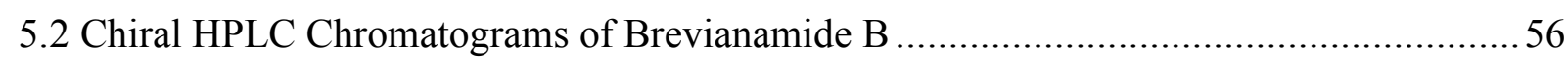

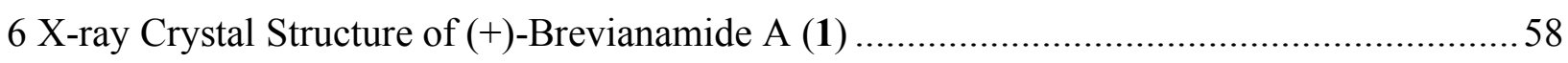


1. Overview of previous total and formal syntheses of brevianamide B.

1.1 Williams' 1988 synthesis of (-)-brevianamide B (cationic cyclization)

- 18 steps

- $1.5-2.5 \%$ overall yield

- $0.8 \mathrm{mg}$ prepared

1.2 Williams' 1998 synthesis of $( \pm)$-brevianamide B (first biomimetic)

- 12 steps

- $2.8 \%$ overall yield

- $7.7 \mathrm{mg}$ prepared

1.3 Williams' 2006 synthesis of $( \pm)$-brevianamide B (late-stage indole formation)

- 12 steps

- $1.4 \%$ overall yield

- $4.0 \mathrm{mg}$ prepared

1.4 Williams' 2007 synthesis of $( \pm)$-brevianamide B (second biomimetic)

- 14 steps

- $0.9 \%$ overall yield

- $4.7 \mathrm{mg}$ prepared

1.5 Simpkins' 2009 synthesis of (-)-brevianamide B (cationic double-cyclization)

- 9 steps

- $1.4 \%$ overall yield

- $6.0 \mathrm{mg}$ prepared

1.6 Scheerer's 2016 formal synthesis of ( \pm )-brevianamide B (first intermolecular [4+2])

- 13 steps $(11+2$ formal steps $)$

- $2.9 \%$ overall yield $(6.4 \% \times 45 \%$ formal steps $)$

- $7.1 \mathrm{mg}$ of final intermediate

1.7 Scheerer's 2017 formal synthesis of $( \pm)$-brevianamide B (second intermolecular $[4+2])$

- 15 steps $(13+2$ formal steps $)$

- $2.1 \%$ overall yield $(4.6 \% \times 45 \%$ formal steps $)$

- $\quad 5.0 \mathrm{mg}$ of final intermediate 


\subsection{Williams' 1988 synthesis of (-)-brevianamide B (cationic cyclization approach)}

- 18 steps

- $1.5-2.5 \%$ overall yield

- $0.8 \mathrm{mg}$ prepared

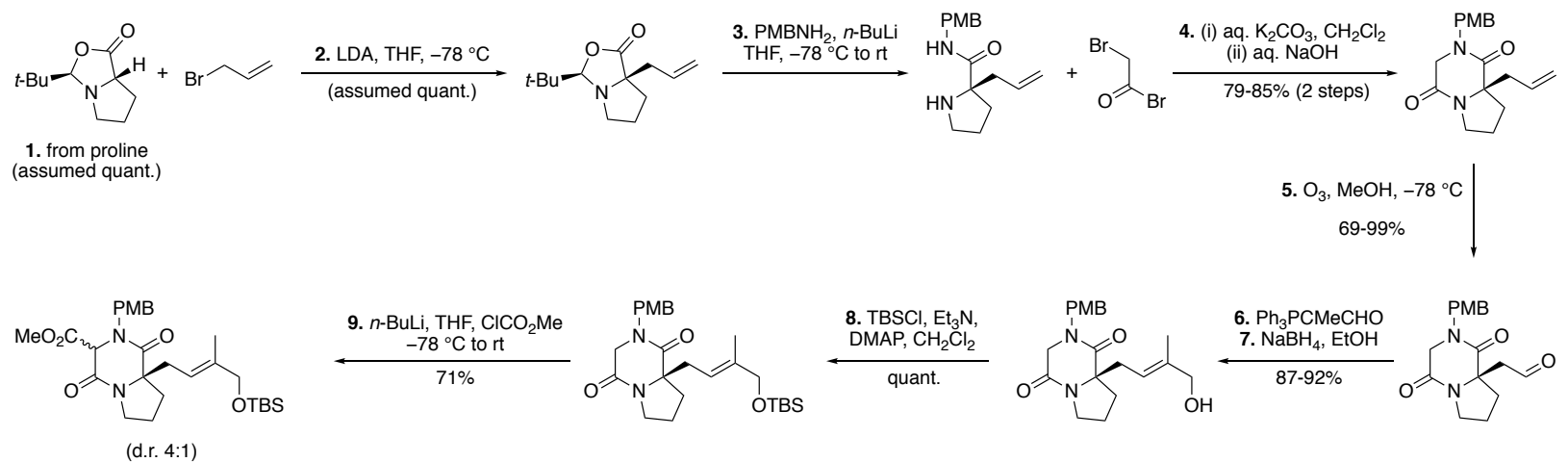

10. $\underset{82{ }_{3}^{\circ} \mathrm{P}, \mathrm{MeCN}}{62 \%}$
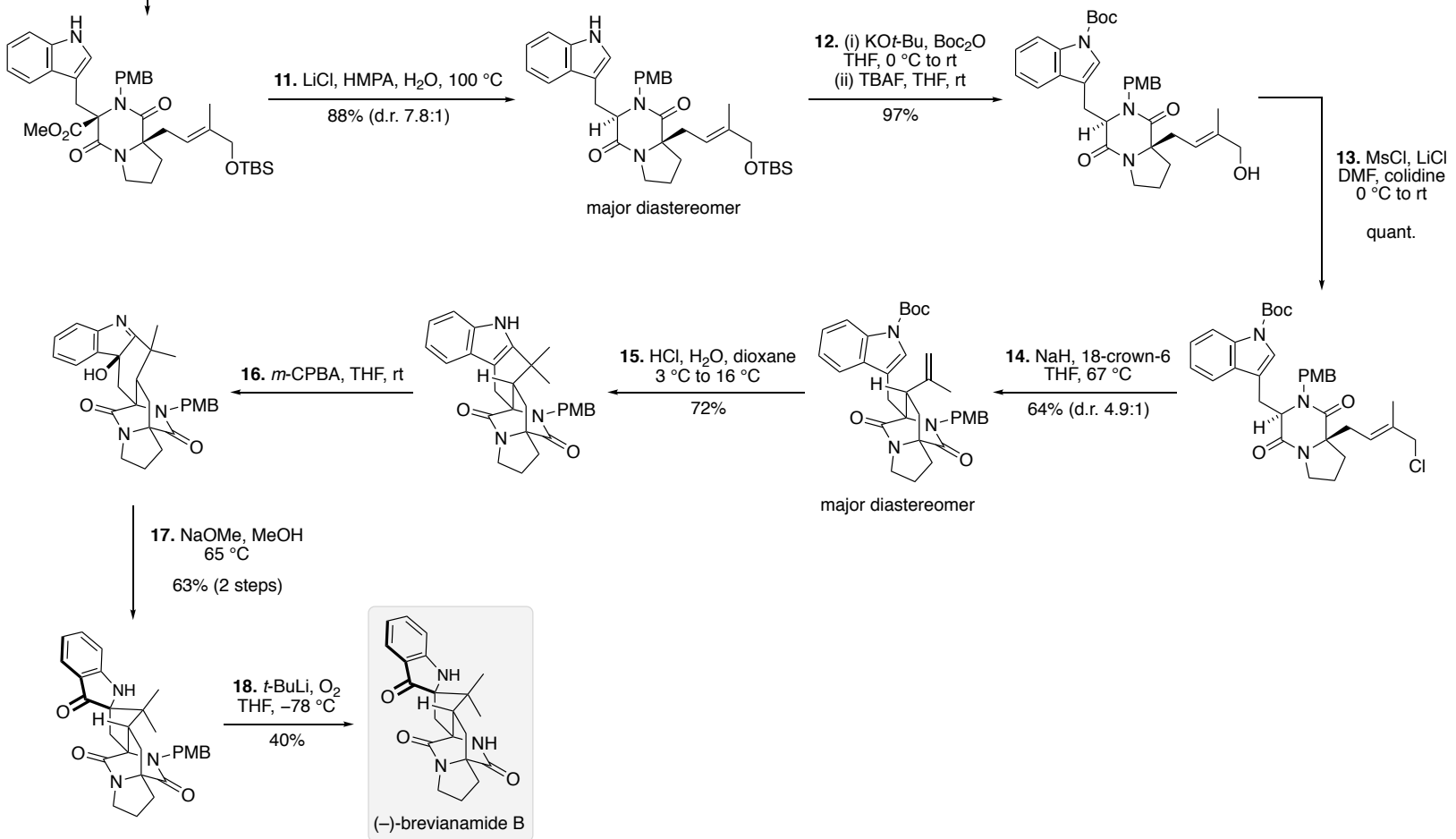

major diastereomer

\section{References:}

1. Williams, R. M., Glinka, T. \& Kwast, E. Facial selectivity of the intramolecular $\mathrm{S}_{\mathrm{N}} 2^{\prime}$ cyclization: stereocontrolled total synthesis of brevianamide B. J. Am. Chem. Soc. 110, 5927 5929 (1988).

2. Williams, R. M. \& Kwast, E. Carbanion-mediated oxidative deprotection of non-enolizable benzylated amides. Tetrahedron Lett. 30, 451-454 (1989)

3. Williams, R. M., Glinka, T., Kwast, E., Coffman, H. \& Stille, J. K. Asymmetric, stereocontrolled total synthesis of (-)-brevianamide B. J. Am. Chem. Soc. 112, 808-821 (1990). 


\subsection{Williams' 1998 synthesis of ( \pm )-brevianamide B (first biomimetic approach)}

- 12 steps

- $2.8 \%$ overall yield

- $7.7 \mathrm{mg}$ prepared

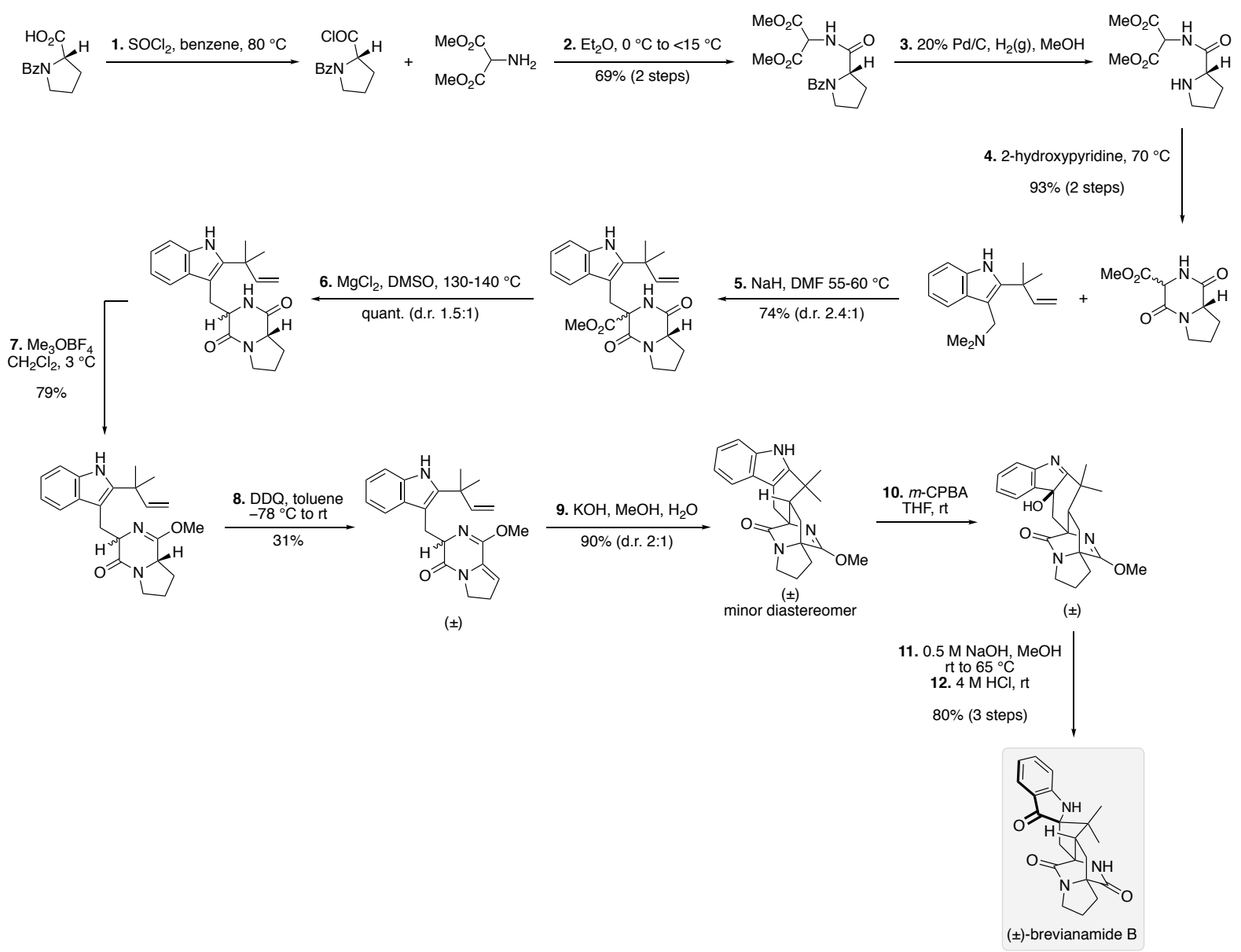

\section{References:}

1. Williams, R. M., Sanz-Cervera, J. F., Sancenón, F., Marco, J. A. \& Halligan, K. Biomimetic Diels-Alder cyclizations for the construction of the brevianamide, paraherquamide sclerotamide, and VM55599 ring systems. J. Am. Chem. Soc. 120, 1090-1091 (1998).

2. Williams, R. M., Sanz-Cervera, J. F., Sancenón, F., Marco, J. A. \& Halligan, K. Biomimetic Diels-Alder cyclizations for the construction of the brevianamide, paraherquamide, sclerotamide, asperparaline and VM55599 ring systems. Bioorg. Med. Chem. 6, 1233-1241 (1998).

3. Steps 1-6 taken from; Kametani, T., Kanaya, N. \& Ihara, M. Studies on the syntheses of heterocyclic compounds. Part 876. The chiral total synthesis of brevianamide $\mathrm{E}$ and deoxybrevianamide E. J. Chem. Soc. Perkin Trans. I 959-963 (1981). 
- 12 steps

- $1.4 \%$ overall yield

- $4.0 \mathrm{mg}$ prepared
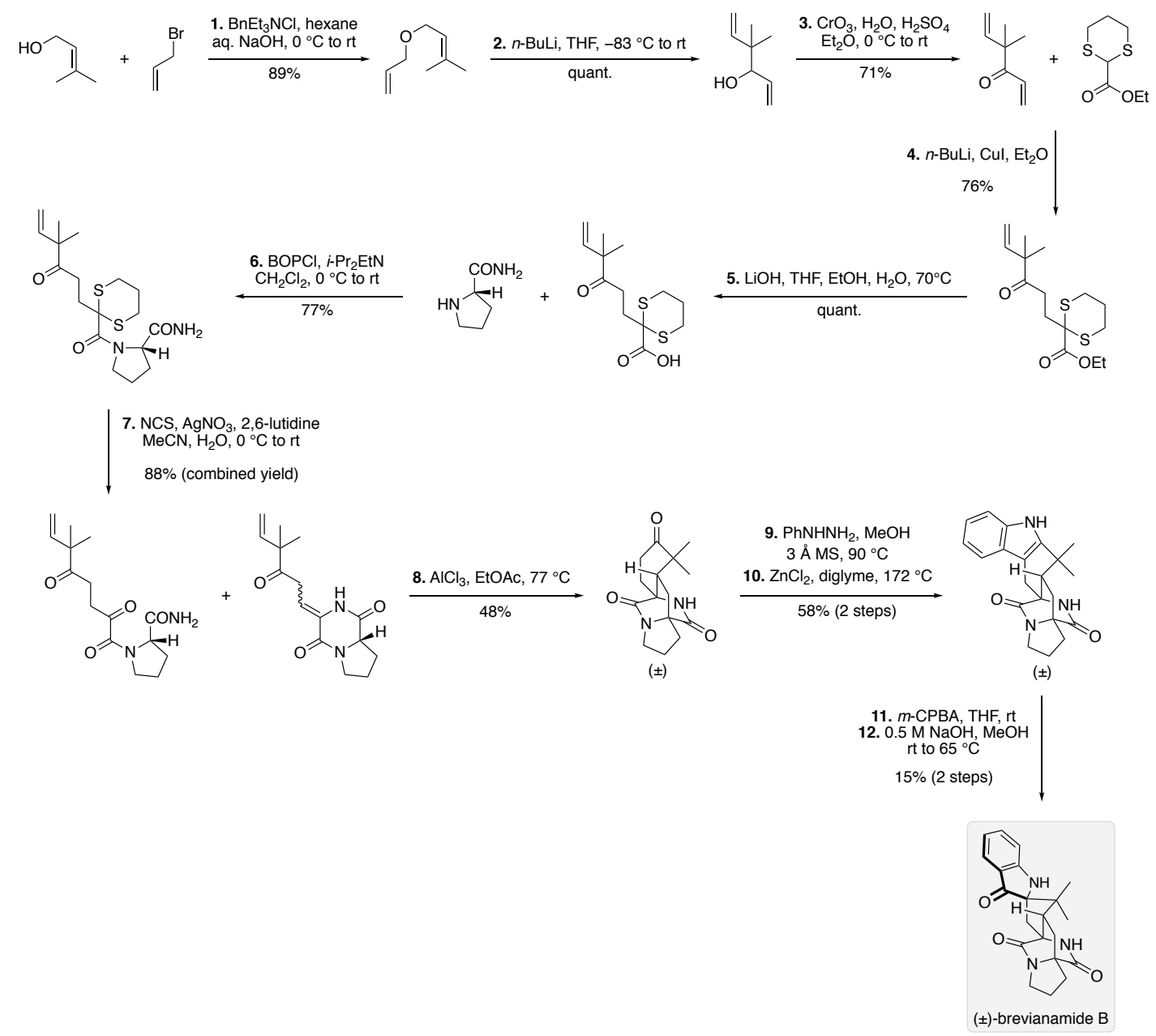

\section{References:}

1. Adams, L. A., Valente, M. W. N. \& Williams, R. M. A concise synthesis of d,1-brevianamide B via a biomimetically-inspired IMDA construction. Tetrahedron 62, 5195-5200 (2006).

2. Steps 1-3 taken from Dauben, W. G., Cogen, J. M., Ganzer, G. A. \& Behar, V. Photochemistry of 1,5-hexadien-3-ones: Wavelength-dependent selectivity in intramolecular enone-olefin photoadditions. J. Am. Chem. Soc. 113, 5817-5824 (1991). 


\subsection{Williams' 2007 synthesis of $( \pm)$-brevianamide B \& $( \pm)$-dehydrodeoxybreviaminde E}

(second biomimetic approach)

$( \pm)$-brevianamide B

- 14 steps

- $0.9 \%$ overall yield

- $4.7 \mathrm{mg}$ prepared

$( \pm)$-dehydrodeoxybreviaminde $\mathrm{E}$

- 12 steps

- $8 \%$ overall yield

- $98 \mathrm{mg}$ prepared
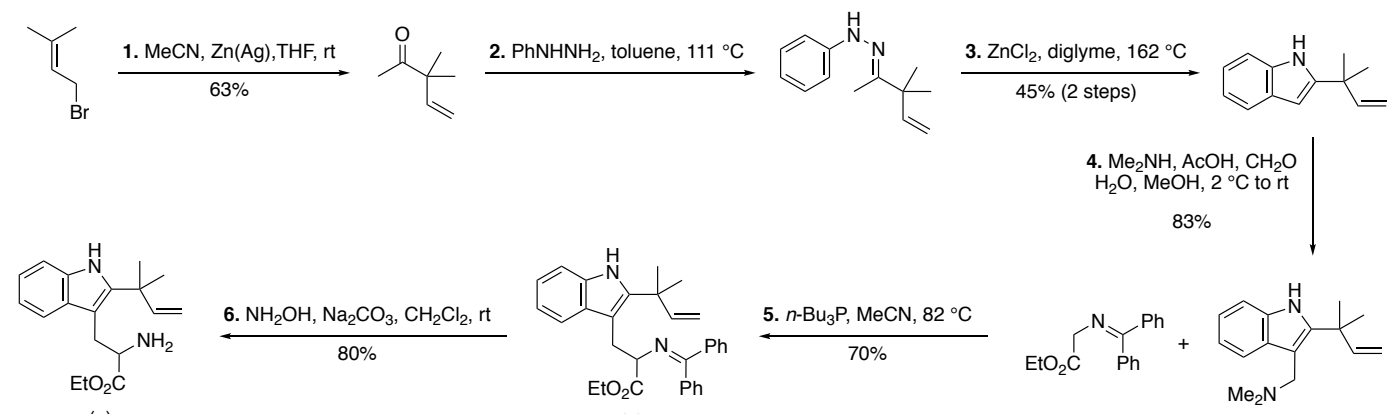

7. $\mathrm{Boc}_{2} \mathrm{O}, \mathrm{NaOH}$, dioxane, $\mathrm{rt}$ 8. $\mathrm{LiOH}, \mathrm{THF}, \mathrm{H}_{2} \mathrm{O}$, r

$78 \%$ (2 steps)

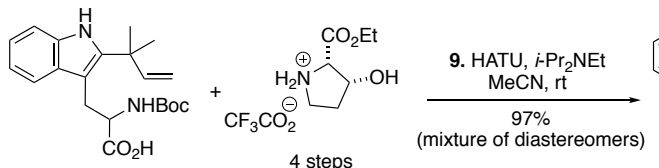

$( \pm)$

4 steps
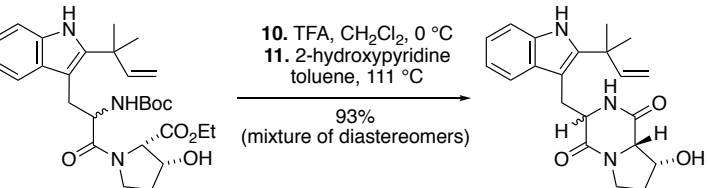

$( \pm)$
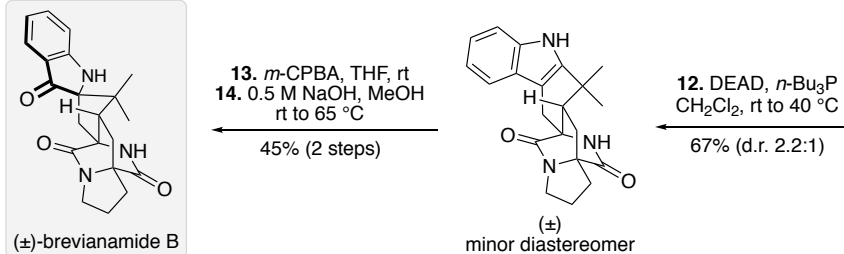

$67 \%$ (d.r. 2.2:1)

(土)

12. DEAD, $n-\mathrm{Bu}_{3} \mathrm{P}$ $\mathrm{CH}_{2} \mathrm{Cl}_{2}$, rt

$86 \%$

minor diastereomer

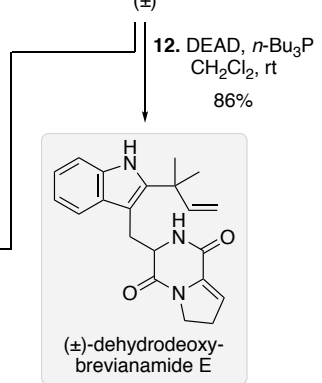

\section{References:}

1. Greshock, T. J. \& Williams, R. M. Improved biomimetic total synthesis of d,1-stephacidin A. Org. Lett. 9, 4255-4258 (2007).

2. Steps 1-4 taken from; Sanz-Cervera, J. F., Glinka, T. \& Williams, R. M. Biosynthesis of brevianamides A and B: In search of the biosynthetic Diels-Alder construction. Tetrahedron 49, 8471-8482 (1993). (which used ${ }^{3} \mathrm{H}_{2} \mathrm{CO}$ in step 4)

3. Steps 5-8 taken from; Stocking, E. M., Sanz-Cervera, J. F. \& Williams, R. M. Total Synthesis of VM55599. Utilization of an Intramolecular Diels-Alder Cycloaddition of Potential Biogenetic Relevance. J. Am. Chem. Soc. 122, 1675-1683 (2000). 
- 9 steps

- $1.4 \%$ overall yield

- $6.0 \mathrm{mg}$ prepared
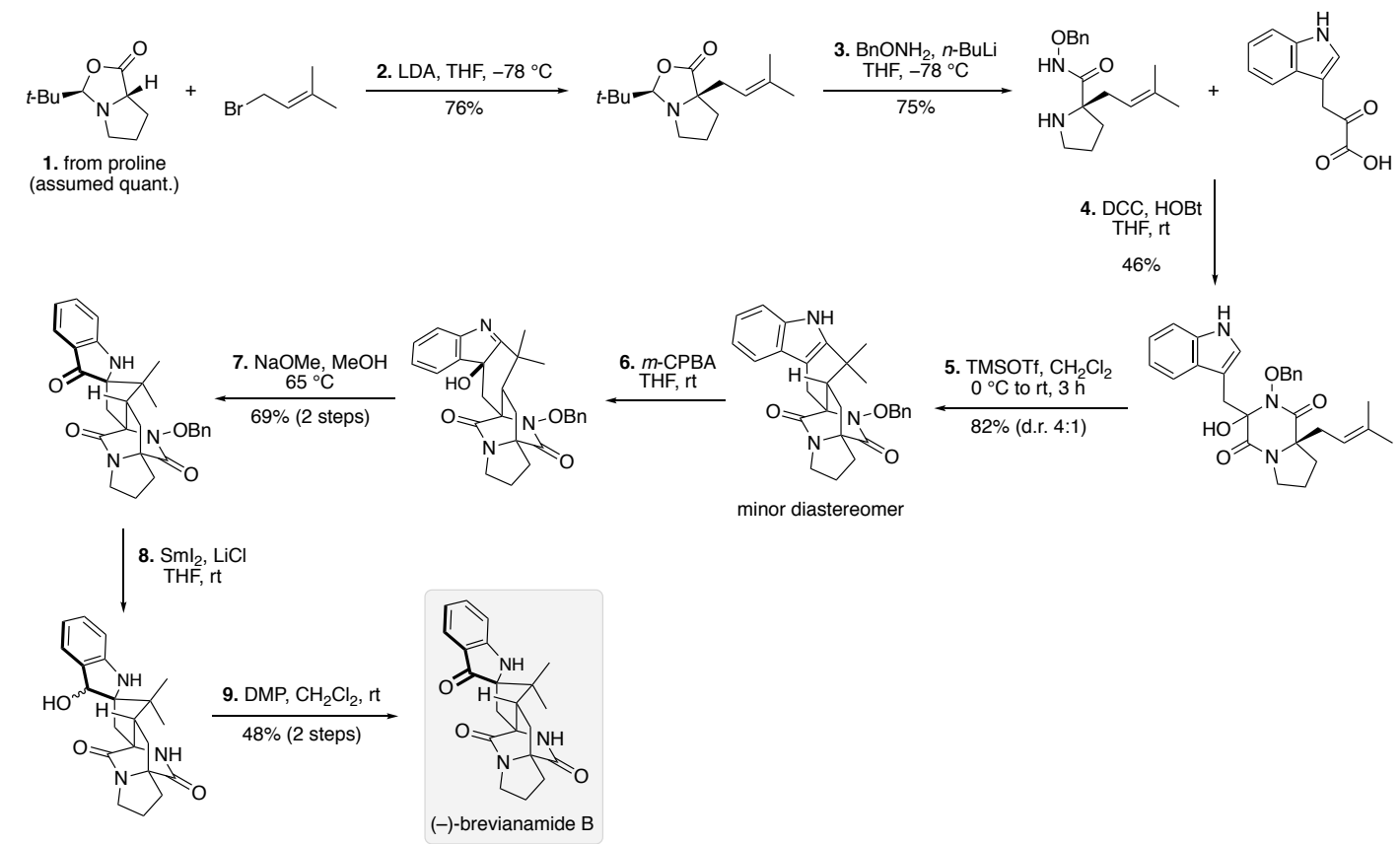

\section{References:}

1. Frebault, F. C., Simpkins, N. S. \& Fenwick, A. A concise enantioselective synthesis of entmalbrancheamide B. J. Am. Chem. Soc. 131, 4214-4215 (2009).

2. Frebault, F. C. \& Simpkins, N. S. A cationic cyclisation route to prenylated indole alkaloids: synthesis of malbrancheamide $\mathrm{B}$ and brevianamide $\mathrm{B}$, and progress towards stephacidin $\mathrm{A}$. Tetrahedron 66, 6585-6596 (2010). 


\subsection{Scheerer's 2016 formal synthesis of ( \pm )-brevianamide B (first intermolecular [4+2]}

approach)

- 13 steps $(11+2$ formal steps $)$

- $2.9 \%$ overall yield $(6.4 \% \times 45 \%$ formal steps $)$

- $7.1 \mathrm{mg}$ of final intermediate

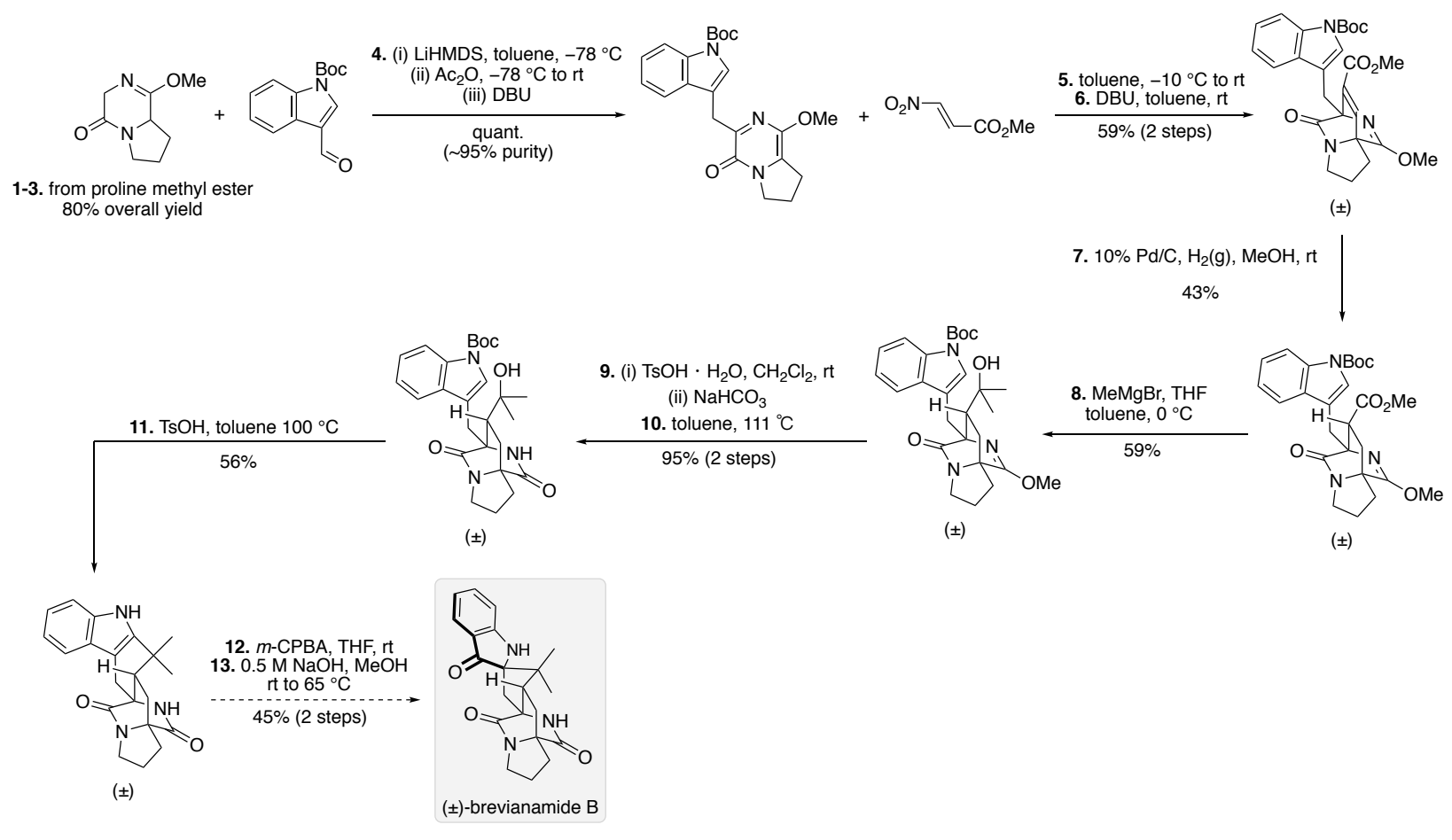

\section{References:}

1. Robins, J. G., Kim, K. J., Chinn, A. J., Woo, J. S. \& Scheerer, J. R. Intermolecular Diels-Alder Cycloaddition for the Construction of Bicyclo[2.2.2]diazaoctane Structures: Formal Synthesis of Brevianamide B and Premalbrancheamide. J. Org. Chem. 81, 2293-2301 (2016).

2. Steps 1-3 taken from; Laws, S. W. \& Scheerer, J. R. Enantioselective Synthesis of (+)Malbrancheamide B. J. Org. Chem. 78, 2422-2429 (2013).

3. Steps 12-13 taken from; Greshock, T. J. \& Williams, R. M. Improved biomimetic total synthesis of d,1-stephacidin A. Org. Lett. 9, 4255-4258 (2007). 


\subsection{Scheerer's 2017 formal synthesis of $( \pm)$-brevianamide B (second intermolecular [4+2] approach)}

- 15 steps $(13+2$ formal steps $)$

- $2.1 \%$ overall yield $(4.6 \% \times 45 \%$ formal steps $)$

- $5.0 \mathrm{mg}$ of final intermediate
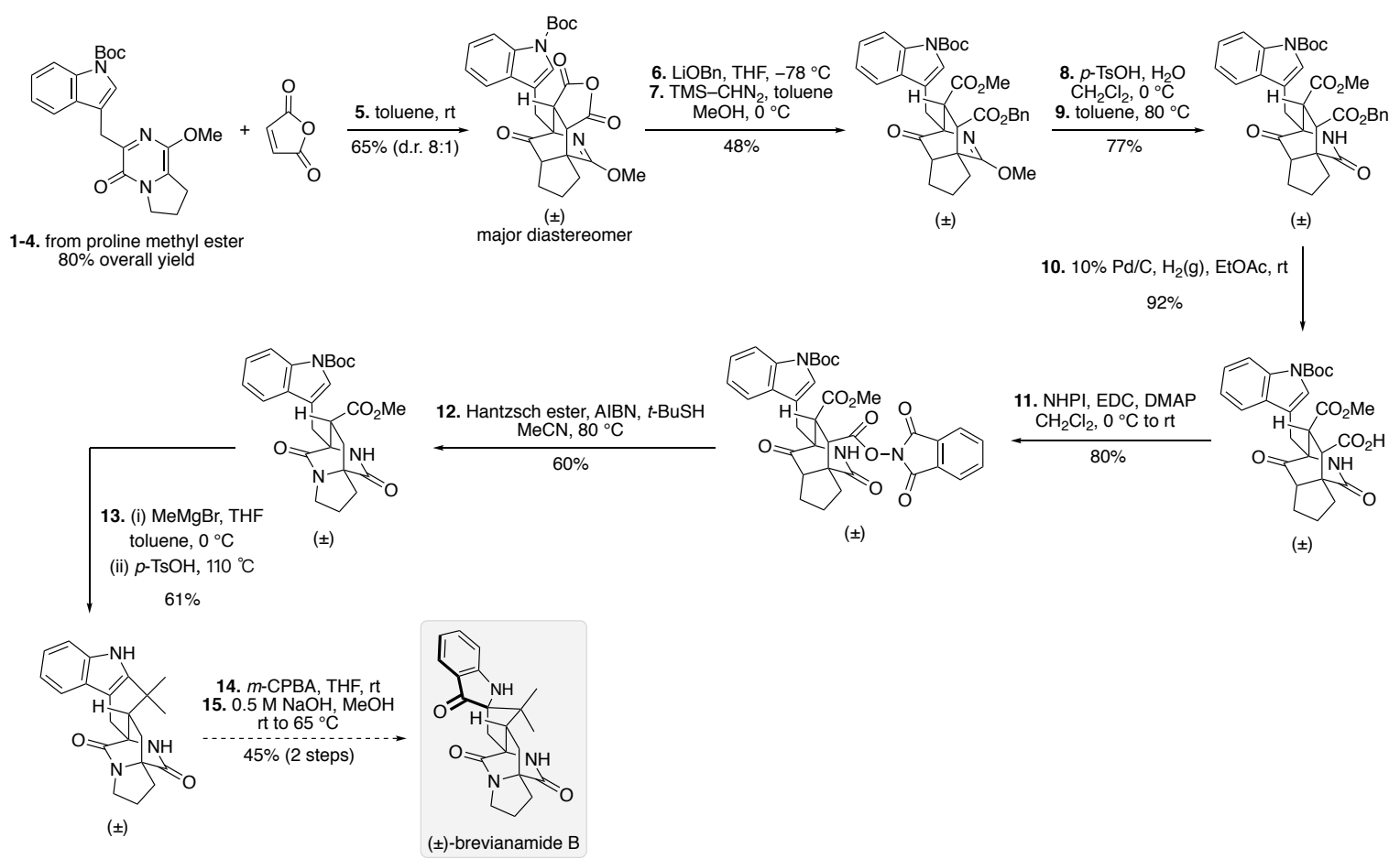

\section{References:}

1. Perkins, J. C., Wang, X., Pike, R. D. \& Scheerer, J. R. Further investigation of the intermolecular Diels-Alder cycloaddition for the synthesis of bicyclo[2.2.2]diazaoctane alkaloids. J. Org. Chem. 82, 13656-13662 (2017).

2. Steps 1-3 taken from; Laws, S. W. \& Scheerer, J. R. Enantioselective Synthesis of (+)Malbrancheamide B. J. Org. Chem. 78, 2422-2429 (2013).

3. Step 4 taken from; Robins, J. G., Kim, K. J., Chinn, A. J., Woo, J. S. \& Scheerer, J. R. Intermolecular Diels-Alder Cycloaddition for the Construction of Bicyclo[2.2.2]diazaoctane Structures: Formal Synthesis of Brevianamide B and Premalbrancheamide. J. Org. Chem. 81, 2293-2301 (2016).

4. Steps 14-15 taken from; Greshock, T. J. \& Williams, R. M. Improved biomimetic total synthesis of d,1-stephacidin A. Org. Lett. 9, 4255-4258 (2007). 


\section{Quantitative analysis of brevianamide A and B in Penicillium brevicompactum}

The ratio of brevianamide A and B in Penicillium brevicompactum cultures was determined to be consistently $\gtrsim 90: 10$ by Campbell and co-workers (see tables below). ${ }^{1}$

Supplementary Table 1. Brevianamide A and B production from $P$. brevicompactum colony growth: over culture, inoculum $4.8 \times 10^{6}$ spores per plate.

\begin{tabular}{cccc}
\hline $\begin{array}{c}\text { Time after inoculation } \\
\text { (h) }\end{array}$ & $\begin{array}{c}\text { Brevianamide A } \\
\text { (mg per plate) }\end{array}$ & $\begin{array}{c}\text { Brevianamide B } \\
\text { (mg per plate) }\end{array}$ & $\mathbf{A}: \mathbf{B}$ \\
\hline 72 & 0.07 & 0.01 & $87.5: 12.5$ \\
84 & 0.19 & 0.02 & $90.5: 9.5$ \\
96 & 0.21 & 0.02 & $91.3: 8.7$ \\
108 & 0.45 & 0.03 & $93.9: 6.1$ \\
120 & 0.42 & 0.04 & $91.3: 8.7$ \\
144 & 0.50 & 0.04 & $92.6: 7.4$ \\
168 & 0.52 & 0.04 & $92.9: 7.1$ \\
192 & 0.53 & 0.05 & $91.4: 8.6$ \\
216 & 0.49 & 0.04 & $92.5: 7.5$
\end{tabular}

Supplementary Table 2. Brevianamide A and B production from P. brevicompactum colony growth: over culture without medium replacement, inoculum $3.6 \times 10^{6}$ spores per plate.

\begin{tabular}{cccc}
\hline $\begin{array}{c}\text { Time after inoculation } \\
\text { (h) }\end{array}$ & $\begin{array}{c}\text { Brevianamide A } \\
\text { (mg per plate) }\end{array}$ & $\begin{array}{c}\text { Brevianamide B } \\
\text { (mg per plate) }\end{array}$ & $\mathbf{A}: \mathbf{B}$ \\
\hline 72 & 0.18 & 0.02 & $90: 10$ \\
84 & 0.28 & 0.03 & $90.3: 9.7$ \\
96 & 0.14 & 0.01 & $93.3: 6.7$ \\
108 & 0.19 & 0.02 & $90.5: 9.5$ \\
120 & 0.67 & 0.06 & $91.8: 8.2$ \\
144 & 0.66 & 0.06 & $91.7: 8.3$
\end{tabular}

Supplementary Table 3. Brevianamide A and B production from $P$. brevicompactum colony growth: over culture with continual medium replacement, inoculum $3.6 \times 10^{6}$ spores per plate.

\begin{tabular}{cccc}
\hline $\begin{array}{c}\text { Time after inoculation } \\
\text { (h) }\end{array}$ & $\begin{array}{c}\text { Brevianamide A } \\
\text { (mg per plate) }\end{array}$ & $\begin{array}{c}\text { Brevianamide B } \\
\text { (mg per plate) }\end{array}$ & $\mathbf{A}: \mathbf{B}$ \\
\hline 72 & 0.12 & 0.01 & $92.3: 7.7$ \\
84 & 0.54 & 0.04 & $93.1: 6.9$ \\
96 & 0.84 & 0.08 & $91.3: 8.7$ \\
108 & 1.08 & 0.09 & $92.3: 7.7$ \\
120 & 1.27 & 0.13 & $90.7: 1.3$ \\
144 & 1.61 & 0.14 & $92: 8$
\end{tabular}

\section{References:}

1. Bird, B. A. \& Campbell, I. M. Brevianamides A and B are formed only after conidiation has begun in solid cultures of Penicillium brevicompactum. Appl. Environ. Microbiol. 42, 521-525 (1981). 


\section{Experimental Conditions}

NMR Spectroscopy: ${ }^{1} \mathrm{H}$ NMR spectra were recorded at $600 \mathrm{MHz}$ on a Bruker 600 spectrometer with a AVANCE $3 \mathrm{HD}$ console, at $500 \mathrm{MHz}$ on Ascend 500 spectrometers with AVANCE 3 and AVANCE 3HD consoles, and at $400 \mathrm{MHz}$ on a Bruker 400 spectrometer with AVANCE 3 console. Residual solvent peaks were used as an internal reference for ${ }^{1} \mathrm{H} N M R$ spectra $\left(\mathrm{CDCl}_{3} \delta 7.26 \mathrm{ppm}\right.$, $\mathrm{CD}_{3} \mathrm{OD} \delta 3.31 \mathrm{ppm}, \mathrm{CD}_{2} \mathrm{Cl}_{2} \delta 5.32 \mathrm{ppm}$, and $\left.\left(\mathrm{CD}_{3}\right)_{2} \mathrm{SO} \delta 2.50 \mathrm{ppm}\right)$. Coupling constants $(J)$ are quoted to the nearest $0.1 \mathrm{~Hz} .{ }^{13} \mathrm{C}$ NMR spectra were recorded at $151 \mathrm{MHz}$ on a Bruker 600 spectrometer with an AVANCE 3HD console, $126 \mathrm{MHz}$ on Ascend 500 spectrometers with AVANCE 3 and AVANCE 3HD consoles, and at $101 \mathrm{MHz}$ on a Bruker 400 spectrometer with AVANCE 3 console. Solvent peaks were used as an internal reference for ${ }^{13} \mathrm{C}$ NMR spectra $\left(\mathrm{CDCl}_{3} \delta 77.16 \mathrm{ppm}, \mathrm{CD}_{3} \mathrm{OD} \delta 49.00 \mathrm{ppm}, \mathrm{CD}_{2} \mathrm{Cl}_{2} \delta 54.00 \mathrm{ppm}\right.$, and $\left.\left(\mathrm{CD}_{3}\right)_{2} \mathrm{SO} \delta 39.52 \mathrm{ppm}\right)$. Assignment of ${ }^{1} \mathrm{H}$ and ${ }^{13} \mathrm{C}$ NMR signals was assisted by ${ }^{1} \mathrm{H}-{ }^{1} \mathrm{H}$ COSY, ${ }^{1} \mathrm{H}-{ }^{13} \mathrm{C}$ HSQC,${ }^{1} \mathrm{H}-{ }^{13} \mathrm{C}$ $\mathrm{HMBC}$ and ${ }^{1} \mathrm{H}-{ }^{1} \mathrm{H}$ NOESY experiments.

IR Spectroscopy: IR spectra were recorded as neat samples on a Shimadzu IR affinity-1 FTIR spectrometer fitted with an ATR attachment.

Mass Spectroscopy: EI mass spectra were recorded on a MAT 900 XP double focussing high resolution sector, run at $70 \mathrm{eV}$. ESI spectra were recorded on a Bruker microTOF, calibrated with sodium formate clusters, with data analysis using Data Analysis 4.1 (Bruker Daltonics).

Analytical TLC: TLC analyses were performed on Merck silica plates coated with silica gel 60 F254 $(0.2 \mathrm{~mm})$ and were visualised with UV light and by staining with $p$-anisaldehyde or $\mathrm{KMnO}_{4}$ standard TLC stain solutions, followed by heating.

Flash Chromatography: Flash chromatography was performed using Merck silica gel 60 (40-63 $\mu \mathrm{m})$ for routine separations and Fluorochem silica gel $60(20-45 \mu \mathrm{m})$ for more challenging separations. Neutralised silica refers to columns packed with solvent containing $1 \% \mathrm{NEt}_{3}$ and rinsed with between 1 and 2 column volumes of solvent (free of $\mathrm{NEt}_{3}$ ) before use.

Optical Rotation: Optical rotations were recorded using a Bellingham Stanley ADP450 polarimeter with a Bellingham Stanley $0.5 \mathrm{~mL}$ cell $(l=0.25 \mathrm{dm})$. Concentrations $(c)$ are reported in $\mathrm{g} / 100 \mathrm{~mL}$.

Chiral HPLC: Analytical chiral HPLC was conducted using a modular Shimadzu system using a LC-20AD pump, DGU-20A 5 R degassing unit, SIL-20A HT autosampler, CTO-20A column oven, SPD-20A UV/Vis detector and CDM-20A communications module.

Experimental Procedures, Reagents and Solvents: Unless stated otherwise, reactions were performed under a positive pressure of dry nitrogen using anhydrous solvents. Commercially available chemicals were used as received, unless specified otherwise. Solvents and reagents dried over $4 \AA$ molecular sieves were dried for at least $24 \mathrm{~h}$ before use. Distillates were collected in flasks cooled with a dry ice/acetone bath to avoid loss of material. For reactions performed under anhydrous reaction conditions the reaction vessels were dried with a heat gun under vacuum prior to use. For anhydrous reactions, $\mathrm{NEt}_{3}$ was dried over activated $4 \AA$ molecular sieves for at least $24 \mathrm{~h}$ before use. 

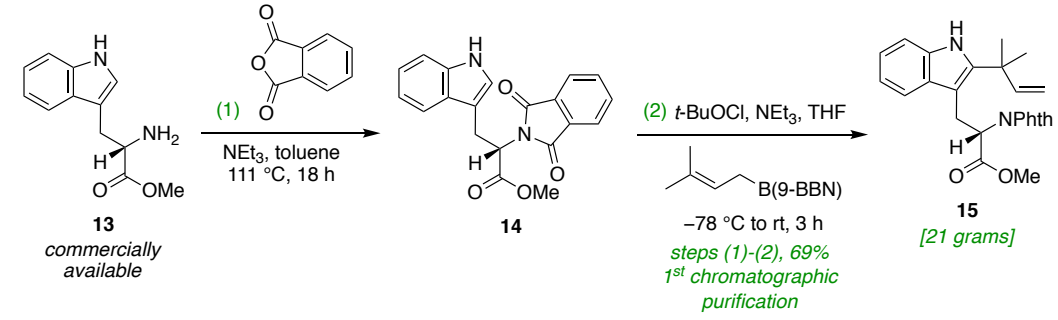

Step (1) - Based on conditions reported by Vederas and co-workers. ${ }^{1}$ To a stirred suspension of L-tryptophan methyl ester hydrochloride $(25.0 \mathrm{~g}, 98.2 \mathrm{mmol})$ and phthalic anhydride $(14.5 \mathrm{~g}$, $98.2 \mathrm{mmol})$ in toluene $(1000 \mathrm{~mL})$ was added $\mathrm{NEt}_{3}(27.4 \mathrm{~mL}, 196 \mathrm{mmol})$ and the mixture heated at reflux for $18 \mathrm{~h}$. The reaction was allowed to cool to room temperature and a mixture of saturated aq. $\mathrm{NH}_{4} \mathrm{Cl}(150 \mathrm{~mL})$ and water $(150 \mathrm{~mL})$ was added. The organic phase was separated, washed with brine $(300 \mathrm{~mL})$, dried $\left(\mathrm{Na}_{2} \mathrm{SO}_{4}\right)$ and concentrated under reduced pressure to yield crude $\mathbf{1 4}$ (33.6 g, 98\% crude mass recovery) which was used without further purification in the next step. A small sample was purified by flash chromatography (2:3 EtOAc/petroleum spirit) to give compound $\mathbf{1 4}$ as a pale-yellow foam suitable for characterisation. All data for compound 14 matched literature values. ${ }^{1}$

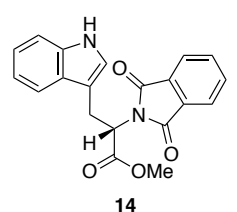

$\mathbf{R}_{\mathbf{f}} 0.24$ (2:3 EtOAc/petroleum spirit)

${ }^{1} \mathbf{H}$ NMR $\left(500 \mathrm{MHz}, \mathrm{CDCl}_{3}\right) \delta 7.97$ (br. s, $\left.1 \mathrm{H}\right), 7.74$ (dd, $\left.J=5.5,3.1 \mathrm{~Hz}, 2 \mathrm{H}\right), 7.65$ (dd, $J=5.5$, $3.0 \mathrm{~Hz}, 2 \mathrm{H}$ ), 7.60 (app. dt, $J=7.9,0.9 \mathrm{~Hz}, 1 \mathrm{H}$ ), 7.25 (app. dt, $J=7.3,0.9,0.9 \mathrm{~Hz}, 1 \mathrm{H}$ ), 7.12 (ddd, $J=8.1,7.0,1.2 \mathrm{~Hz}, 1 \mathrm{H}), 7.05(\mathrm{ddd}, J=8.0,7.0,1.1 \mathrm{~Hz}, 1 \mathrm{H}), 6.99(\mathrm{~d}, J=2.3 \mathrm{~Hz}, 1 \mathrm{H}), 5.28(\mathrm{dd}, J$ $=9.9,6.1 \mathrm{~Hz}, 1 \mathrm{H}), 3.79(\mathrm{~s}, 3 \mathrm{H}), 3.78-3.70(\mathrm{~m}, 2 \mathrm{H}) \mathrm{ppm}$;

${ }^{13} \mathbf{C}$ NMR $\left(126 \mathrm{MHz}, \mathrm{CDCl}_{3}\right) \delta 169.8,167.7,136.2,134.1,131.8,127.3,123.5,122.7,122.2$, 119.6, 118.6, 111.2, 111.2, 53.0, 52.7, 24.9 ppm;

IR (film, cm cm $^{-1}$ 3404, 3057, 3013, 2953, 2924, 2849, 1775, 1740, 1705, 1612, 1555;

HRMS $\left(\mathrm{ESI}^{+}\right.$) $\mathrm{m} / z 349.1211$ (calculated $[\mathrm{M}+\mathrm{H}]^{+}$349.1183), 371.1033 (calculated $[\mathrm{M}+\mathrm{Na}]^{+}$ 371.1002)

$[\boldsymbol{\alpha}]_{D}^{23.3}-201.1^{\circ}\left(c 1.07, \mathrm{CHCl}_{3}\right)$

\section{Reference:}

1. Liu, H., Pattabiraman, V. R. \& Vederas, J. C. Stereoselective Syntheses of 4-Oxa Diaminopimelic Acid and Its Protected Derivatives via Aziridine Ring Opening. Org. Lett. 9, 4211-4214 (2007). 

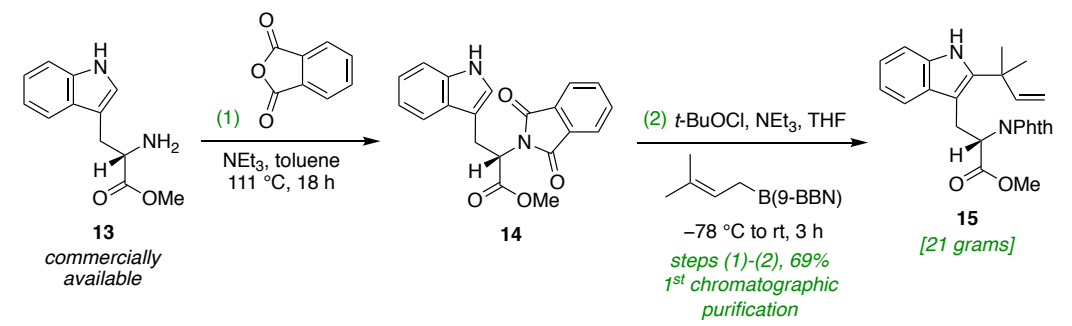

Step (2) - Based on a procedure reported by Danishefsky and co-workers. ${ }^{1}$ Reaction performed under anhydrous reaction conditions. A solution of crude compound 14 (25.0 g) and dry NEt 3 $(12.0 \mathrm{~mL}, 86.1 \mathrm{mmol})$ in THF $(250 \mathrm{~mL})$ was cooled to $-78{ }^{\circ} \mathrm{C}$, and the lights in the vicinity of the reaction were turned off. $t$ - $\mathrm{BuOCl}(9.7 \mathrm{~mL}, 86.1 \mathrm{mmol}$, see page 30 for preparation) was added dropwise and the reaction stirred for 30 minutes at $-78^{\circ} \mathrm{C}$. A solution of $B$-Prenyl-9-BBN (360 $\mathrm{mL}, 180 \mathrm{mmol}, 0.5 \mathrm{M}$ in THF, see pages $28-29$ for preparation) was added dropwise at $-78{ }^{\circ} \mathrm{C}$, over $50 \mathrm{~min}$. After stirring for $1 \mathrm{~h}$ at $-78^{\circ} \mathrm{C}$ the cooling bath was removed and the reaction slowly allowed to warm to room temperature. Reaction progress was monitored by TLC analysis, following the disappearance of starting material 14, $\mathrm{R}_{\mathrm{f}} 0.24(1: 1 \mathrm{EtOAc} /$ petroleum spirit) and appearance of an intermediate with $\mathrm{R}_{\mathrm{f}} 0.63$ (1:1 EtOAc/petroleum spirit). After stirring for $2 \mathrm{~h}$ saturated aq. $\mathrm{K}_{2} \mathrm{CO}_{3}(75 \mathrm{~mL})$ was added. The layers were separated and the aqueous layer extracted with EtOAc $(3 \times 125 \mathrm{~mL})$. The combined organic layers were dried $\left(\mathrm{MgSO}_{4}\right)$ and concentrated under reduced pressure. The crude product was filtered through silica with $\mathrm{CH}_{2} \mathrm{Cl}_{2}$, concentrated and purified by flash chromatography (1:9 to 1:3 EtOAc/petroleum spirit) to give compound $\mathbf{1 5}$ (21.1 g, $50.7 \mathrm{mmol}, 69 \%$ over 2 steps) as a pale-yellow foam. All spectroscopic data matched

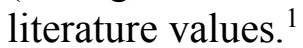

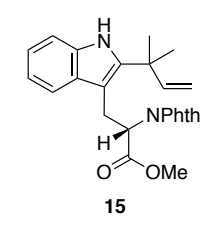

$\mathbf{R}_{\mathbf{f}} 0.55$ (1:1 EtOAc/petroleum spirit), 0.24 (1:4 EtOAc/petroleum spirit);

${ }^{1} \mathbf{H}$ NMR $\left(600 \mathrm{MHz}, \mathrm{CDCl}_{3}\right) \delta 7.86$ (br. s, $\left.1 \mathrm{H}\right), 7.69(\mathrm{dd}, J=5.5,3.0 \mathrm{~Hz}, 2 \mathrm{H}), 7.62(\mathrm{dd}, J=5.5$, $3.0 \mathrm{~Hz}, 2 \mathrm{H}), 7.28$ (app. dt, $J=7.9,0.9 \mathrm{~Hz}, 1 \mathrm{H}), 7.13$ (app. dt, $J=8.0,0.8 \mathrm{~Hz}, 1 \mathrm{H}), 6.90$ (ddd, $J=$ $8.1,7.0,1.1 \mathrm{~Hz}, 1 \mathrm{H}), 6.71$ (ddd, $J=8.0,7.0,1.0 \mathrm{~Hz}, 1 \mathrm{H}), 6.19(\mathrm{dd}, J=17.5,10.5 \mathrm{~Hz}, 1 \mathrm{H}), 5.23-$ $5.17(\mathrm{~m}, 2 \mathrm{H}), 5.15(\mathrm{dd}, J=10.5,1.0 \mathrm{~Hz}, 1 \mathrm{H}), 3.86(\mathrm{dd}, J=15.4,3.9 \mathrm{~Hz}, 1 \mathrm{H}), 3.78(\mathrm{~s}, 3 \mathrm{H}), 3.67$ (dd, $J=15.4,11.3 \mathrm{~Hz}, 1 \mathrm{H}), 1.58(\mathrm{~s}, 3 \mathrm{H}), 1.57$ (s, 3H) ppm;

${ }^{13}$ C NMR $\left(151 \mathrm{MHz}, \mathrm{CDCl}_{3}\right) \delta 169.7,167.8,146.0,140.3,134.0,134.0,132.0,129.9,123.3$, $121.3,119.3,117.9,112.3,110.3,106.4,53.6,52.9,39.3,27.8,27.7,24.6$ ppm;

$[\boldsymbol{\alpha}]_{D}^{23.7}-221.6^{\circ}\left(c 3.97, \mathrm{CHCl}_{3}\right)$, literature: $[\boldsymbol{\alpha}]_{D}^{25}-180.8^{\circ}\left(c 3.9, \mathrm{CHCl}_{3}\right) .^{1}$

\section{Reference:}

1. Schkeryantz, J. M., Woo, J. C. G., Siliphaivanh, P., Depew, K. M. \& Danishefsky, S. J. Total synthesis of gypsetin, deoxybrevianamide E, brevianamide E, and tryprostatin B: Novel constructions of 2,3-disubstituted indoles. J. Am. Chem. Soc. 121, 11964-11975 (1999). 
3.3 Experimental Procedure for Compound 16

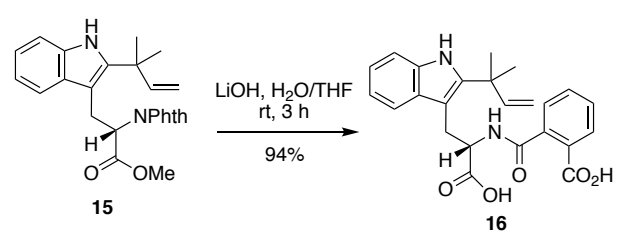

Compound 16 was prepared based on conditions reported by Ley and co-workers. ${ }^{1}$ To a solution of compound $15(67 \mathrm{mg}, 0.16 \mathrm{mmol})$ in $1: 1 \mathrm{THF} / \mathrm{H}_{2} \mathrm{O}(2 \mathrm{~mL})$ was added solid $\mathrm{LiOH}(19 \mathrm{mg}$, $0.79 \mathrm{mmol})$. The solution was stirred at $\mathrm{rt}$ for $3 \mathrm{~h}$ before being neutralised with $1 \mathrm{M}$ aq. $\mathrm{HCl}$ and the aqueous layer extracted with $\mathrm{Et}_{2} \mathrm{O}(3 \times 5 \mathrm{~mL})$. The combined organics were washed with $\mathrm{H}_{2} \mathrm{O}$ $(2 \times 2.5 \mathrm{~mL})$, brine $(2.5 \mathrm{~mL})$, dried $\left(\mathrm{Na}_{2} \mathrm{SO}_{4}\right)$ and concentrated under reduced pressure to give crude compound 16 (62 $\mathrm{mg}, 0.15 \mathrm{mmol}, 94 \%$ crude mass recovery) as a yellow foam, which was sufficiently pure for characterisation purposes.

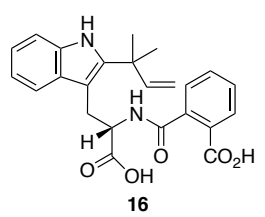

${ }^{1} \mathbf{H}$ NMR $\left(500 \mathrm{MHz}, \mathrm{CD}_{3} \mathrm{OD}\right) \delta 7.91(\mathrm{dd}, J=7.6,1.6 \mathrm{~Hz}, 1 \mathrm{H}), 7.60(\mathrm{~d}, J=7.9 \mathrm{~Hz}, 1 \mathrm{H}), 7.46$ (app. td, $J=7.6,1.6 \mathrm{~Hz}, 1 \mathrm{H}), 7.42$ (app. td, $J=7.5,1.6 \mathrm{~Hz}, 1 \mathrm{H}), 7.31(\mathrm{~d}, J=8.0 \mathrm{~Hz}, 1 \mathrm{H}), 7.04$ (ddd, $J$ $=8.1,6.9,1.2 \mathrm{~Hz}, 1 \mathrm{H}), 6.98-6.93(\mathrm{~m}, 1 \mathrm{H}), 6.89(\mathrm{dd}, J=7.4,1.5 \mathrm{~Hz}, 1 \mathrm{H}), 6.26(\mathrm{dd}, J=17.5,10.6$ $\mathrm{Hz}, 1 \mathrm{H}), 5.17$ (dd, $J=17.4,1.3 \mathrm{~Hz}, 1 \mathrm{H}), 5.11$ (dd, $J=10.5,1.2 \mathrm{~Hz}, 1 \mathrm{H}), 4.98$ (app. t, $J=7.8 \mathrm{~Hz}$, $1 \mathrm{H}), 3.51(\mathrm{dd}, J=14.5,7.7 \mathrm{~Hz}, 1 \mathrm{H}), 3.27$ (dd, $J=14.5,7.9 \mathrm{~Hz}, 1 \mathrm{H}), 1.59$ (s, 3H), 1.59 (s, 3H) ppm;

${ }^{13}$ C NMR (126 MHz, CD $\left.{ }_{3} \mathrm{OD}\right) \delta 175.3,172.2,169.2,147.6,142.1,139.4,136.4,132.9,131.2$, $131.1,130.5,130.4,129.0,121.7,119.6,119.3,112.1,111.6,106.6,55.6,40.4,28.9,28.5,28.4$ ppm;

IR (film, $\mathrm{cm}^{-1}$ ) 3000, 1771, 1694, 1597, 1525, 1462, 1389;

HRMS $\left(\mathrm{EI}^{+}\right) \mathrm{m} / \mathrm{z} 420.16655$ (calculated $[\mathrm{M}]^{+}:$420.16797);

$[\boldsymbol{\alpha}]_{D}^{23.4}-28.3^{\circ}(c 0.10, \mathrm{MeOH})$.

\section{Reference:}

1. Hewitt, P. R., Cleator, E. \& Ley, S. V. A concise total synthesis of (+)-okaramine C. Org. Biomol. Chem. 2, 2415-2417 (2004). 

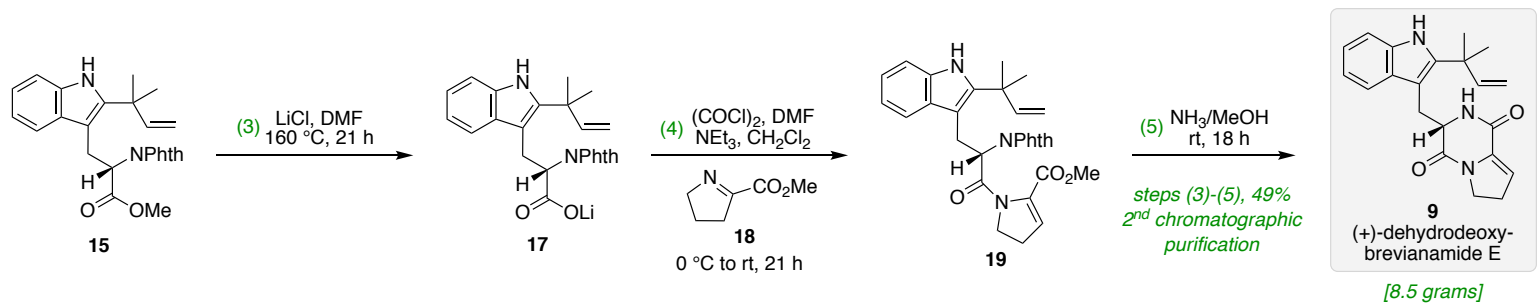

Step (3) - Inspired by the work of Hell, ${ }^{1,2}$ and Fisher. ${ }^{3}$ Compound 15 (24.6 g, 59.0 mmol) and $\mathrm{LiCl}(50.0 \mathrm{~g}, 1180 \mathrm{mmol})$ were suspended in DMF $(125 \mathrm{~mL})$ and the resulting mixture heated at $160{ }^{\circ} \mathrm{C}$ for $21 \mathrm{~h}$ under a flow of nitrogen. The thick brown suspension was diluted with $\mathrm{CH}_{2} \mathrm{Cl}_{2}$ $(300 \mathrm{~mL})$, sonicated for 30 minutes and the solid removed by filtration. The residual solid was broken up, suspended in $\mathrm{CH}_{2} \mathrm{Cl}_{2}(300 \mathrm{~mL})$, stirred for 30 minutes and filtered. The combined filtrates were concentrated under reduced pressure, then dried at $160{ }^{\circ} \mathrm{C}$ under reduced pressure. The crude product was re-dissolved in $\mathrm{CH}_{2} \mathrm{Cl}_{2}$, filtered and concentrated twice to remove any residual $\mathrm{LiCl}$ solid, before being dried at $160{ }^{\circ} \mathrm{C}$ under reduced pressure to give crude lithium carboxylate 17 (23.6 g), which was used without further purification in the next step.

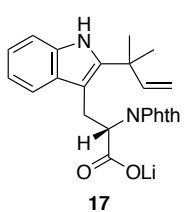

${ }^{1}$ H NMR (500 MHz, CD $\left.{ }_{3} \mathrm{OD}\right) \delta 7.62$ (app. s, 4H), 7.21 (app. dt, $\left.J=8.1,0.9 \mathrm{~Hz}, 1 \mathrm{H}\right), 7.11$ (app. t, $J=8.0,0.9 \mathrm{~Hz}, 1 \mathrm{H}), 6.75(\mathrm{ddd}, J=8.1,7.0,1.1 \mathrm{~Hz}, 1 \mathrm{H}), 6.53(\mathrm{ddd}, J=8.0,7.0,1.0 \mathrm{~Hz} 1 \mathrm{H}), 6.26$ $(\mathrm{dd}, J=17.4,10.6 \mathrm{~Hz}, 1 \mathrm{H}), 5.13(\mathrm{dd}, J=17.4,1.2 \mathrm{~Hz}, 1 \mathrm{H}), 5.04(\mathrm{dd}, J=10.6,1.2 \mathrm{~Hz}, 1 \mathrm{H}), 4.95$ (dd, $J=12.0,3.3 \mathrm{~Hz}, 1 \mathrm{H}), 3.84(\mathrm{dd}, J=15.3,3.3 \mathrm{~Hz}, 1 \mathrm{H}), 3.58(\mathrm{dd}, J=15.2,12.0 \mathrm{~Hz}, 1 \mathrm{H}), 1.59$ $(\mathrm{s}, 3 \mathrm{H}), 1.54(\mathrm{~s}, 3 \mathrm{H}) \mathrm{ppm}$;

${ }^{13}$ C NMR (126 MHz, CD $\left.{ }_{3} \mathrm{OD}\right) \delta 176.1,170.1,147.8,141.3,136.2,134.6,133.7,131.1,123.5$, $121.2,118.9,118.6,111.7,111.2,108.5,57.8,40.4,28.6,28.3,26.0$ ppm;

IR (film, $\mathrm{cm}^{-1}$ ) 2924, 2365, 1773, 1701, 1611, 1466, 1396, 1350;

HRMS $\left(\mathrm{ESI}^{-}\right) \mathrm{m} / z 401.1476$ (calculated $[\mathrm{M}-\mathrm{Li}]^{-}$401.1507);

$[\boldsymbol{\alpha}]_{D}^{22.9}-226.0^{\circ}(c 0.20, \mathrm{MeOH})$.

\section{References:}

1. Fölling, J., Belov, V., Kunetsky, R., Medda, R., Schönle, A., Egner, A., Eggeling, C., Bossi, M. \& Hell, S. W. Photochromic rhodamines provide nanoscopy with optical sectioning. Angew. Chem. Int. Ed. 46, 6266-6270 (2007).

2. Belov, V. N., Bossi, M. L., Fölling, J., Boyarskiy, V. P. \& Hell, S. W. Rhodamine spiroamides for multicolor single-molecule switching fluorescent nanoscopy. Chem. Eur. J. 15, 10762-10776 (2009).

3. Fisher, J. W. \& Trinkle, K. L. Iodide dealkylation of benzyl, PMB, PNB, and t-Butyl N-acyl amino acid esters via lithium ion coordination. Tetrahedron Lett. 35, 2505-2508 (1994). 

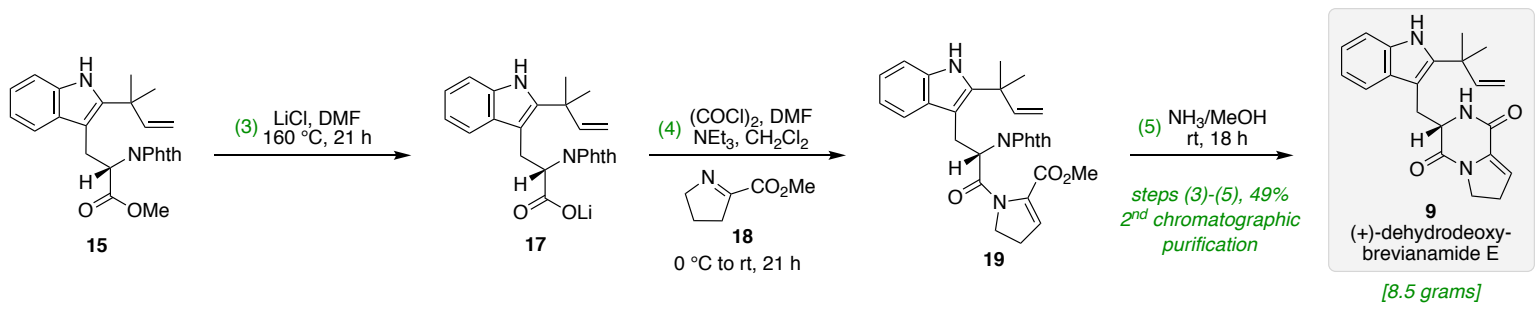

Step (4) - Inspired by the work of Schmalz, ${ }^{1}$ and Soai. ${ }^{2}$ Reaction performed under anhydrous reaction conditions. DMF $(1.90 \mathrm{~mL}, 24.6 \mathrm{mmol})$ was added dropwise to a solution of oxalyl chloride $(6.24 \mathrm{~mL}, 73.8 \mathrm{mmol})$ in $\mathrm{CH}_{2} \mathrm{Cl}_{2}(200 \mathrm{~mL})$ at $0{ }^{\circ} \mathrm{C}$. A suspension of crude compound 17 from Step (3) $(20.1 \mathrm{~g})$ in $\mathrm{CH}_{2} \mathrm{Cl}_{2}(200 \mathrm{~mL})$ was then added dropwise over $30 \mathrm{~min}$ at $0{ }^{\circ} \mathrm{C}$ and the residual 17 was washed into the reaction with $\mathrm{CH}_{2} \mathrm{Cl}_{2}(2 \times 50 \mathrm{~mL})$ and the resulting mixture was stirred at $0{ }^{\circ} \mathrm{C}$ for $30 \mathrm{~min}$. A solution of compound $18(5.84 \mathrm{~mL}, 49.2 \mathrm{mmol}$, see page 27 for preparation) and $\mathrm{NEt}_{3}(10.3 \mathrm{~mL}, 73.8 \mathrm{mmol})$ in $\mathrm{CH}_{2} \mathrm{Cl}_{2}$ was added dropwise over $20 \mathrm{~min}$ at $0{ }^{\circ} \mathrm{C}$. The cooling bath was then removed and the reaction stirred for $21 \mathrm{~h}$ at $\mathrm{rt}$. The reaction was quenched by the addition of $1 \mathrm{M}$ aq. $\mathrm{HCl}(80 \mathrm{~mL})$, the phases separated and the aqueous phase extracted with $\mathrm{CH}_{2} \mathrm{Cl}_{2}(3 \times 50 \mathrm{~mL})$. The combined organics were washed with saturated aq. $\mathrm{NaHCO}_{3}(80 \mathrm{~mL})$, and the aqueous layer then back extracted with $\mathrm{CH}_{2} \mathrm{Cl}_{2}(50 \mathrm{~mL})$. The combined organics were washed with brine $(120 \mathrm{~mL})$, dried $\left(\mathrm{Na}_{2} \mathrm{SO}_{4}\right)$ and concentrated under reduced pressure to give crude compound 19 (23.9 g) which was used without further purification in the next step. A small sample could be purified by flash chromatography (1:1 EtOAc/petroleum spirit) to give compound $\mathbf{1 9}$ as a lemon-yellow foam suitable for characterisation.

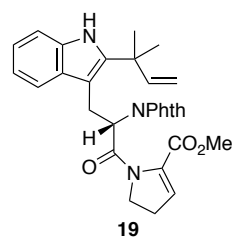

$\mathbf{R}_{\mathbf{f}} 0.28$ (1:1 EtOAc/petroleum spirit);

${ }^{1} \mathbf{H}$ NMR $\left(500 \mathrm{MHz}, \mathrm{CD}_{2} \mathrm{Cl}_{2}\right) \delta 7.95(\mathrm{~s}, 1 \mathrm{H}), 7.64$ (app. s, 4H), $7.17-7.13(\mathrm{~m}, 2 \mathrm{H}), 6.86$ (dd, $J=$ $8.2,7.1 \mathrm{~Hz}, 1 \mathrm{H}), 6.61$ (app. t, $J=7.4 \mathrm{~Hz}, 1 \mathrm{H}), 6.20$ (dd, $J=17.4,10.5 \mathrm{~Hz}, 1 \mathrm{H}), 5.87$ (dd, $J=3.5$, $2.5 \mathrm{~Hz}, 1 \mathrm{H}), 5.24(\mathrm{dd}, J=10.4,4.4 \mathrm{~Hz}, 1 \mathrm{H}), 5.20(\mathrm{dd}, J=17.5,1.1 \mathrm{~Hz}, 1 \mathrm{H}), 5.13(\mathrm{dd}, J=10.6$, $1.1 \mathrm{~Hz}, 1 \mathrm{H}), 3.94$ (br. s, 1H), 3.83 (dd, $J=15.4,4.4 \mathrm{~Hz}, 1 \mathrm{H}), 3.72$ (s, 3H), 3.62 (app. q, $J=10.5$ $\mathrm{Hz}, 1 \mathrm{H}), 3.46$ (dd, $J=15.4,10.4 \mathrm{~Hz}, 1 \mathrm{H}), 2.62$ (app. dtd, $J=17.6,10.0,2.5 \mathrm{~Hz}, 1 \mathrm{H}$ ), 2.39 (app. ddt, $J=17.5,10.6,3.4 \mathrm{~Hz}, 1 \mathrm{H}), 1.55(\mathrm{~s}, 3 \mathrm{H}), 1.55(\mathrm{~s}, 3 \mathrm{H})$;

${ }^{13} \mathrm{C}$ NMR $\left(151 \mathrm{MHz}, \mathrm{CD}_{2} \mathrm{Cl}_{2}\right) \delta 167.8,167.7,162.1,146.6,140.9,137.0,134.5,134.5,131.9$, 130.3, 123.6, 123.5, 121.5, 119.4, 118.1, 112.3, 110.6, 106.6, 54.2 (beneath solvent peak), 52.6, 50.4 (br.), 39.7, 29.6, 27.9, 27.9, 25.4;

IR (film, $\mathrm{cm}^{-1}$ ) 1775, 1713, 1655, 1639, 1611, 1437, 1381;

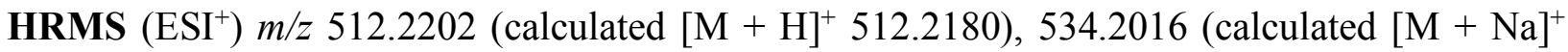
534.1999);

$[\boldsymbol{\alpha}]_{D}^{\mathbf{2 3 . 0}}-130.1^{\circ}(c 0.38, \mathrm{MeOH})$.

\section{References:}

1. Huy, P., Neudörfl, J.-M. \& Schmalz, H.-G. A practical synthesis of trans-3-substituted proline derivatives through 1,4-addition. Org. Lett. 13, 216-219 (2011).

2. Ookawa, A. \& Soai, K. Asymmetric synthesis of optically active threo- and erythropyrrolidinylbenzyl alcohol by the highly stereospecific arylation of $(S)$-proline and the subsequent highly diastereoselective reduction of the $\alpha$-amino ketone. J. Chem. Soc., Perkin Trans. $11465-$ 1465 (1987). 
3.6 Experimental Procedure for Compound 9
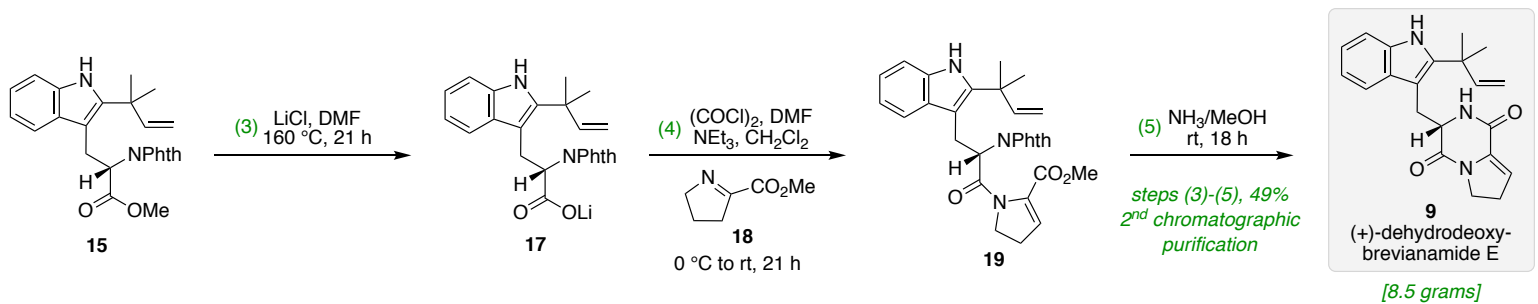

Step (5) - The crude product 19 (23.9 g) from Step (4) was dissolved in a solution of $\mathrm{NH}_{3}$ in $\mathrm{MeOH}(7 \mathrm{M}, 1200 \mathrm{~mL})$ and stirred at room temperature for $18 \mathrm{~h}$. The crude reaction mixture was concentrated under reduced pressure. Flash chromatography on neutralised silica $(7: 3$ to $8: 2$ EtOAc/petroleum spirit) gave (+)-dehydrodeoxybrevianamide E (9) (8.51 g, 24.4 mmol, 49\% over 3 steps) as a pale-yellow foam. All spectroscopic data matched literature values. ${ }^{1}$

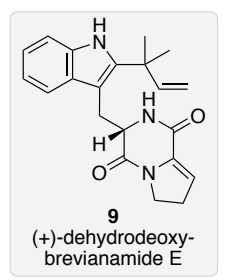

$\mathbf{R}_{\mathbf{f}} 0.19$ (8:2 EtOAc/petroleum spirit);

${ }^{1}$ H NMR $\left(500 \mathrm{MHz}, \mathrm{CDCl}_{3}\right) \delta 8.08$ (br. s, $\left.1 \mathrm{H}\right), 7.53$ (d, $\left.J=7.9 \mathrm{~Hz}, 1 \mathrm{H}\right), 7.32$ (app. dt, $J=8.1,0.9$ $\mathrm{Hz}, 1 \mathrm{H}), 7.17$ (ddd, $J=8.1,7.1,1.2 \mathrm{~Hz}, 1 \mathrm{H}), 7.11$ (ddd, $J=8.1,7.1,1.1 \mathrm{~Hz}, 1 \mathrm{H}), 6.14$ (app. $\mathrm{t}, J=$ $3.1 \mathrm{~Hz}, 1 \mathrm{H}), 6.12$ (dd, $J=17.2,10.4 \mathrm{~Hz}, 1 \mathrm{H}), 5.66$ (br. s, $1 \mathrm{H}), 5.20-5.15$ (m, 2H), 4.52 (ddd, $J=$ $11.3,3.5,1.8 \mathrm{~Hz}, 1 \mathrm{H}), 4.14-4.02(\mathrm{~m}, 2 \mathrm{H}), 3.73(\mathrm{dd}, J=14.7,3.7 \mathrm{~Hz}, 1 \mathrm{H}), 3.23$ (dd, $J=14.6$, $11.3 \mathrm{~Hz}, 1 \mathrm{H}), 2.78$ (app. td, $J=9.1,3.1 \mathrm{~Hz}, 2 \mathrm{H}), 1.55$ (s, 3H), 1.55 (s, 3H) ppm;

${ }^{13} \mathrm{C}$ NMR $\left(126 \mathrm{MHz}, \mathrm{CDCl}_{3}\right) \delta 162.7,156.6,145.8,141.8,134.4,133.2,128.9,122.3,120.2$, $118.9,118.3,112.6,110.9,104.6,57.6,45.7,39.2,30.9,28.1,28.0,27.9$ ppm;

IR (film, cm $\left.{ }^{-1}\right) 3331$ br., 2967, 2926, 1672, 1639, 1435;

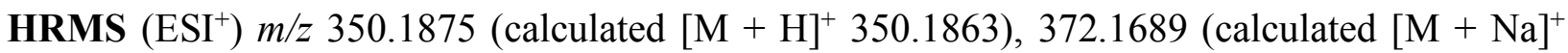
372.1682);

$[\boldsymbol{\alpha}]_{D}^{23.1}-33.2^{\circ}\left(c 1.30, \mathrm{CHCl}_{3}\right)$, literature: $[\boldsymbol{\alpha}]_{D}^{22}-38^{\circ}\left(c 1.3, \mathrm{CHCl}_{3}\right) .^{2}$

\section{References:}

1. Greshock, T. J. \& Williams, R. M. Improved biomimetic total synthesis of d,1-stephacidin A. Org. Lett. 9, 4255-4258 (2007).

2. Steyn, P. S. The structures of five diketopiperazines from Aspergillus ustus. Tetrahedron 29, 107-120 (1973). 


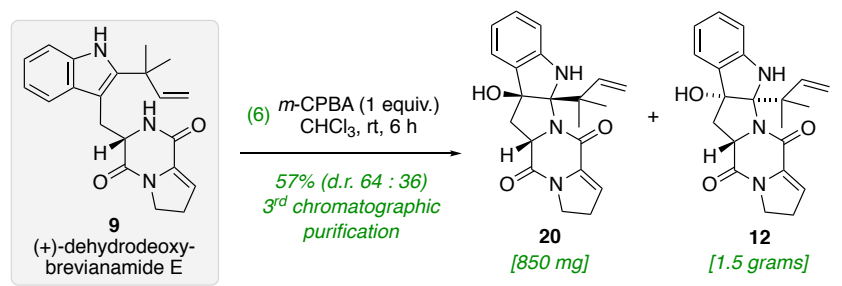

Step (6) - Inspired by the work of Kametani ${ }^{1}$ and Wolff. ${ }^{2}$ To a rapidly stirred solution of dehydrodeoxy-brevianamide $\mathrm{E}(9)(4.00 \mathrm{~g}, 11.5 \mathrm{mmol})$ in $\mathrm{CHCl}_{3}(80 \mathrm{~mL})$ at room temperature was added dropwise over $4 \mathrm{~h}$ a solution of $m$-CPBA in $\mathrm{CHCl}_{3}(0.29 \mathrm{M}, 40 \mathrm{~mL}, 11.7 \mathrm{mmol})$. ${ }^{*}$ The reaction was stirred for $2 \mathrm{~h}$ at room temperature and quenched by the addition of saturated aq. $\mathrm{Na}_{2} \mathrm{~S}_{2} \mathrm{O}_{3}(20 \mathrm{~mL})$ and saturated aq. $\mathrm{NaHCO}_{3}(20 \mathrm{~mL})$. The reaction mixture was diluted with EtOAc $(200 \mathrm{~mL})$ and $\mathrm{CHCl}_{3}(150 \mathrm{~mL})$ and the phases separated. The aqueous phase was back-extracted with $\mathrm{CHCl}_{3}(50 \mathrm{~mL})$ and the combined organics washed with saturated aq. $\mathrm{NaHCO}_{3}$ $(80 \mathrm{~mL})$, brine $(80 \mathrm{~mL})$, dried $\left(\mathrm{Na}_{2} \mathrm{SO}_{4}\right)$ and concentrated under reduced pressure. A diastereomeric ratio of 63:37 for 12:20 was determined by analysis of the ${ }^{1} \mathrm{H}$ NMR spectrum of this crude reaction product. Flash column chromatography $\left(1.5: 7: 3\right.$ petroleum spirit $/ \mathrm{CH}_{2} \mathrm{Cl}_{2} / i-\mathrm{Pr}_{2} \mathrm{O}$ to $7: 3 \mathrm{CH}_{2} \mathrm{Cl}_{2} / i-\mathrm{Pr}_{2} \mathrm{O}$ to EtOAc to $\left.i-\mathrm{PrOH}\right)$ of the crude reaction product gave dehydrobrevianamide E (12) (1.43 g, $3.91 \mathrm{mmol}, 34 \%)$ as a white foam, a fraction containing mixed dehydro-brevianamide E (12) and compound 20 (1.37 g, 18:82 12/20, $3.75 \mathrm{mmol}, 33 \%)$ as a cream foam, alongside recovered starting material dehydrodeoxy-brevianamide $\mathrm{E}$ (9) $(0.270 \mathrm{~g}, 0.774$ mmol, $7 \%$ ) as a cream foam. A second round of chromatography on the mixed 12/20 fraction gave additional pure dehydro-brevianamide E (12) $(0.108 \mathrm{~g}, 0.296 \mathrm{mmol}, 3 \%)$ as a cream glass, pure minor diastereomer $20(0.454 \mathrm{~g}, 1.24 \mathrm{mmol}, 11 \%)$ as a white foam, and a fraction containing mixed dehydro-brevianamide E (12) and compound 20 (0.638 g, 9:91 12/20, $1.75 \mathrm{mmol}, 15 \%)$. A third round of chromatography on this mixed $\mathbf{1 2 / 2 0}$ fraction gave additional pure minor diastereomer $20(0.405 \mathrm{~g}, 1.11 \mathrm{mmol}, 10 \%)$ as a white foam. Overall, purification by column chromatography gave dehydro-brevianamide E (12) (1.54 g, $4.21 \mathrm{mmol}, 37 \%)$, compound 20 (0.859 g, $2.35 \mathrm{mmol}$, $20 \%$ ), and recovered dehydrodeoxy-brevianamide E (9) (0.270 g, $0.774 \mathrm{mmol}, 7 \%)$.

* $m$-CPBA (69\% w/w by iodometric titration, $4.38 \mathrm{~g}, 17.5 \mathrm{mmol})$ was added to $\mathrm{CHCl}_{3}(60 \mathrm{~mL})$ and dried over $\mathrm{Na}_{2} \mathrm{SO}_{4}(27.5 \mathrm{~g})$ with stirring for 40 mins.

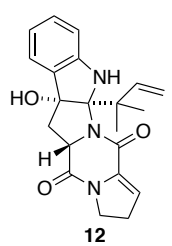

$\mathbf{R}_{\mathbf{f}} 0.15\left(7: 3 \mathrm{CH}_{2} \mathrm{Cl}_{2} / i-\mathrm{Pr}_{2} \mathrm{O}\right)$;

${ }^{1} \mathbf{H}$ NMR $\left(500 \mathrm{MHz}, \mathrm{CDCl}_{3}\right) \delta 7.24(\mathrm{dd}, J=7.5,1.2 \mathrm{~Hz}, 1 \mathrm{H}), 7.18(\mathrm{app} . \mathrm{td}, J=7.7,1.3 \mathrm{~Hz}, 1 \mathrm{H})$, $6.81(\mathrm{td}, J=7.4,1.0 \mathrm{~Hz}, 1 \mathrm{H}), 6.74$ (app. dt, $J=7.9,0.8 \mathrm{~Hz}, 1 \mathrm{H}), 6.40-6.34$ (m, 2H), 6.16 (app. t, $J=3.1 \mathrm{~Hz}, 1 \mathrm{H}), 5.15(\mathrm{dd}, J=17.7,1.4 \mathrm{~Hz}, 1 \mathrm{H}), 5.07(\mathrm{dd}, J=10.9,1.3 \mathrm{~Hz}, 1 \mathrm{H}), 4.06$ (ddd, $J=$ 12.0, 8.7, 7.3 Hz, 1H), $3.93-3.83(\mathrm{~m}, 1 \mathrm{H}), 3.81(\mathrm{dd}, J=11.5,7.3 \mathrm{~Hz}, 1 \mathrm{H}), 2.79-2.69(\mathrm{~m}, 4 \mathrm{H})$, 2.43 (br. s, 1H), 1.34 (s, 3H), 1.27 (s, 3H) ppm;

${ }^{13}$ C NMR (126 MHz, $\left.\mathrm{CDCl}_{3}\right) \delta 163.7,162.1,149.4,144.7,136.4,131.0,129.8,123.8,120.6$, $120.2,113.4,111.2,91.5,89.3,60.3,45.8,45.0,36.7,29.0,27.6,23.2$ ppm;

IR (film, cm cm $^{-1}$ 3362, 2967, 2926, 1670, 1634, 1609, 1485, 1468, 1437, 1393, 1360;

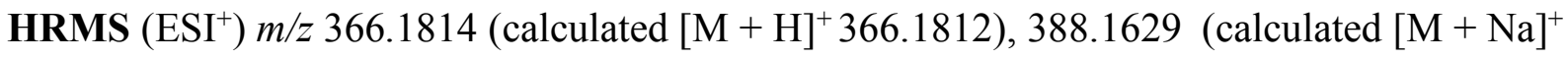
388.1632);

$[\boldsymbol{\alpha}]_{\boldsymbol{D}}^{\mathbf{2 0 . 9}}-208^{\circ}(c$ 0.23, EtOH). 


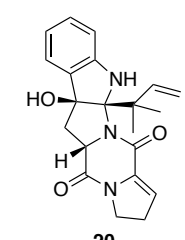

$\mathbf{R}_{\mathbf{f}} 0.09\left(7: 3 \mathrm{CH}_{2} \mathrm{Cl}_{2} / i-\mathrm{Pr}_{2} \mathrm{O}\right)$;

${ }^{1} \mathbf{H}$ NMR $\left(500 \mathrm{MHz}, \mathrm{CDCl}_{3}\right) \delta 7.22(\mathrm{~d}, J=7.5 \mathrm{~Hz}, 1 \mathrm{H}), 7.13$ (app. td, $\left.J=7.7,1.3 \mathrm{~Hz}, 1 \mathrm{H}\right), 6.76$ (app. td, $J=7.4,0.9 \mathrm{~Hz}, 1 \mathrm{H}$ ), 6.62 (app. dt, $J=7.9,0.8 \mathrm{~Hz}, 1 \mathrm{H}$ ), 6.47 (br. s, 1H), 6.34 (dd, $J=$ $17.7,10.8 \mathrm{~Hz}, 1 \mathrm{H}), 6.06$ (app. t, $J=3.0 \mathrm{~Hz}, 1 \mathrm{H}), 5.22$ (dd, $J=17.7,1.3 \mathrm{~Hz}, 1 \mathrm{H}), 5.10$ (dd, $J=$ $10.9,1.3 \mathrm{~Hz}, 1 \mathrm{H}$ ), 4.73 (app. t, $J=8.8 \mathrm{~Hz}, 1 \mathrm{H}), 4.00$ (ddd, $J=12.4,11.1,6.1 \mathrm{~Hz} 1 \mathrm{H}$ ), 3.82 (dddd, $J=12.3,11.4,8.3,0.8 \mathrm{~Hz}, 1 \mathrm{H}), 2.86(\mathrm{ddd}, J=13.6,8.6,0.7 \mathrm{~Hz}, 1 \mathrm{H}), 2.80-2.62(\mathrm{~m}, 3 \mathrm{H}), 2.57$ (br. s $1 \mathrm{H}), 1.45$ (s, 3H), 1.35 (s, 3H) ppm;

${ }^{13} \mathbf{C}$ NMR $\left(126 \mathrm{MHz}, \mathrm{CDCl}_{3}\right) \delta 161.9,156.2,147.0,144.8,134.7,131.5,130.4,123.8,119.5$, 119.3, 114.1, 109.9, 95.3, 89.6, 60.5, 46.6, 45.4, 43.4, 28.2, 27.2, 22.5 ppm;

IR (film, cm cm $^{-1}$ 3360, 2965, 2924, 2854, 1665, 1632, 1611, 1487, 1466, 1439, 1414, 1371;

HRMS $\left(\mathrm{ESI}^{+}\right) \mathrm{m} / z$ 366.1807 (calculated $[\mathrm{M}+\mathrm{H}]^{+} 366.1812$ );

$[\boldsymbol{\alpha}]_{D}^{22.3}+86.3^{\circ}(c 0.19, \mathrm{EtOH})$.

\section{References:}

1. Kametani, T., Kanaya, N. \& Ihara, M. Asymmetric total synthesis of brevianamide E. J. Am. Chem. Soc. 1980, 102 (11), 3974-3975.

2. Adam, W., Bosio, S. G. \& Wolff, B. T. Chiral-Auxiliary-Controlled Diastereoselectivity in the Epoxidation of Enecarbamates with DMD and mCPBA. Org. Lett. 2003, 5 (6), 819-822. 

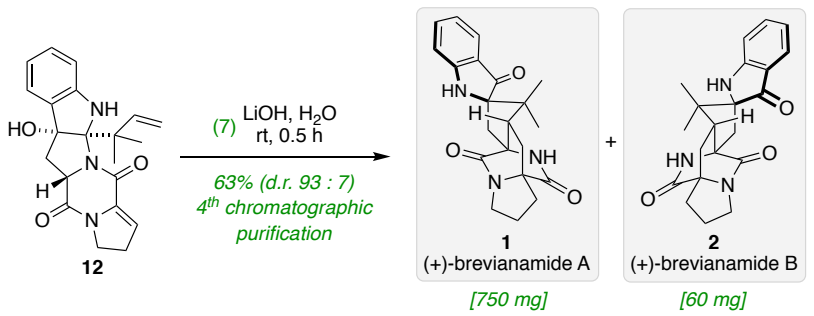

Step (7) - The synthesis of brevianamide A (1) was carried out under aerobic conditions, inspired by the work of Kishi ${ }^{1}$ and Williams. ${ }^{2}$ Effort was made to limit the exposure of the material to light once the reaction was complete, to prevent photolysis of brevianamide A (1) to brevianamide C and D. ${ }^{3}$ To a solution of dehydro-brevianamide E (12) $(1.30 \mathrm{~g}, 3.56 \mathrm{mmol})$ was added a $1 \mathrm{M}$ solution of aq. $\mathrm{LiOH}(450 \mathrm{~mL})$ and the reaction stirred rapidly, with vigorous manual shaking every 2-3 minutes to facilitate gradual dissolution of the starting material. After 30 minutes the reaction mixture was extracted with $\mathrm{CH}_{2} \mathrm{Cl}_{2}(2 \times 450 \mathrm{~mL}, 2 \times 200 \mathrm{~mL})$, and the combined organics dried $\left(\mathrm{Na}_{2} \mathrm{SO}_{4}\right)$. Flash column chromatography $\left(2: 8\right.$ to $3: 7$ to $1: 1 \mathrm{THF} / \mathrm{CHCl}_{3}$ to $25: 25: 1$ $\left.\mathrm{THF} / \mathrm{CHCl}_{3} / i-\mathrm{PrOH}\right)$ gave (+)-brevianamide A (1) $\left(905 \mathrm{mg}, 16.2 \% \mathrm{CHCl}_{3} \mathrm{w} / \mathrm{w}, 2.07 \mathrm{mmol}\right.$, $58 \%)^{*}$ as a yellow amorphous solid and (+)-brevianamide B (2) $(59.8 \mathrm{mg}, 0.164 \mathrm{mmol}, 5 \%)$ as a yellow crystalline solid. A sample of (+)-brevianamide A (1) was crystallised from $\mathrm{CHCl}_{3}$ by slow vapour diffusion with $\mathrm{Et}_{2} \mathrm{O}$. See pages 58-68 for X-ray crystal structure of brevianamide $\mathrm{A} \cdot \mathrm{CHCl}_{3}$.

*In Birch's original isolation paper brevianamide A 'crystallized from $\mathrm{CHCl}_{3}$ in needles containing one molecule of solvent of crystallisation'. ${ }^{4}$ The reported crystal structure for 5-bromo-brevianamide A also showed co-crystallisation with one molecule of acetone. ${ }^{5}$ Consistent with these observations, we found that a certain portion of solvent could not be removed from brevianamide A under high vacuum. Proteo-solvent was replaced with deutero-solvent to record NMR spectra for characterisation.

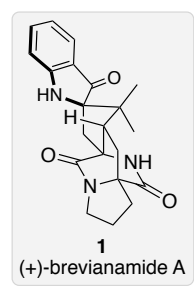

Spectroscopic data matched literature values reported by Zhu Weiming and co-workers. ${ }^{6}$ Subtle differences in ${ }^{1} \mathrm{H}$ NMR data are attributed to concentration effects in $\mathrm{CDCl}_{3}$ (see page 42).

$\mathbf{R}_{\mathbf{f}} 0.21\left(3: 7 \mathrm{THF} / \mathrm{CHCl}_{3}\right)$;

MP $173-179{ }^{\circ} \mathrm{C}$, literature: $175-180{ }^{\circ} \mathrm{C}$ ' with loss of solvent' ${ }^{4}$

${ }^{1} \mathbf{H}$ NMR $\left(500 \mathrm{MHz}, \mathrm{CDCl}_{3}\right) \delta 7.57(\mathrm{dd}, J=7.7,1.2 \mathrm{~Hz}, 1 \mathrm{H}), 7.44(\mathrm{ddd}, J=8.3,7.1,1.4 \mathrm{~Hz}, 1 \mathrm{H})$, $6.84-6.78(\mathrm{~m}, 2 \mathrm{H}), 6.62$ (br. s, $1 \mathrm{H}), 4.98$ (br. s, $1 \mathrm{H}), 3.52-3.41(\mathrm{~m}, 2 \mathrm{H}), 2.81-2.74(\mathrm{~m}, 2 \mathrm{H})$, $2.40(\mathrm{ddd}, J=9.8,7.3,1.1 \mathrm{~Hz}, 1 \mathrm{H}), 2.35$ (d, $J=15.6 \mathrm{~Hz}, 1 \mathrm{H}), 2.04$ (app. pd, $J=6.8,1.6 \mathrm{~Hz}, 2 \mathrm{H})$, $1.96-1.81(\mathrm{~m}, 3 \mathrm{H}), 1.12(\mathrm{~s}, 3 \mathrm{H}), 0.93(\mathrm{~s}, 3 \mathrm{H}) \mathrm{ppm}$;

${ }^{13}$ C NMR $\left(126 \mathrm{MHz}, \mathrm{CDCl}_{3}\right) \delta 202.2,172.4,169.8,160.2,137.8,124.8,121.2,119.3,112.1,78.9$, 69.5, 67.8, 55.7, 48.4, 44.1, 37.4, 29.2, 29.0, 25.1, 24.1, 19.9 ppm;

${ }^{1}$ H NMR $\left(500 \mathrm{MHz},\left(\mathrm{CD}_{3}\right)_{2} \mathrm{SO}\right) \delta 8.65$ (br. s, $\left.1 \mathrm{H}\right), 7.84$ (br. s, $\left.1 \mathrm{H}\right), 7.41$ (ddd, $J=8.3,7.0,1.4 \mathrm{~Hz}$, $1 \mathrm{H}), 7.37(\mathrm{~d}, J=7.7 \mathrm{~Hz}, 1 \mathrm{H}), 6.81(\mathrm{dd}, J=8.2,0.8 \mathrm{~Hz}, 1 \mathrm{H}), 6.64(\mathrm{ddd}, J=7.8,7.0,0.8 \mathrm{~Hz}, 1 \mathrm{H})$, $3.34-3.23(\mathrm{~m}, 2 \mathrm{H}), 2.55$ (ddd, $J=10.1,7.5,1.1 \mathrm{~Hz}, 1 \mathrm{H}), 2.49-2.44(\mathrm{~m}, 2 \mathrm{H}), 2.39(\mathrm{~d}, J=15.1$ $\mathrm{Hz}, 1 \mathrm{H}), 2.01-1.91(\mathrm{~m}, 1 \mathrm{H}), 1.90-1.77(\mathrm{~m}, 3 \mathrm{H}), 1.68(\mathrm{dd}, J=13.1,7.6 \mathrm{~Hz}, 1 \mathrm{H}), 0.98(\mathrm{~s}, 3 \mathrm{H})$, 0.68 (s, 3H) ppm; 
${ }^{13}$ C NMR (126 MHz, $\left.\left(\mathrm{CD}_{3}\right)_{2} \mathrm{SO}\right) \delta$ 199.9, 172.1, 169.5, 160.5, 137.2, 123.6, 119.4, 116.7, 111.1, 79.0, 68.5, 66.4, 53.1, 48.3, 43.2, 39.5, 28.4, 27.2, 24.6, 21.2, 17.7 ppm;

IR (film, cm ${ }^{-1}$ ) 3314, 3229, 2938, 2876, 1667, 1618, 1491, 1466, 1395;

HRMS $\left(\mathrm{ESI}^{+}\right) \mathrm{m} / z$ 366.1818 (calculated $[\mathrm{M}+\mathrm{H}]^{+} 366.1812$ );

$[\boldsymbol{\alpha}]_{D}^{\mathbf{2 3 . 5}}+316^{\circ}(c$ 0.12, EtOH $)$, literature: $[\boldsymbol{\alpha}]_{\boldsymbol{D}}^{\mathbf{2 5}}+413^{\circ}(\mathrm{EtOH}) \dot{4}^{4}$

e.r. 93:7, after crystallisation 99:1 (Chiralpak IA, 1:1 $i$-PrOH/hexane, $1 \mathrm{~mL} \mathrm{~min}^{-1}, \lambda 254 \mathrm{~nm}$ ) $t_{\text {Rminor }}$ $=5.41 \mathrm{~min}, \mathrm{t}_{\mathrm{Rmajor}}=6.72 \mathrm{~min}$. See Pages 54-55 for chiral HPLC traces.

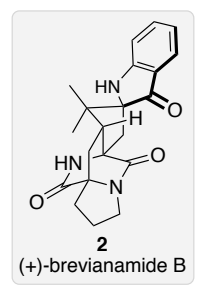

Brevianamide $\mathrm{B}$ (2) was only sparingly soluble in $\mathrm{CDCl}_{3}$, consistent with the observation in Birch's original isolation paper that brevianamide B 'was insoluble in most solvents except hot DMSO and $\mathrm{CF}_{3} \mathrm{CO}_{2} \mathrm{H}$, the latter causing decomposition'. ${ }^{3}{ }^{1} \mathrm{H}$ and ${ }^{13} \mathrm{C}$ NMR spectra could be recorded using a saturated sample in $\mathrm{CDCl}_{3}$, to enable comparison to literature data, ${ }^{7,8}$ however 2D NMR spectra were unobtainable. High quality 1D and 2D NMR spectra were instead recorded in $\left(\mathrm{CD}_{3}\right)_{2} \mathrm{SO}$.

$\mathbf{R}_{\mathbf{f}} 0.08\left(3: 7 \mathrm{THF} / \mathrm{CHCl}_{3}\right)$;

MP 286-290 ${ }^{\circ} \mathrm{C}$ (decomposition), literature: $324-328^{\circ} \mathrm{C}$ (decomposition); ${ }^{3}$

${ }^{1} \mathbf{H}$ NMR (500 MHz, $\mathrm{CDCl}_{3}$ ) $\delta 7.56$ (app. ddt, $\left.J=7.6,1.3,0.7 \mathrm{~Hz}, 1 \mathrm{H}\right), 7.43$ (ddd, $J=8.4,7.1,1.4$ $\mathrm{Hz}, 1 \mathrm{H}), 6.84-6.78$ (m, 2H), 5.78 (br. s, 1H), 4.73 (br. s, 1H), $3.50-3.44$ (m, 2H), 3.31 (ddd, $J$ $=10.3,7.4,1.3 \mathrm{~Hz}, 1 \mathrm{H}), 3.27(\mathrm{~d}, J=15.6 \mathrm{~Hz}, 1 \mathrm{H}), 2.74(\mathrm{ddd}, J=13.1,7.0,6.1 \mathrm{~Hz}, 1 \mathrm{H}), 2.06-$ $1.93(\mathrm{~m}, 3 \mathrm{H}), 1.90-1.77(\mathrm{~m}, 2 \mathrm{H}), 1.70(\mathrm{~d}, J=15.7,1 \mathrm{H}), 1.14(\mathrm{~s}, 3 \mathrm{H}), 0.83(\mathrm{~s}, 3 \mathrm{H}) \mathrm{ppm}$;

${ }^{13}$ C NMR $\left(126 \mathrm{MHz}, \mathrm{CDCl}_{3}\right) \delta 203.9,173.4,169.2,160.3,137.5,125.2,120.1,119.2,111.5,77.7$, 68.9, 66.5, 49.7, 46.6, 44.0, 36.8, 29.3, 28.6, 25.1, 22.6, 20.5 ppm;

${ }^{1} \mathbf{H}$ NMR $\left(500 \mathrm{MHz},\left(\mathrm{CD}_{3}\right)_{2} \mathrm{SO}\right) \delta 8.64$ (br. s, $\left.1 \mathrm{H}\right), 7.41$ (ddd, $\left.J=8.3,7.1,1.4 \mathrm{~Hz}, 1 \mathrm{H}\right), 7.33(\mathrm{~d}, J$ $=7.7 \mathrm{~Hz}, 1 \mathrm{H}), 7.27$ (br. s, $1 \mathrm{H}), 6.89$ (app. dt, $J=8.3,0.9 \mathrm{~Hz}, 1 \mathrm{H}), 6.64$ (ddd, $J=7.8,7.0,0.9 \mathrm{~Hz}$, 1H), $3.35-3.24$ (m, 2H), 3.01 (ddd, $J=10.3,7.5,1.1 \mathrm{~Hz}, 1 \mathrm{H}), 2.67$ (d, $J=15.1 \mathrm{~Hz}, 1 \mathrm{H}), 2.46$ $(\mathrm{dd}, J=11.9,6.1 \mathrm{~Hz}, 1 \mathrm{H}), 2.02-1.90(\mathrm{~m}, 3 \mathrm{H}), 1.85-1.74(\mathrm{~m}, 2 \mathrm{H}), 1.63(\mathrm{dd}, J=13.0,7.5 \mathrm{~Hz}$, $1 \mathrm{H}), 1.05(\mathrm{~s}, 3 \mathrm{H}), 0.63(\mathrm{~s}, 3 \mathrm{H}) \mathrm{ppm}$;

${ }^{13}$ C NMR (126 MHz, $\left.\left(\mathrm{CD}_{3}\right)_{2} \mathrm{SO}\right) \delta$ 204.7, 172.6, 169.2, 161.3, 137.2, 123.8, 118.1, 116.9, 111.4, 77.5, 68.1, 65.2, 48.8, 46.0, 43.3, 33.4, 28.4, 27.5, 24.4, 21.9, $19.7 \mathrm{ppm}$;

IR (film, cm ${ }^{-1}$ ) 3348, 3231, 2949, 2878, 1705, 1664, 1653, 1616, 1587, 1495, 1468;

HRMS $\left(\mathrm{ESI}^{+}\right) \mathrm{m} / z$ 366.1796 (calculated $[\mathrm{M}+\mathrm{H}]^{+} 366.1812$ );

$[\boldsymbol{\alpha}]_{\boldsymbol{D}}^{\mathbf{2 4 . 7}}+136^{\circ}\left(c 0.23,2.5 \% \mathrm{HCO}_{2} \mathrm{H}\right.$ in $\left.\mathrm{CH}_{2} \mathrm{Cl}_{2}\right)$, literature (enantiomer): $[\boldsymbol{\alpha}]_{\boldsymbol{D}}^{\mathbf{2 5}}-147^{\circ}(c 0.23,2.5 \%$ $\mathrm{HCO}_{2} \mathrm{H}$ in $\left.\mathrm{CH}_{2} \mathrm{Cl}_{2}\right),{ }^{8}[\boldsymbol{\alpha}]_{\boldsymbol{D}}^{25}-124^{\circ}\left(c 0.81,2.5 \% \mathrm{HCO}_{2} \mathrm{H}\right.$ in $\left.\mathrm{CH}_{2} \mathrm{Cl}_{2}\right){ }^{7}$

e.r. $93: 7$ (Chiralpak IA, 1:3 EtOH/hexane, $\left.1 \mathrm{~mL} \mathrm{m^{-1 }}, \lambda 254 \mathrm{~nm}\right) t_{\mathrm{Rminor}}=13.99 \mathrm{~min}, \mathrm{t}_{\mathrm{Rmajor}}=$ 26.10 min. See Pages 56-57 for chiral HPLC traces.

\section{References:}

1. Hutchison, A. J. \& Kishi, Y. Stereospecific total synthesis of dl-austamide. J. Am. Chem. Soc. 101, 6786-6788 (1979).

2. Greshock, T. J. \& Williams, R. M. Improved biomimetic total synthesis of d,l- stephacidin A. Org. Lett. 9, 4255-4258 (2007). 
3. Birch, A. J. \& Russell, R. A. Studies in relation to biosynthesis-XLIV: Structural elucidations of brevianamides-B, -C, -D and -F. Tetrahedron 28, 2999-3008 (1972).

4. Birch, A. J. \& Wright, J. J. Studies in relation to biosynthesis-XLII: The structural elucidation and some aspects of the biosynthesis of the brevianamides-A and -E. Tetrahedron 26, 2329-2344 (1970).

5. Coetzer, J. The structure and absolute configuration of 5-bromobrevianamide A. Acta Crystallographica Section B 30, 2254-2256 (1974).

6. Lingling, C., Tonghan, Z., Guoliang, Z., Yunlong, L., Cong, W., Pawinee, P., Arthit, C. \& Weiming, Z. Bioactive Natural Products from the Marine-Derived Penicillium brevicompactum OUCMDZ-4920. Chin. J. Org. Chem. 37, 2752-2762 (2017).

7. Williams, R. M., Glinka, T., Kwast, E., Coffman, H. \& Stille, J. K. Asymmetric, stereocontrolled total synthesis of (-)-brevianamide B. J. Am. Chem. Soc. 112, 808-821 (1990).

8. Frebault, F. C. \& Simpkins, N. S. A cationic cyclisation route to prenylated indole alkaloids: synthesis of malbrancheamide B and brevianamide B, and progress towards stephacidin A. Tetrahedron 66, 6585-6596 (2010). 

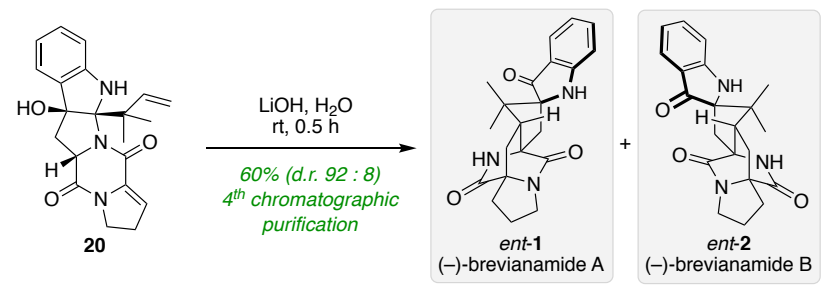

To a solution of compound $20(234 \mathrm{mg}, 0.640 \mathrm{mmol})$ was added a $1 \mathrm{M}$ solution of aq. LiOH $(80 \mathrm{~mL})$ and the reaction stirred rapidly, with vigorous manual shaking every $2-3$ minutes to facilitate gradual dissolution of the starting material. After 30 minutes the reaction mixture was extracted with $\mathrm{CH}_{2} \mathrm{Cl}_{2}(4 \times 100 \mathrm{~mL})$, and the combined organics dried $\left(\mathrm{Na}_{2} \mathrm{SO}_{4}\right)$. Flash column chromatography (2:8 to $3: 7$ to $1: 1 \mathrm{THF} / \mathrm{CHCl}_{3}$ to $25: 25: 1 \mathrm{THF} / \mathrm{CHCl}_{3} / i$-PrOH) gave (-)brevianamide A (ent-1) $\left(155 \mathrm{mg}, 17.5 \% \mathrm{CHCl}_{3} \mathrm{w} / \mathrm{w}, 0.350 \mathrm{mmol}, 55 \%\right) *$ as a yellow amorphous solid and (-)-brevianamide B (ent-2) (12 $\mathrm{mg}, 0.032 \mathrm{mmol}, 5 \%)$ as a yellow crystalline solid.

*In Birch's original isolation paper brevianamide A 'crystallized from $\mathrm{CHCl}_{3}$ in needles containing one molecule of solvent of crystallisation'. ${ }^{1}$ The reported crystal structure for 5-bromo-brevianamide A also showed co-crystallisation with one molecule of acetone. ${ }^{2}$ Consistent with these observations, we found that a certain portion of solvent could not be removed from brevianamide A under high vacuum. Proteo-solvent was replaced with deutero-solvent to record NMR spectra for characterisation.

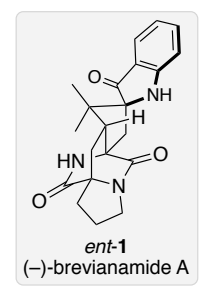

$[\boldsymbol{\alpha}]_{\boldsymbol{D}}^{\mathbf{2 4 . 7}}-281^{\circ}(c \mathrm{0} 0.12, \mathrm{EtOH})$, literature (enantiomer): $[\boldsymbol{\alpha}]_{\boldsymbol{D}}^{\mathbf{2 5}}+413^{\circ}(\mathrm{EtOH}) \dot{1}^{1}$

e.r. 95:5 (Chiralpak IA, 1:1 $i$-PrOH/hexane, $\left.1 \mathrm{~mL} \mathrm{~min}^{-1}, \lambda 254 \mathrm{~nm}\right) t_{\text {Rmajor }}=5.38 \mathrm{~min}$, $\mathrm{t}_{\mathrm{Rminor}}=$ 6.74 min. See Pages 54-55 for chiral HPLC traces.

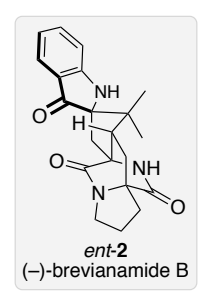

$[\boldsymbol{\alpha}]_{D}^{25.1}-119^{\circ}\left(c 0.23,2.5 \% \mathrm{HCO}_{2} \mathrm{H}\right.$ in $\left.\mathrm{CH}_{2} \mathrm{Cl}_{2}\right)$, literature: $[\boldsymbol{\alpha}]_{D}^{25}-147^{\circ}\left(c 0.23,2.5 \% \mathrm{HCO}_{2} \mathrm{H}\right.$ in $\left.\mathrm{CH}_{2} \mathrm{Cl}_{2}\right),{ }^{3}[\boldsymbol{\alpha}]_{\boldsymbol{D}}^{25}-124^{\circ}$ (c $0.81,2.5 \% \mathrm{HCO}_{2} \mathrm{H}$ in $\left.\mathrm{CH}_{2} \mathrm{Cl}_{2}\right) ;{ }^{4}$

e.r. $92: 8$ (Chiralpak IA, 1:3 EtOH/hexane, $\left.1 \mathrm{~mL} \mathrm{~min}^{-1}, \lambda 254 \mathrm{~nm}\right) \mathrm{t}_{\mathrm{Rmajor}}=13.86 \mathrm{~min}, \mathrm{t}_{\mathrm{Rminor}}=$ 26.14 min. See Pages 56-57 for chiral HPLC traces.

\section{References:}

1. Birch, A. J., \& Wright, J. J. Studies in relation to biosynthesis-XLII: The structural elucidation and some aspects of the biosynthesis of the brevianamides-A and -E. Tetrahedron 1970, 26 (10), 2329-2344.

2. Coetzer, J. The structure and absolute configuration of 5-bromobrevianamide A. Acta Crystallographica Section B 30, 2254-2256 (1974). 
3. Frebault, F. C. \& Simpkins, N. S. A cationic cyclisation route to prenylated indole alkaloids: synthesis of malbrancheamide $\mathrm{B}$ and brevianamide $\mathrm{B}$, and progress towards stephacidin A. Tetrahedron 66, 6585-6596 (2010).

4. Williams, R. M., Glinka, T., Kwast, E., Coffman, H. \& Stille, J. K. Asymmetric, stereocontrolled total synthesis of (-)-brevianamide B. J. Am. Chem. Soc. 112, 808-821 (1990). 
3.10 Experimental Procedure for Compound 18

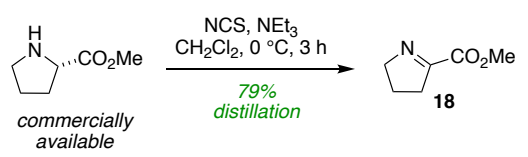

Compound 18 was prepared under anhydrous conditions, based on a procedure reported by Schmalz and co-workers. ${ }^{1}$ To a rapidly stirred suspension of L-proline methyl ester hydrochloride $(25.0 \mathrm{~g}, 151 \mathrm{mmol})$ and $\mathrm{NEt}_{3}(48.3 \mathrm{~mL}, 347 \mathrm{mmol})$ in $\mathrm{CH}_{2} \mathrm{Cl}_{2}(450 \mathrm{~mL})$ at $0{ }^{\circ} \mathrm{C}$ was added slowly, portion-wise, solid NCS $(22.2 \mathrm{~g}, 166 \mathrm{mmol})$. The mixture was stirred at room temperature for $3 \mathrm{~h}$ and then diluted with $\mathrm{Et}_{2} \mathrm{O}(350 \mathrm{~mL})$. A mixture of saturated aq. $\mathrm{NH}_{4} \mathrm{Cl}(150 \mathrm{~mL})$ and water $(150$ $\mathrm{mL}$ ) was added, the organic phase was separated and the aqueous phase was back-extracted with $\mathrm{CH}_{2} \mathrm{Cl}_{2}(2 \times 150 \mathrm{~mL})$. The combined organics were washed with a mixture of saturated aq. $\mathrm{NH}_{4} \mathrm{Cl}$ $(100 \mathrm{~mL})$ and water $(100 \mathrm{~mL})$, saturated aq. $\mathrm{NaHCO}_{3}(150 \mathrm{~mL})$, brine $(150 \mathrm{~mL})$, dried $\left(\mathrm{Na}_{2} \mathrm{SO}_{4}\right)$ and concentrated under reduced pressure. Vacuum distillation under reduced pressure ( 5 mbar) gave imine 18 (15.1 g, $119 \mathrm{mmol}, 79 \%)$ as a pale-yellow liquid. All spectroscopic data matched literature values. ${ }^{2}$<smiles>CC(=O)N1CCCC1</smiles>

BP $60-64{ }^{\circ} \mathrm{C}(5 \mathrm{mbar})$;

${ }^{1} \mathbf{H}$ NMR $\left(500 \mathrm{MHz}, \mathrm{CDCl}_{3}\right) \delta 4.04$ (app. tt, $\left.J=7.6,2.6 \mathrm{~Hz}, 2 \mathrm{H}\right), 3.80$ (s, 3H), 2.76 (app. ddt, $J=$ 8.5, 7.6, $2.6 \mathrm{~Hz}, 2 \mathrm{H}), 1.96-1.89(\mathrm{~m}, 2 \mathrm{H}) \mathrm{ppm}$;

${ }^{13} \mathbf{C}$ NMR $\left(126 \mathrm{MHz}, \mathrm{CDCl}_{3}\right) \delta 168.2,163.2,62.5,52.5,35.3,22.1 \mathrm{ppm}$.

\section{References:}

1. Huy, P., Neudörfl, J.-M. \& Schmalz, H.-G. A practical synthesis of trans-3- substituted proline derivatives through 1,4-addition. Org. Lett. 13, 216-219 (2011).

2. Sezen, B. \& Sames, D. Oxidative C-Arylation of Free (NH)-Heterocycles via Direct $\left(\mathrm{sp}^{3}\right) \mathrm{C}-\mathrm{H}$ Bond Functionalization. J. Am. Chem. Soc. 126, 13244-13246 (2004). 
3.11 Experimental Procedure for $B$-Prenyl-9-BBN

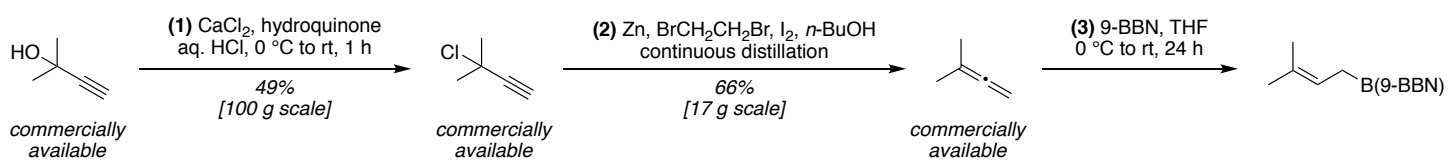

1,1-Dimethylallene is a commercially available reagent, but was prepared on large scale based on a procedure reported by Pfeffer and co-workers. ${ }^{1}$

Step (1) - Anhydrous $\mathrm{CaCl}_{2}(229 \mathrm{~g}, 2.06 \mathrm{~mol})$ was added in portions to a stirred mixture of concentrated hydrochloric acid $(800 \mathrm{~mL})$, 2-methyl-3-butyn-2-ol $(200 \mathrm{~mL}, 2.06 \mathrm{~mol})$ and hydroquinone $(1.82 \mathrm{~g}, 16.5 \mathrm{mmol})$ at $0{ }^{\circ} \mathrm{C}$ over 7 minutes and the reaction was stirred for $1 \mathrm{~h}$ at room temperature. The layers were seperated and solid $\mathrm{K}_{2} \mathrm{CO}_{3}$ added cautiously to the organic phase until effervescence ceased. The organic phase was then distilled under reduced pressure, collected up to $50{ }^{\circ} \mathrm{C}$ at 147 mbar. The crude distilate was redistilled under reduced pressure (147 mbar) to give 3-chloro-3-methyl-1-butyne (103 g, $1.00 \mathrm{~mol}, 49 \%)$ as a colourless oil. All spectroscopic data matched a comercially available sample.<smiles>[SiH3]C12CC1C2</smiles>

BP $32-36{ }^{\circ} \mathrm{C}(147$ mbar $)$;

${ }^{1} \mathbf{H}$ NMR $\left(500 \mathrm{MHz}, \mathrm{CDCl}_{3}\right) \delta 2.62(\mathrm{~s}, 1 \mathrm{H}), 1.87$ (s, 6H) ppm;

${ }^{13} \mathbf{C}$ NMR $\left(101 \mathrm{MHz}, \mathrm{CDCl}_{3}\right) \delta 86.7,72.0,57.1,34.7 \mathrm{ppm}$.

Step (2) - Zinc powder $(52.0 \mathrm{~g}, 760 \mathrm{mmol})$ was washed with $3 \%$ aq. $\mathrm{HCl}(4 \times 40 \mathrm{~mL})$, $2 \%$ aq. $\mathrm{CuSO}_{4}(2 \times 60 \mathrm{~mL})$, ethanol $(2 \times 60 \mathrm{~mL})$ and $n$-butanol $(2 \times 60 \mathrm{~mL})$. The treated zinc was suspended in $n$-butanol $(110 \mathrm{~mL})$ in a 3 neck RBF with a dropping funnel, magnetic stirrer bar and a distillation head fitted with a $30 \mathrm{~cm}$ Vigreux column. 3-Chloro-3-methyl-1-butyne $(44.4 \mathrm{~mL}$, $390 \mathrm{mmol})$ was placed in the dropping funnel along with 1,2-dibromoethane $(2.50 \mathrm{~mL}$, $29.0 \mathrm{mmol})$. A portion of the alkyne mixture $(3 \mathrm{~mL})$ was added to the zinc butanol slurry along with a few crystals of iodine and 1,2-dibromoethane $(2.50 \mathrm{~mL}, 29.0 \mathrm{mmol})$. The mixture was then heated cautiously (using a heat gun) with stirring until the reaction had clearly commenced. Slow addition of the remaining alkyne mixture followed, with external heating applied to maintain the reaction. The crude product was collected by distillation from the reaction mixture during the course of the reaction, and external heat applied to continue collection up to a temperature of 44 ${ }^{\circ} \mathrm{C}$. The crude distillate was redistilled to give 1,1-dimethylallene $(17.5 \mathrm{~g}, 257 \mathrm{mmol}, 66 \%)$ as a colourless liquid. All spectroscopic data matched literature values. ${ }^{1}$<smiles>C=C(C)C</smiles>

BP $40-42{ }^{\circ} \mathrm{C}$;

${ }^{1} \mathbf{H}$ NMR $\left(500 \mathrm{MHz}, \mathrm{CDCl}_{3}\right) \delta 4.52$ (hept, $\left.J=3.1 \mathrm{~Hz}, 2 \mathrm{H}\right), 1.69(\mathrm{t}, J=3.2 \mathrm{~Hz}, 6 \mathrm{H}) \mathrm{ppm}$;

${ }^{13} \mathbf{C}$ NMR $\left(151 \mathrm{MHz}, \mathrm{CDCl}_{3}\right) \delta 206.8,94.2,72.7,20.3 \mathrm{ppm}$.

Step (3) - A solution of $B$-Prenyl-9-BBN was prepared under anhydrous conditions, based on a procedure reported by Trauner and co-workers. ${ }^{66}$ A solution of $9-\mathrm{BBN}$ in THF $(360 \mathrm{~mL}$, $179 \mathrm{mmol}, 0.5 \mathrm{M}$ in THF) was cooled to $0{ }^{\circ} \mathrm{C}$. Once the solution was observed to go cloudy, 1,1dimethylallene $(20.5 \mathrm{~mL}, 208 \mathrm{mmol})$ was added dropwise over 30 minutes with stirring. The reaction was warmed to room temperature and stirred for $18 \mathrm{~h}$ before being used directly in the reverse prenylation procedure described on page 15 . 


\section{References:}

1. Chengebroyen, J., Linke, M., Robitzer, M., Sirlin, C. \& Pfeffer, M. Palladium-mediated intramolecular $\mathrm{C}-\mathrm{N}$ bond formation involving allyl substituted pyridines. Application to a novel strategy for the synthesis of the skeleton of berberinium derivatives. J. Organomet. Chem. 687, 313-321 (2003).

2. Kuttruff, C. A., Zipse, H. \& Trauner, D. Concise Total Syntheses of Variecolortides A and B through an Unusual Hetero-Diels-Alder Reaction. Angew. Chem. Int. Ed. 50, 1402-1405 (2011). 
3.12 Experimental Procedure for $t$ - $\mathrm{BuOCl}$

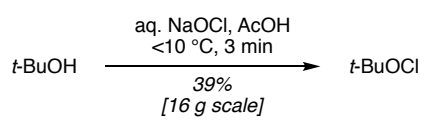

$t$ - $\mathrm{BuOCl}$ was prepared based on a procedure reported by Mintz and co-workers. ${ }^{1}$ For safety reasons exposure of the reaction and product to light was minimised.

An aqueous solution of bleach $(500 \mathrm{~mL}, 0.767 \mathrm{M}, 384 \mathrm{mmol})^{*}$ was cooled below $10{ }^{\circ} \mathrm{C}$ with stirring. A solution of $t$-BuOH $(43.3 \mathrm{~mL}, 383 \mathrm{mmol})$ and glacial acetic acid $(24.1 \mathrm{~mL}, 421 \mathrm{mmol})$ was added in a single portion to the rapidly stirred bleach and the stirring continued for $3 \mathrm{~min}$. The entire reaction mixture was transferred to a separating funnel, the aqueous layer discarded, the yellow organic layer washed with $10 \%$ aq. $\mathrm{Na}_{2} \mathrm{CO}_{3}(50 \mathrm{~mL})$ and water $(50 \mathrm{~mL})$. The product was dried $\left(\mathrm{CaCl}_{2}\right.$ approximately $\left.3 \mathrm{~g}\right)$ and decanted into its final container via pipette. The $t$-BuOCl (16.4 g, $151 \mathrm{mmol}, 39 \%$ ) was stored in a fridge over $\mathrm{CaCl}_{2}$ in an amber glass bottle as a yellow liquid.

*Bleach solution was titrated to a concentration of $0.767 \mathrm{M}$ by iodometric titration using sodium thiosulfate prior to use.

${ }^{1} \mathbf{H}$ NMR $\left(500 \mathrm{MHz}, \mathrm{CDCl}_{3}\right) \delta 1.33(\mathrm{~s}, 9 \mathrm{H}) \mathrm{ppm}$;

${ }^{13} \mathbf{C ~ N M R}\left(126 \mathrm{MHz}, \mathrm{CDCl}_{3}\right) \delta 84.1,27.0 \mathrm{ppm}$.

\section{References:}

1. Mintz, M. J. \& Walling, C. $t$-BUTYL HYPOCHLORITE. Org. Synth. 49, 9 (1969). 
4.1 ${ }^{1} \mathrm{H}$ NMR Spectrum of Compound $14\left(500 \mathrm{MHz}, \mathrm{CDCl}_{3}\right)$

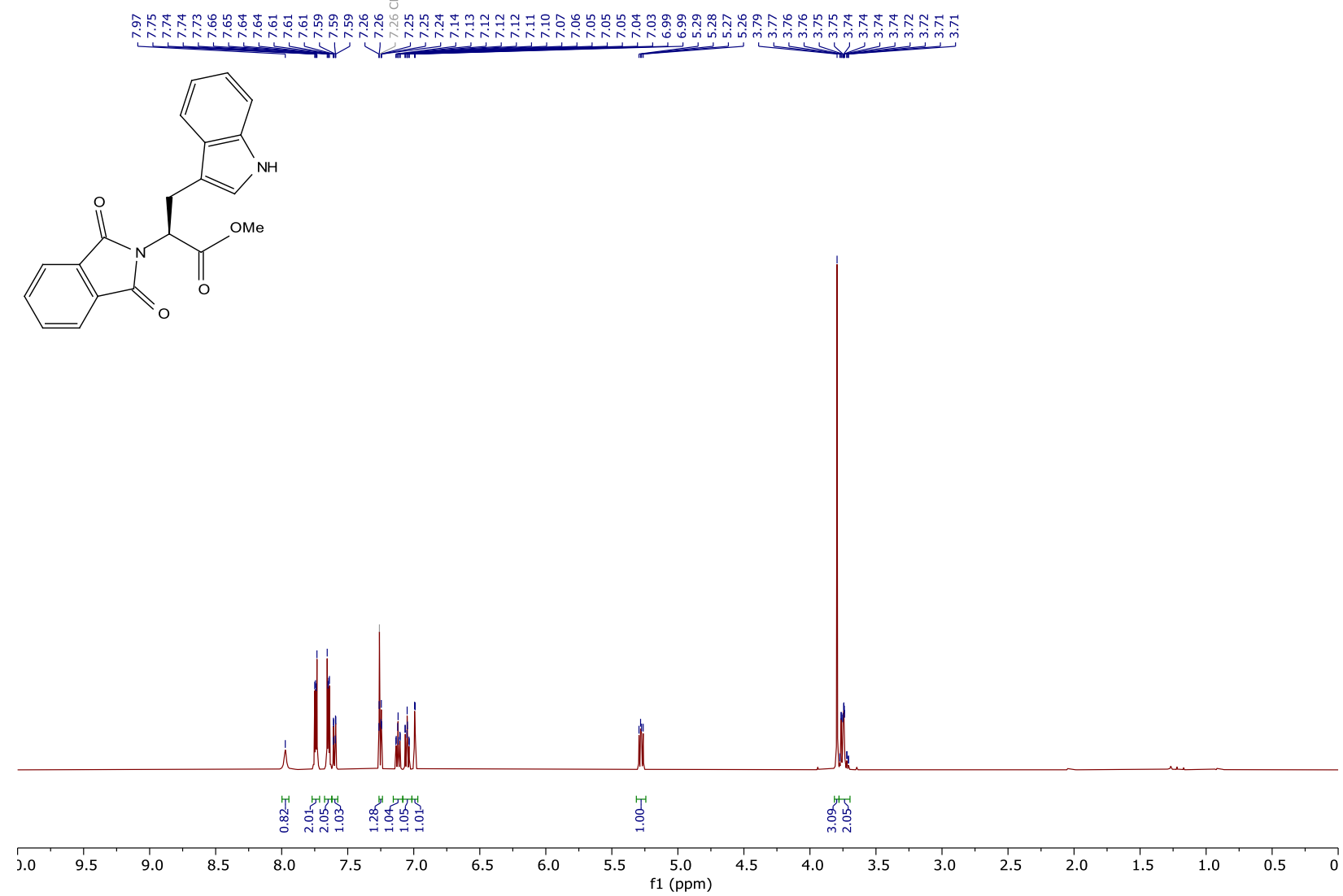

4.2 ${ }^{13} \mathrm{C}$ NMR Spectrum of Compound $14\left(126 \mathrm{MHz}, \mathrm{CDCl}_{3}\right)$
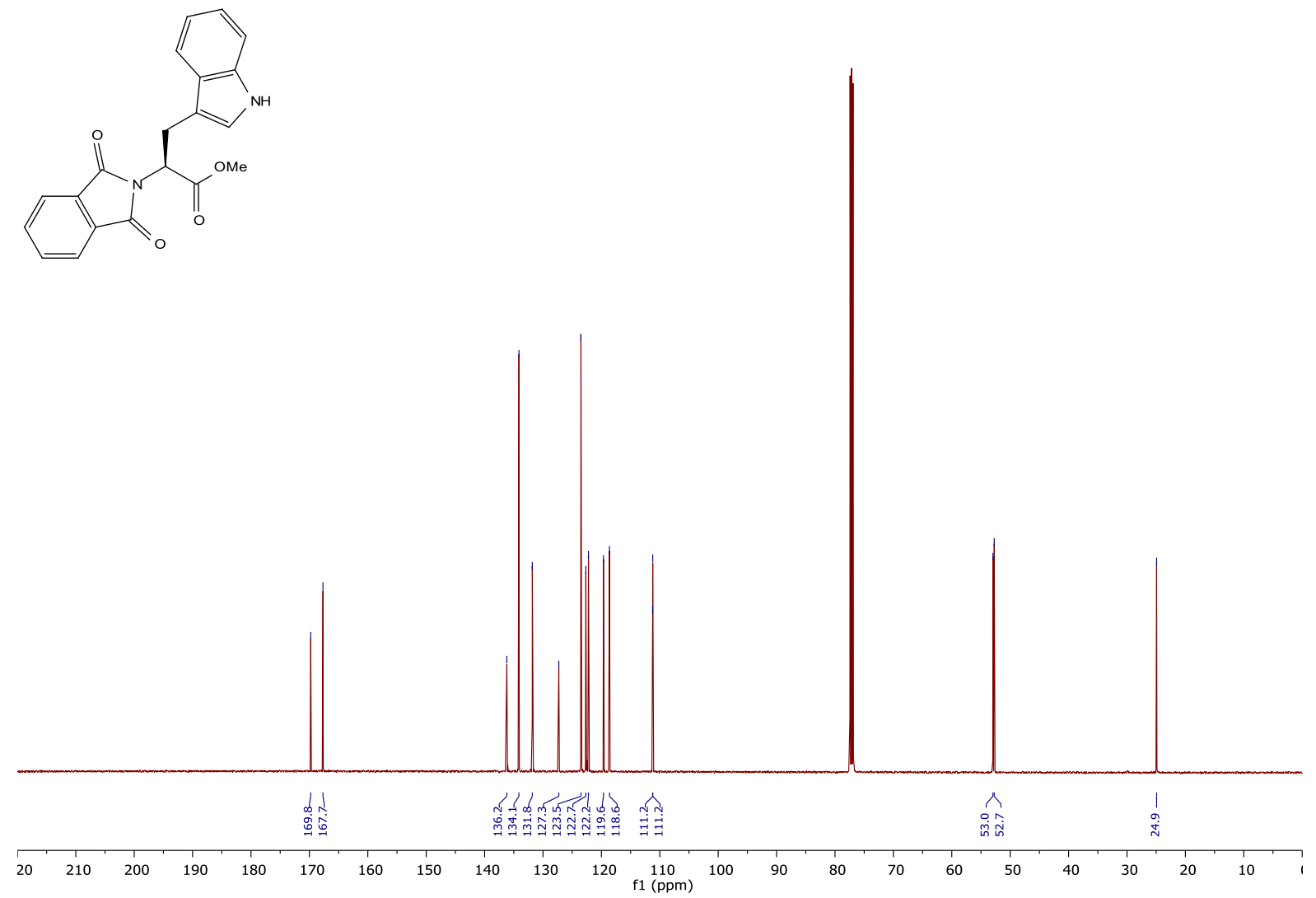
4.3 ${ }^{1} \mathrm{H}$ NMR Spectrum of Compound $15\left(600 \mathrm{MHz}, \mathrm{CDCl}_{3}\right)$

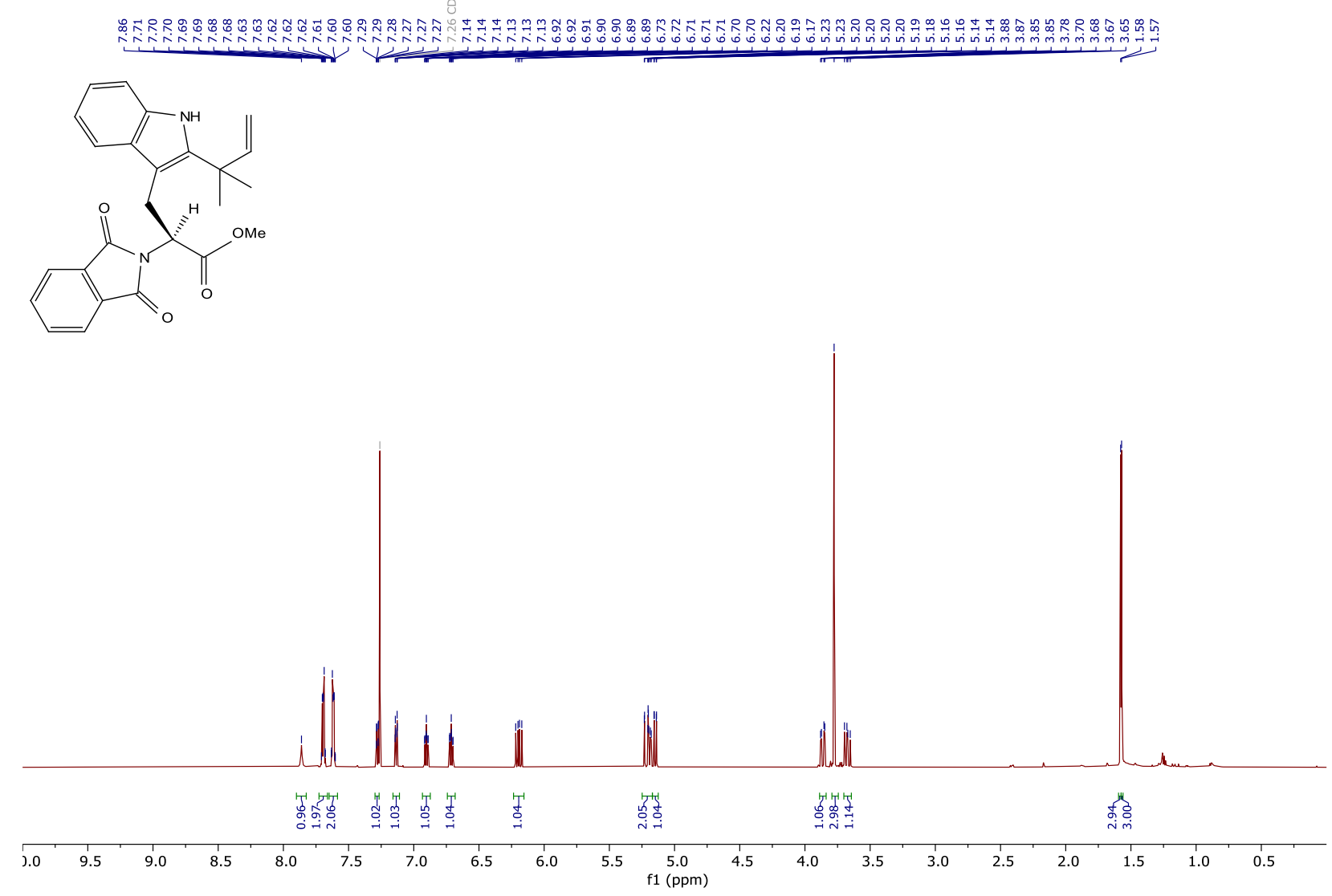

4.4 ${ }^{13} \mathrm{C}$ NMR Spectrum of Compound $15\left(151 \mathrm{MHz}, \mathrm{CDCl}_{3}\right)$
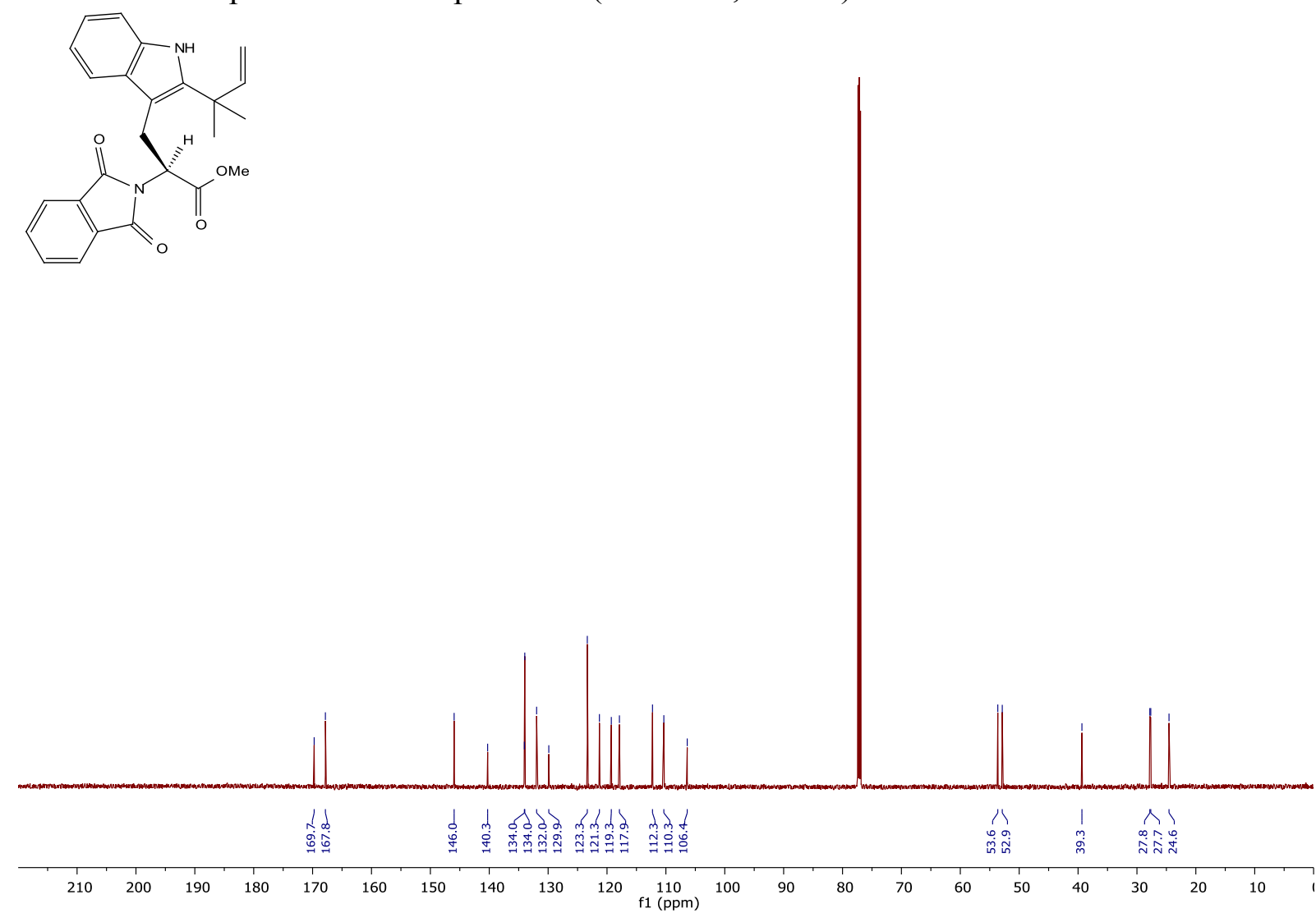

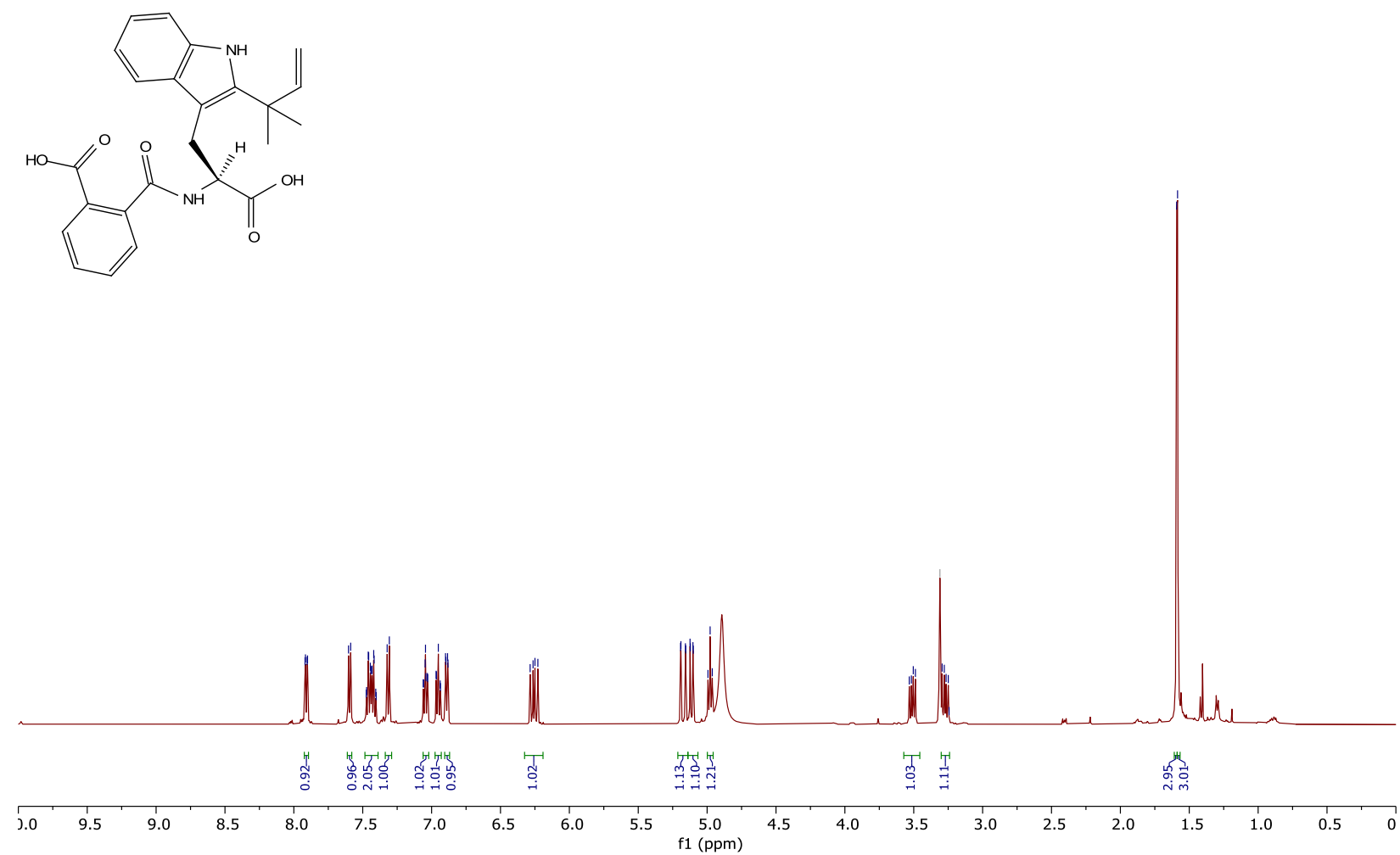

4.6 ${ }^{13} \mathrm{C}$ NMR Spectrum of Compound $16\left(126 \mathrm{MHz}, \mathrm{CD}_{3} \mathrm{OD}\right)$
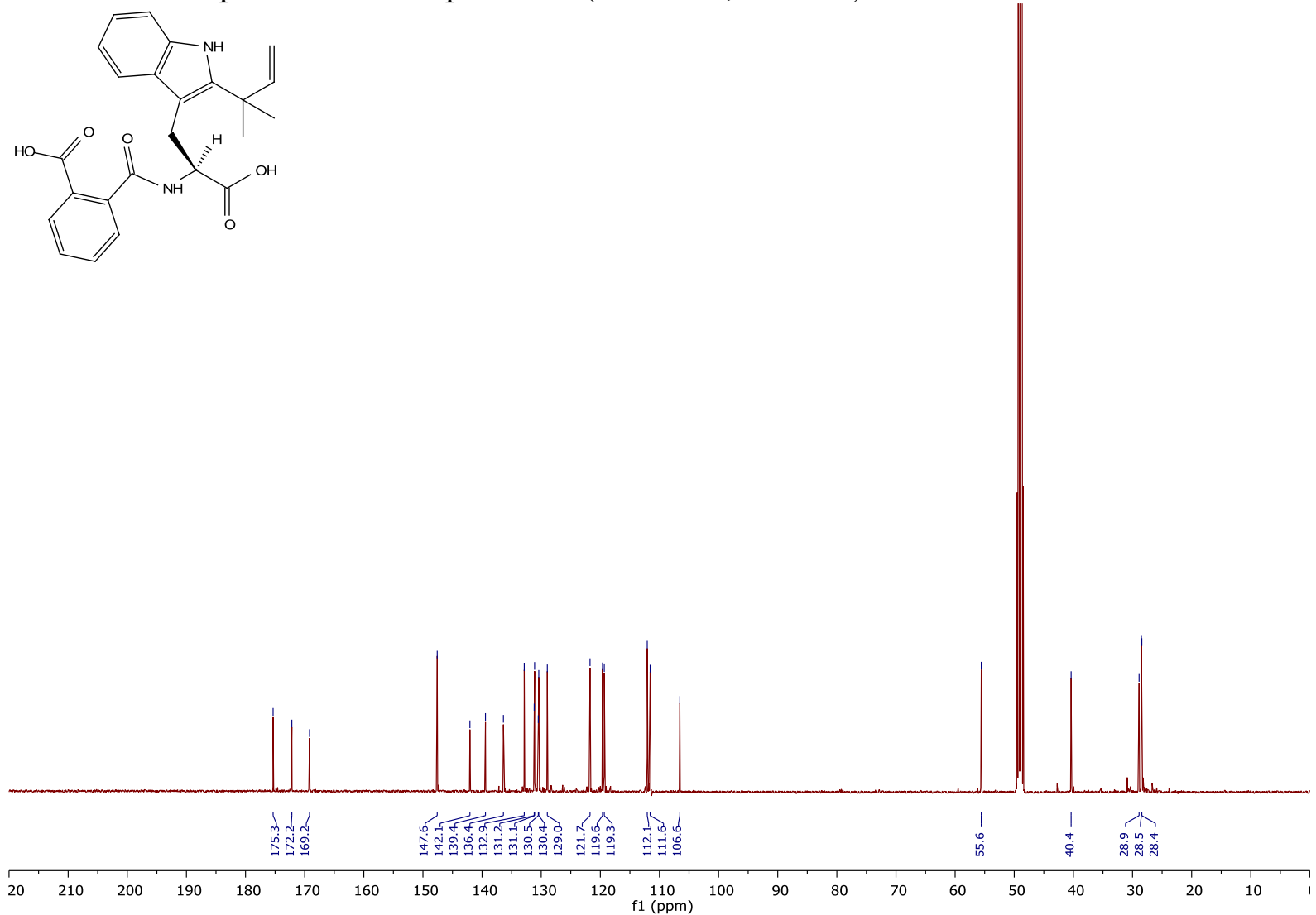
4.7 ${ }^{1} \mathrm{H}$ NMR Spectrum of Compound $17\left(500 \mathrm{MHz}, \mathrm{CD}_{3} \mathrm{OD}\right)$

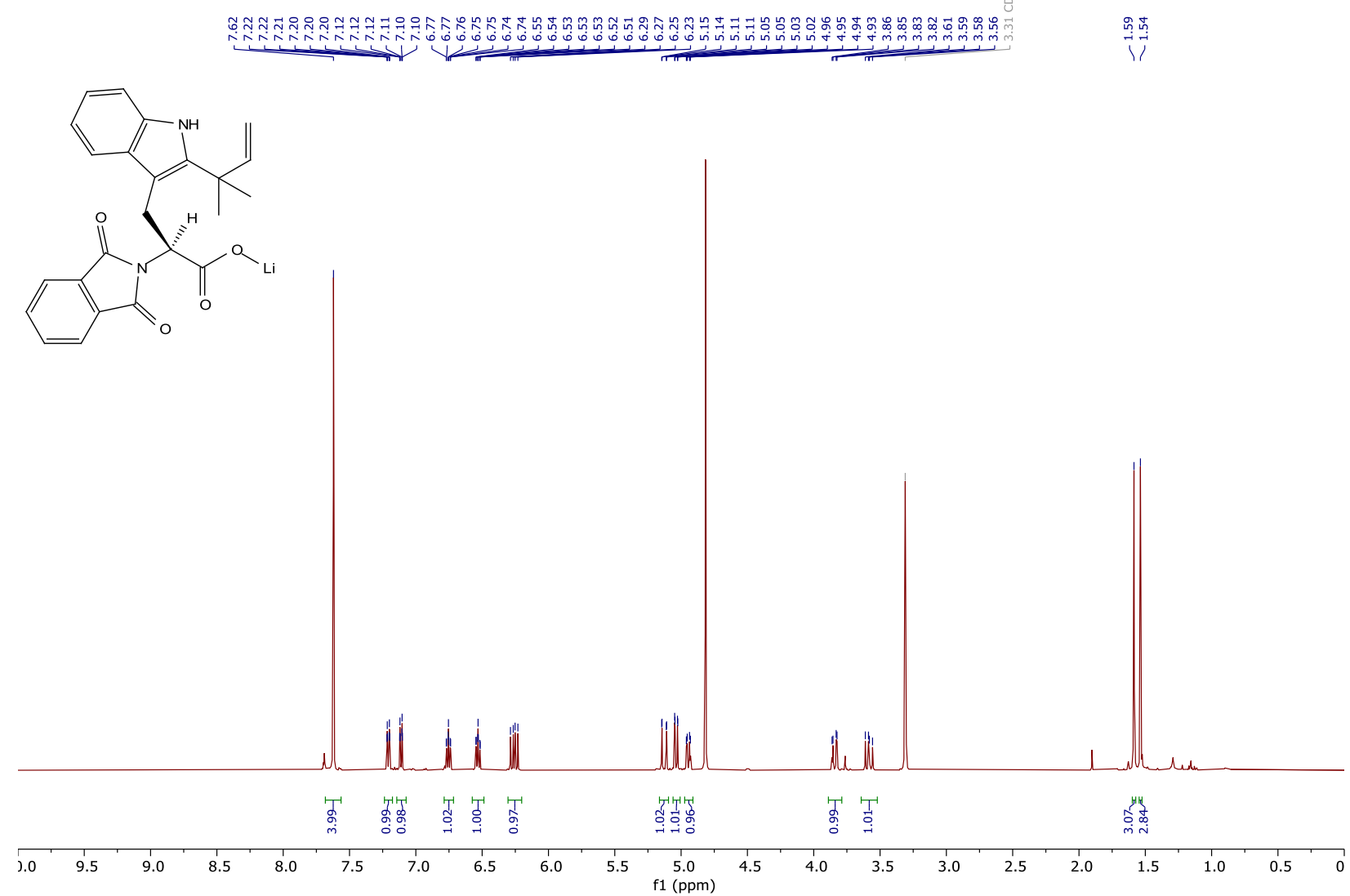

4.8 ${ }^{13} \mathrm{C}$ NMR Spectrum of Compound $17\left(126 \mathrm{MHz}, \mathrm{CD}_{3} \mathrm{OD}\right)$
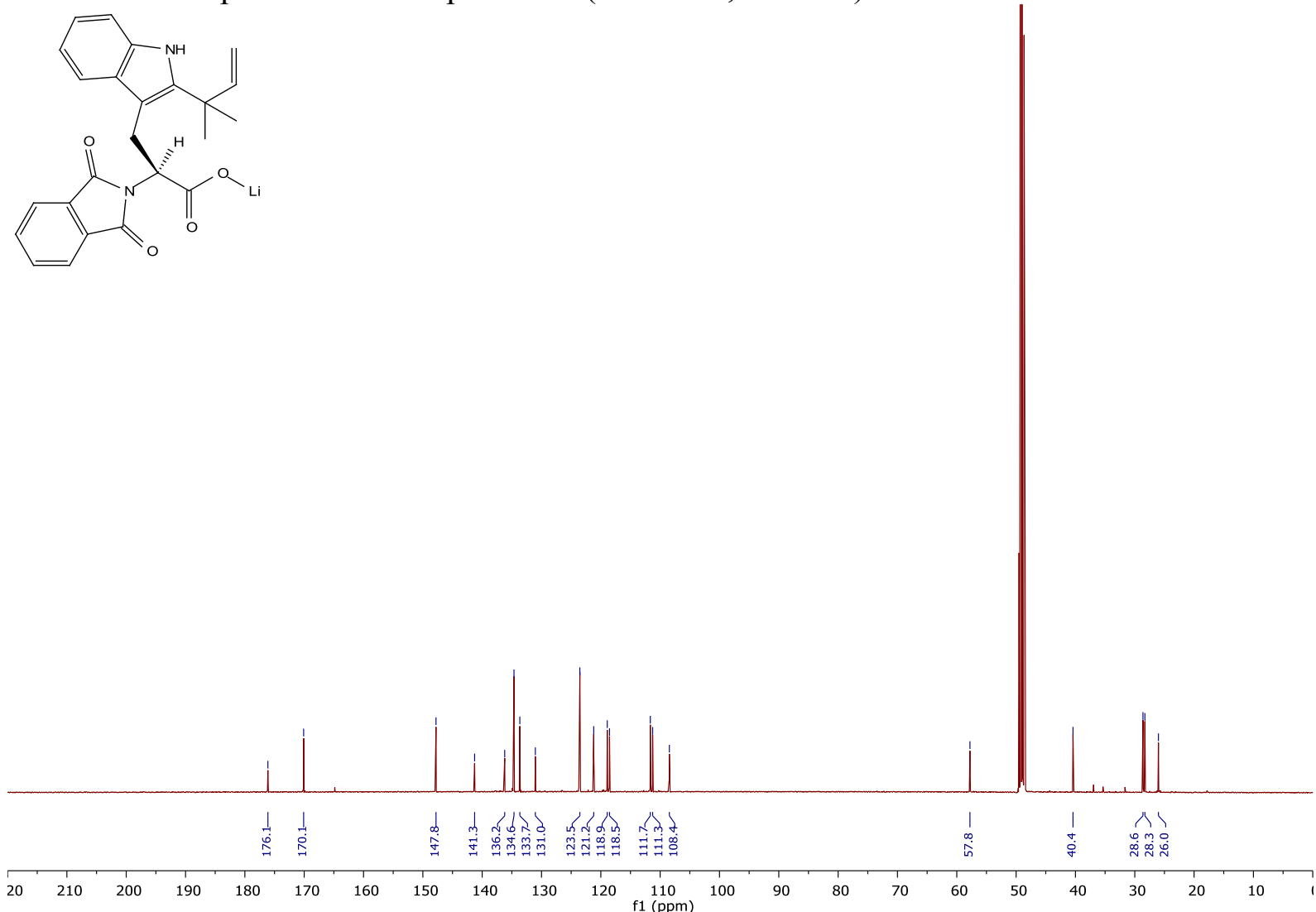
4.9 ${ }^{1} \mathrm{H}$ NMR Spectrum of Compound $19\left(500 \mathrm{MHz}, \mathrm{CD}_{2} \mathrm{Cl}_{2}\right)$

年
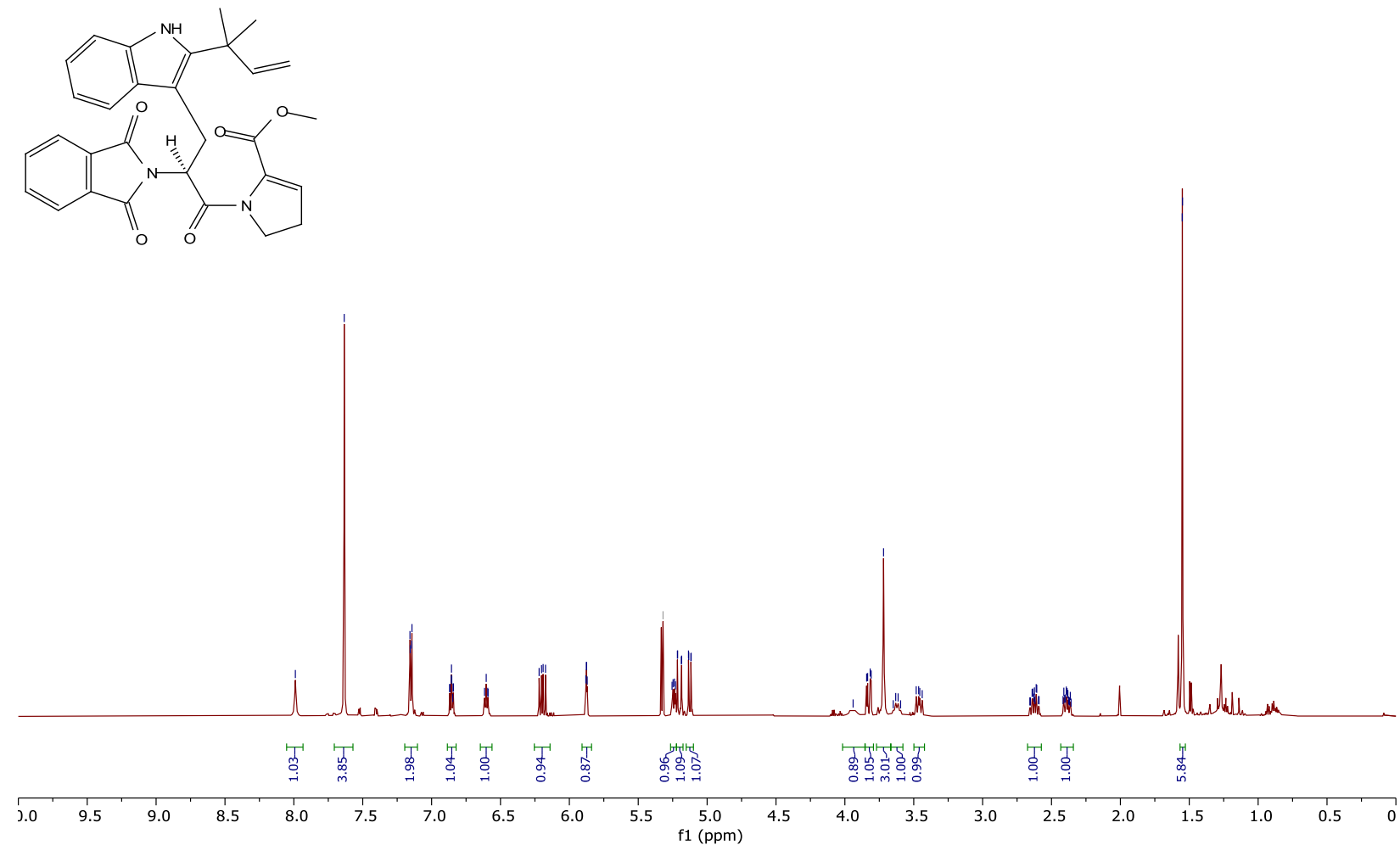

4.10 ${ }^{13} \mathrm{C}$ NMR Spectrum of Compound $19\left(151 \mathrm{MHz}, \mathrm{CD}_{2} \mathrm{Cl}_{2}\right)$
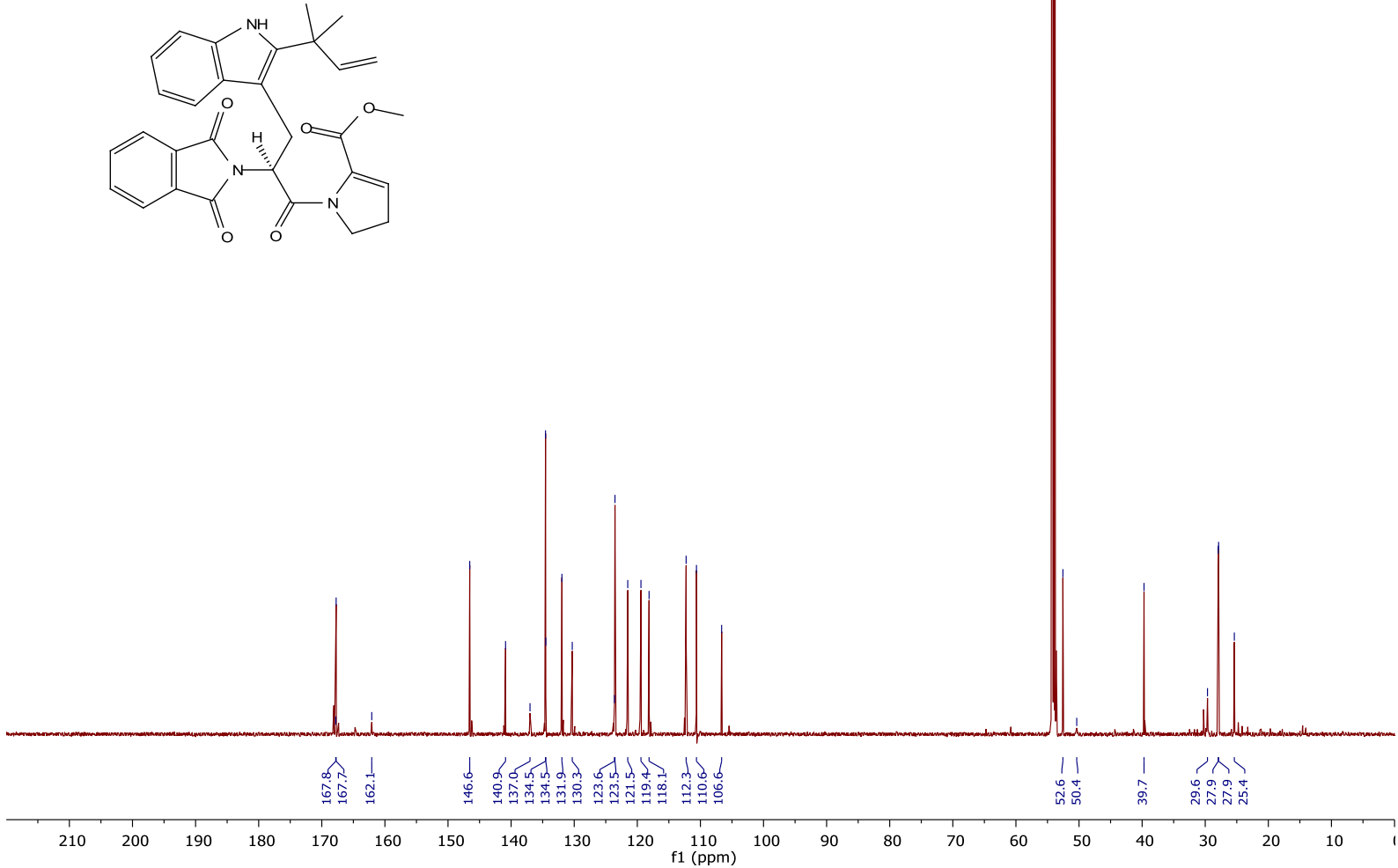
4.11 ${ }^{1} \mathrm{H}$ NMR Spectrum of Compound $9\left(500 \mathrm{MHz}, \mathrm{CDCl}_{3}\right)$

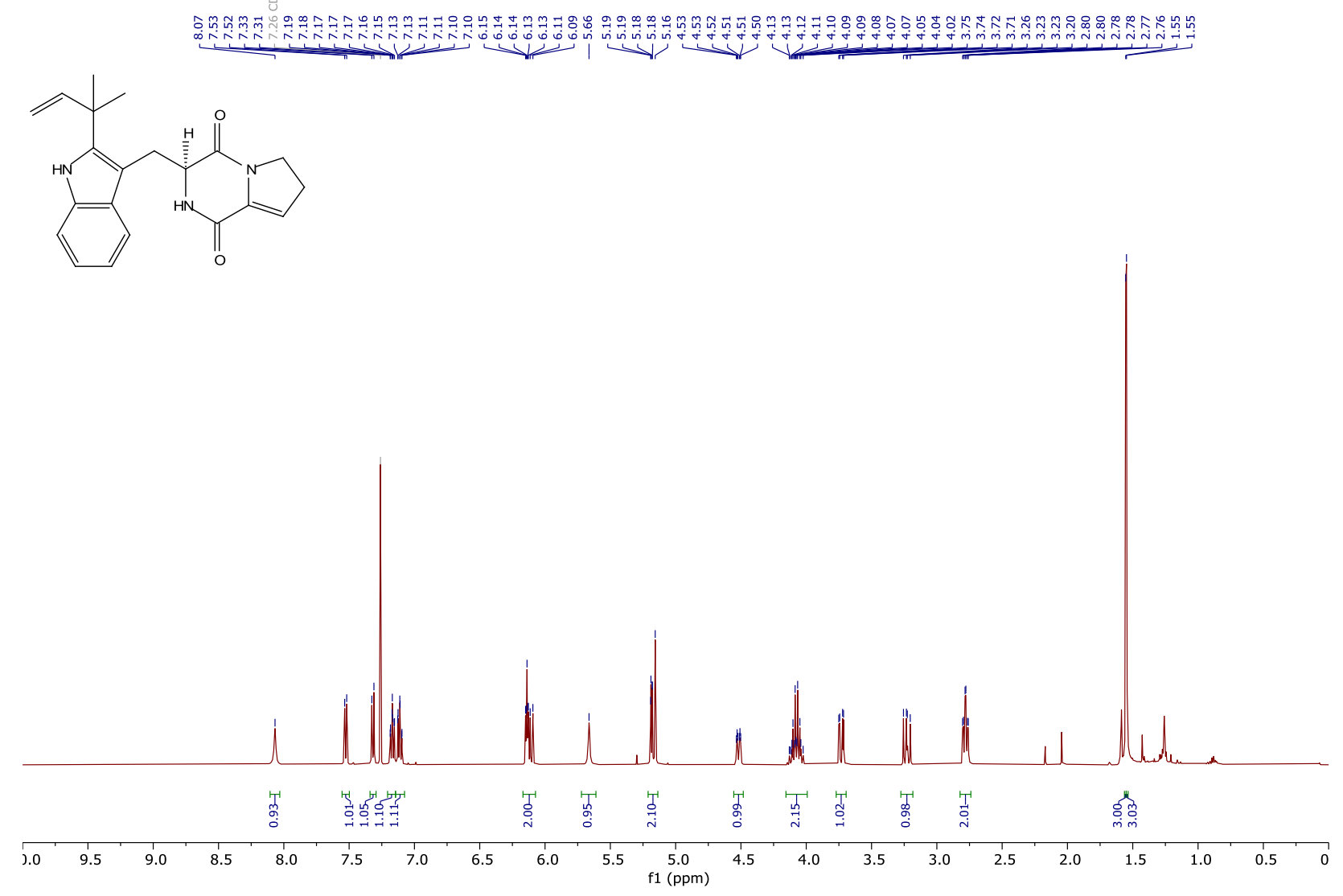

4.12 ${ }^{13} \mathrm{C}$ NMR Spectrum of Compound $9\left(126 \mathrm{MHz}, \mathrm{CDCl}_{3}\right)$<smiles>C=CC(C)(C)c1[nH]c2ccccc2c1C[C@H]1NC(=O)C2=CCCN2C1=O</smiles>

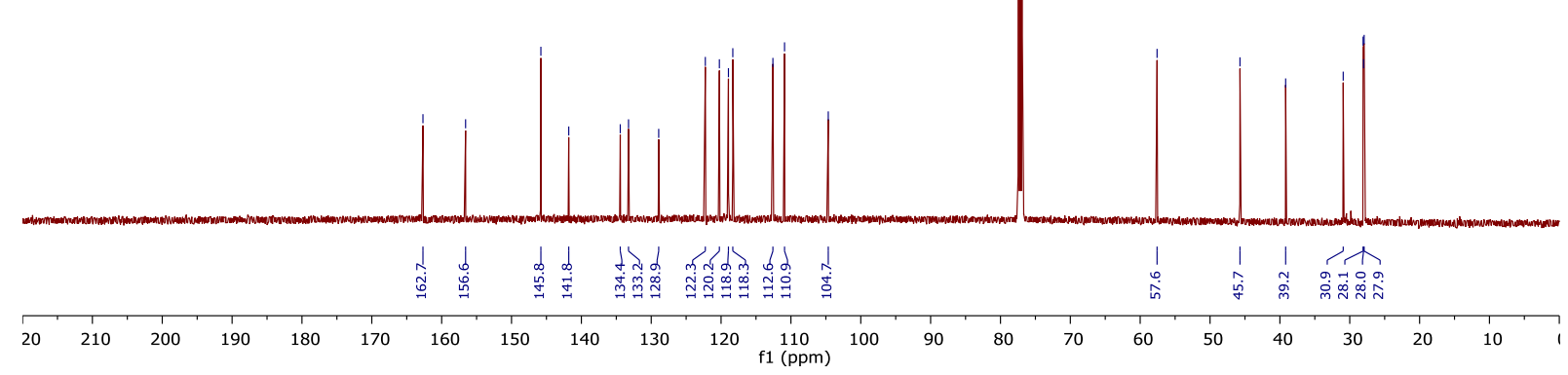


4.13 ${ }^{1} \mathrm{H}$ NMR Spectrum of Compound $12\left(500 \mathrm{MHz}, \mathrm{CDCl}_{3}\right)$

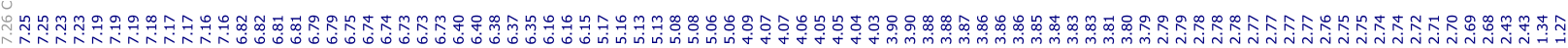<smiles>C=CC(C)(C)[C@]12Nc3ccccc3[C@@]1(O)C[C@H](O)C(=O)N1CCC=C1C2=O</smiles>

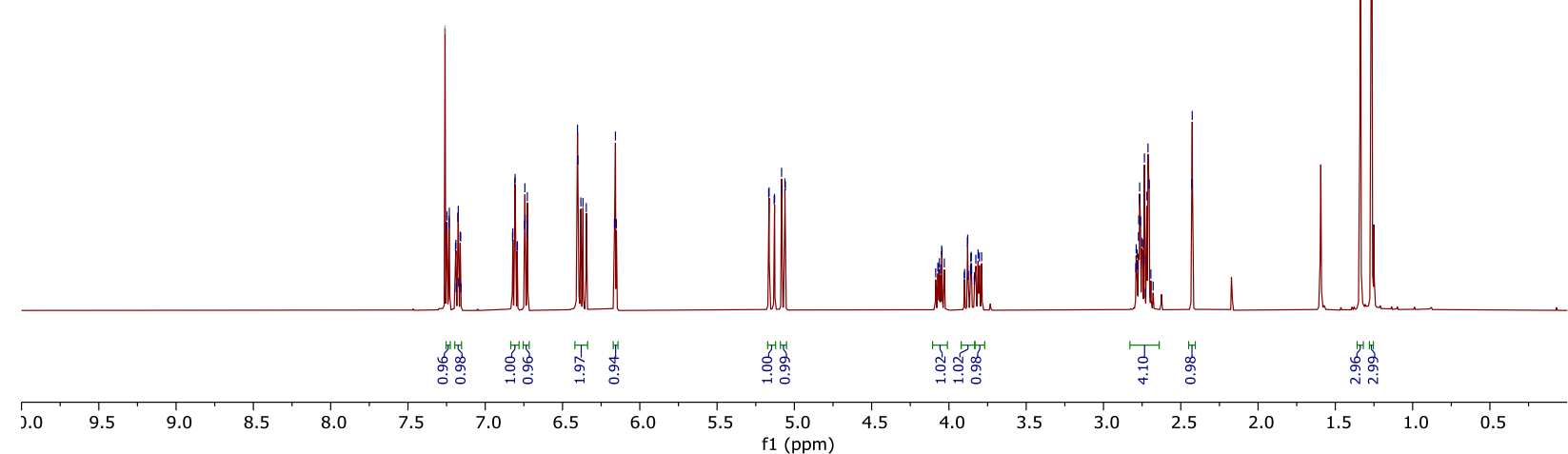

4.14 ${ }^{13} \mathrm{C}$ NMR Spectrum of Compound $12\left(126 \mathrm{MHz}, \mathrm{CDCl}_{3}\right)$<smiles>C=CC(C)(C)[C@]12Nc3ccccc3[C@]1(C)C[C@]1(C)C(=O)N3CCC=C3C(=O)N12</smiles>

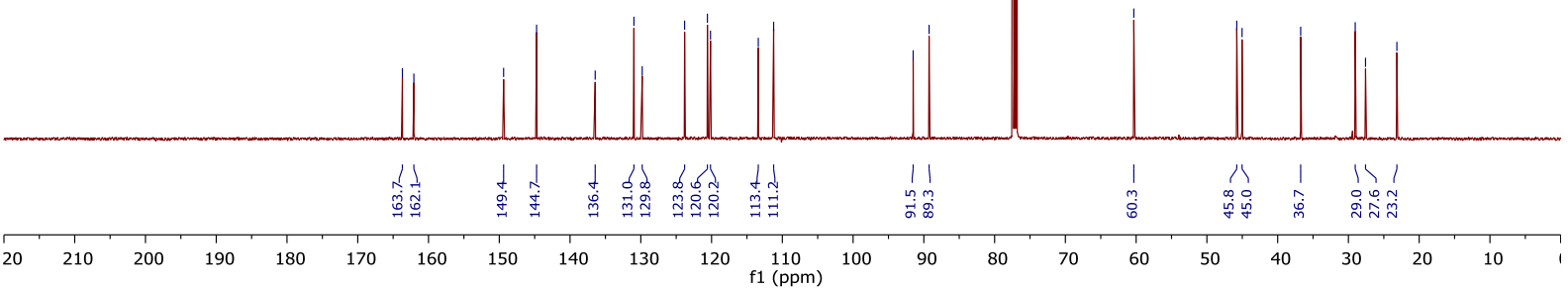


4.15 ${ }^{1} \mathrm{H}$ NMR Spectrum of Compound $20\left(500 \mathrm{MHz}, \mathrm{CDCl}_{3}\right)$

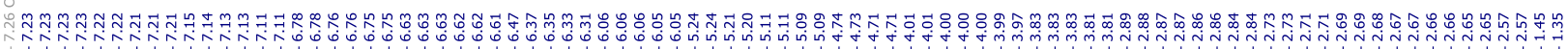

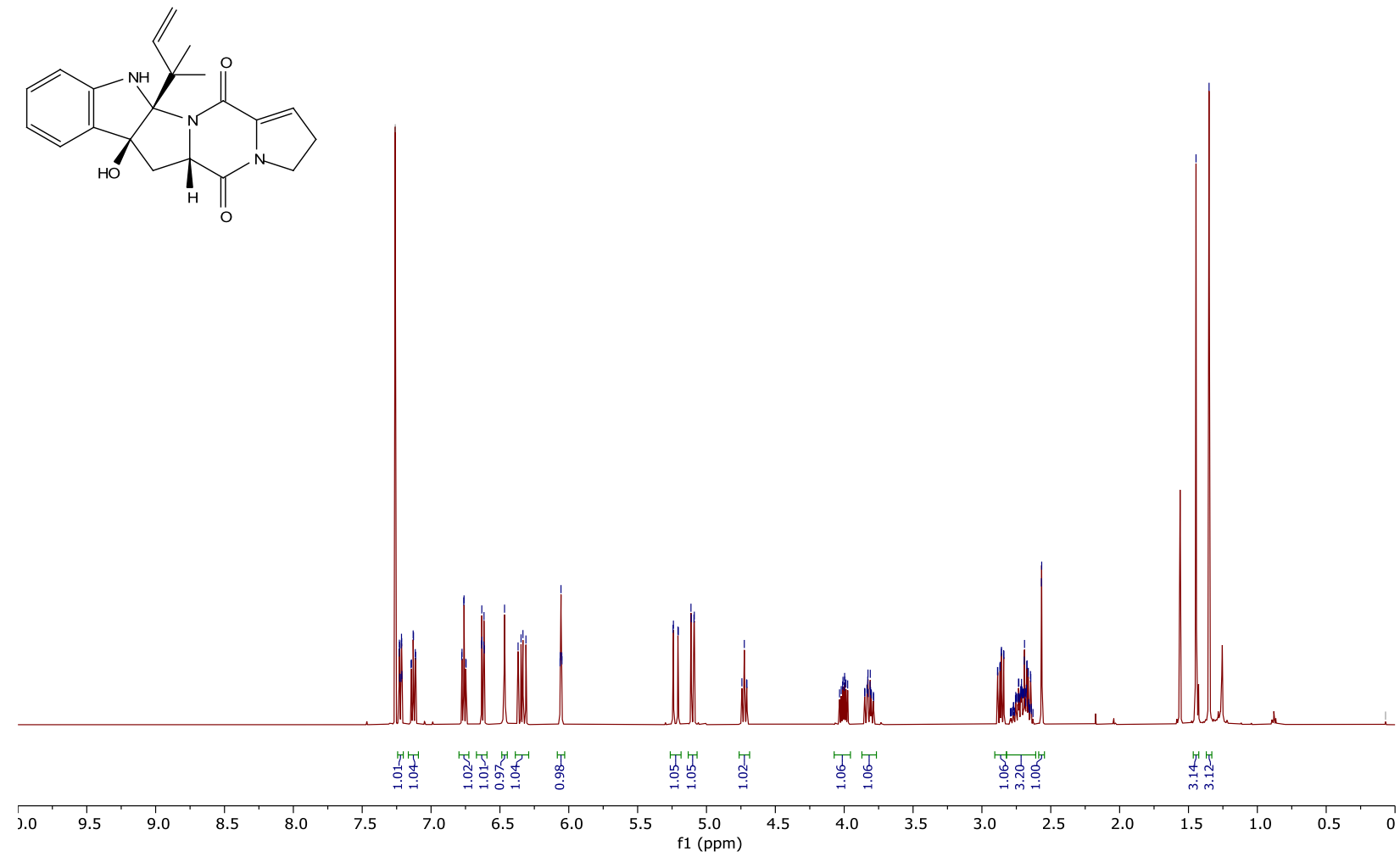

4.16 ${ }^{13} \mathrm{C}$ NMR Spectrum of Compound $20\left(126 \mathrm{MHz}, \mathrm{CDCl}_{3}\right)$

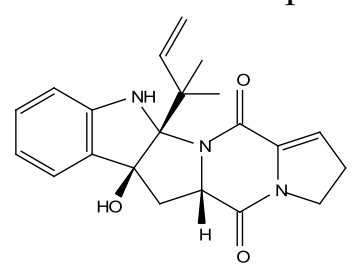

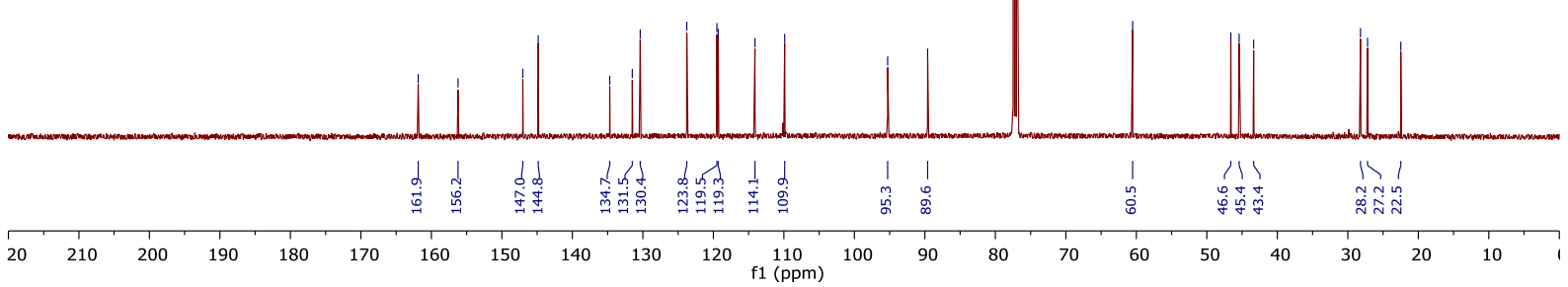


4.17 ${ }^{1} \mathrm{H}$ NMR Spectrum of Brevianamide A (1) $\left(500 \mathrm{MHz}, \mathrm{CDCl}_{3}, 3.0 \mathrm{mg} / \mathrm{mL}\right.$ )

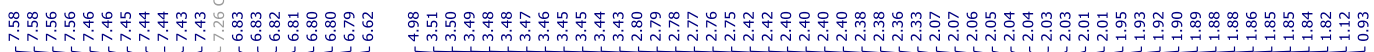
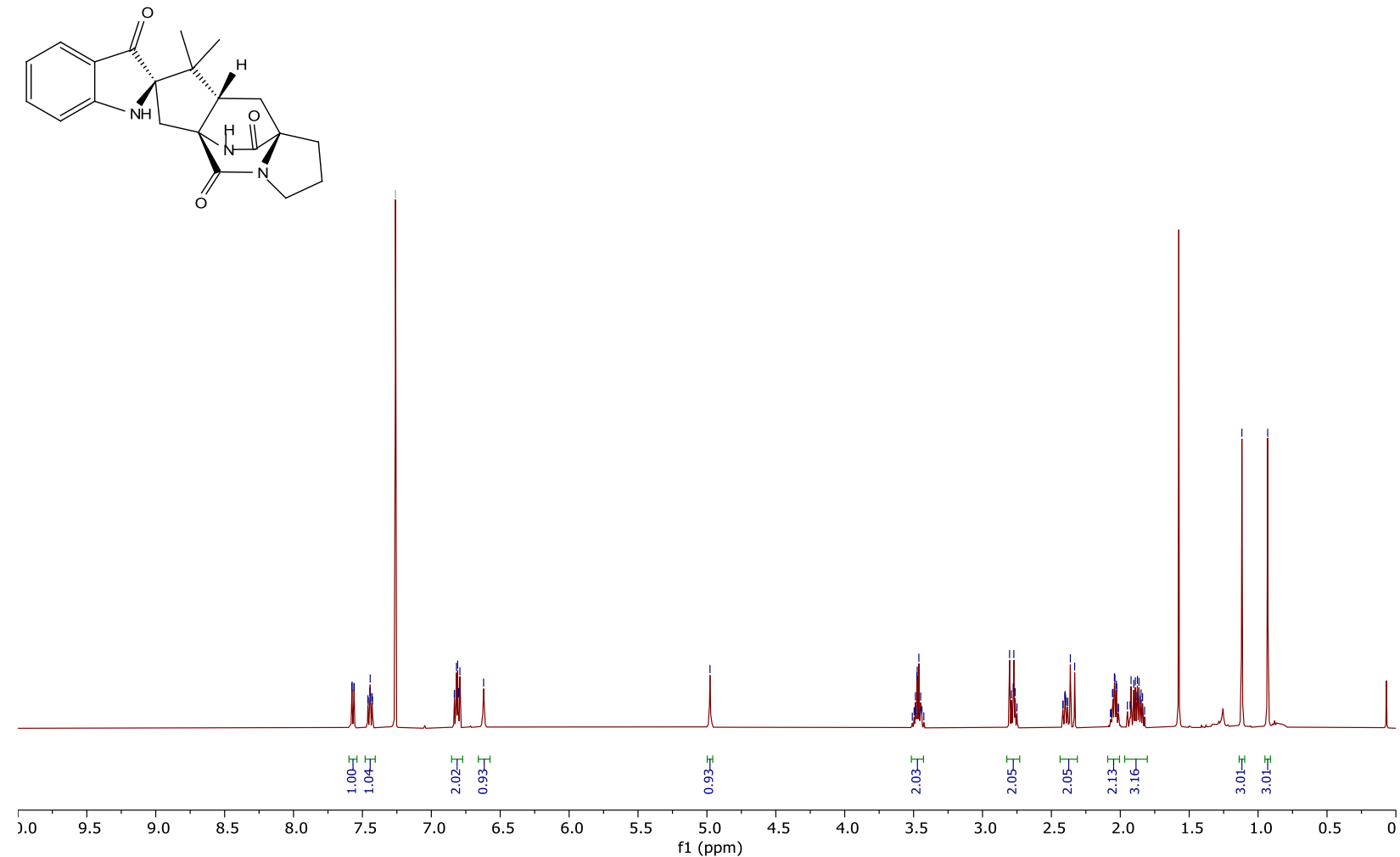

4.18 ${ }^{13} \mathrm{C}$ NMR Spectrum of Brevianamide A (1) $\left(126 \mathrm{MHz}, \mathrm{CDCl}_{3}, \sim 3.0 \mathrm{mg} / \mathrm{mL}\right)$
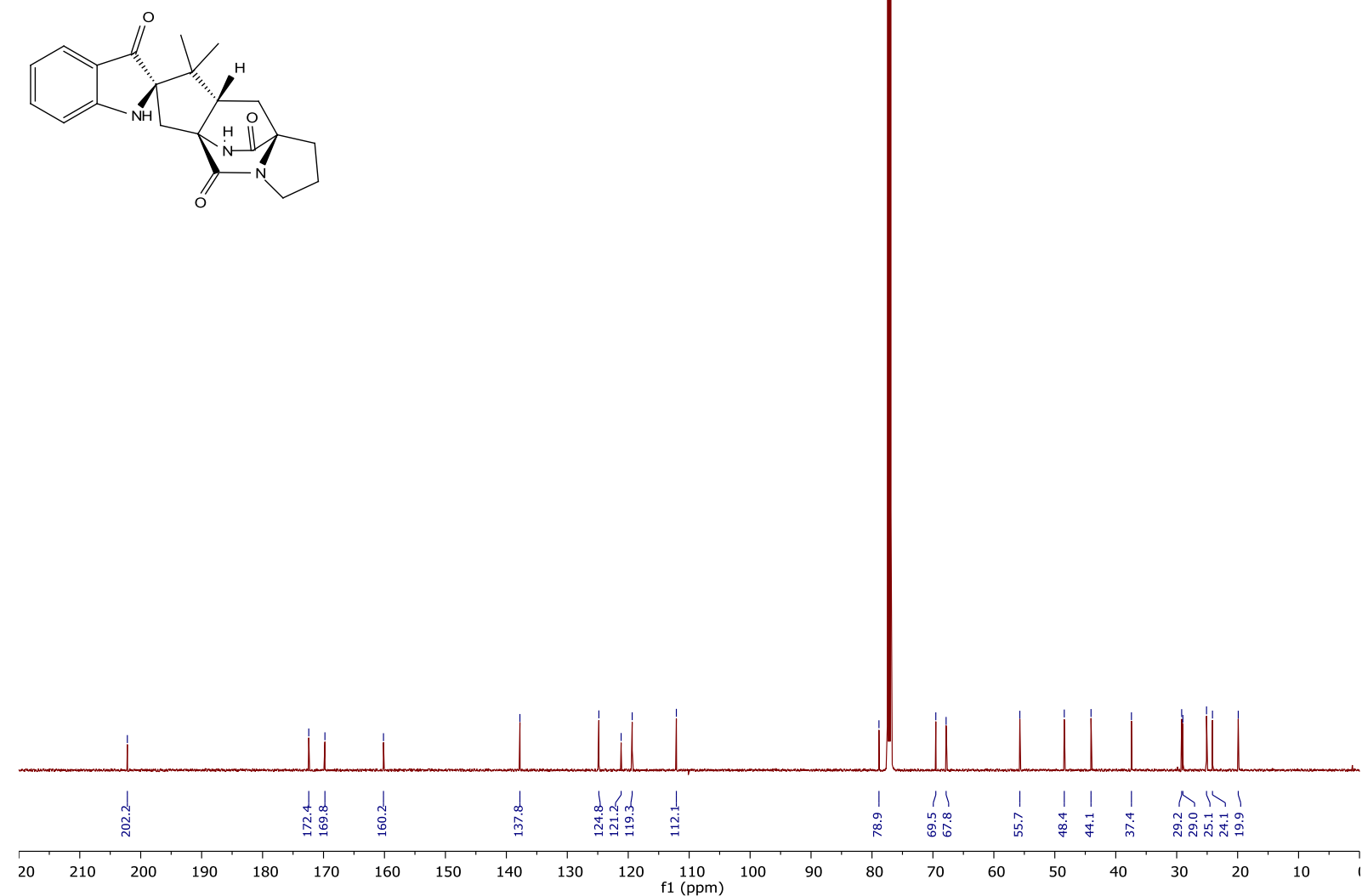
4.19 ${ }^{1} \mathrm{H}-{ }^{1} \mathrm{H}$ COSY Spectrum of Brevianamide A (1) $\left(\mathrm{CDCl}_{3}\right)$

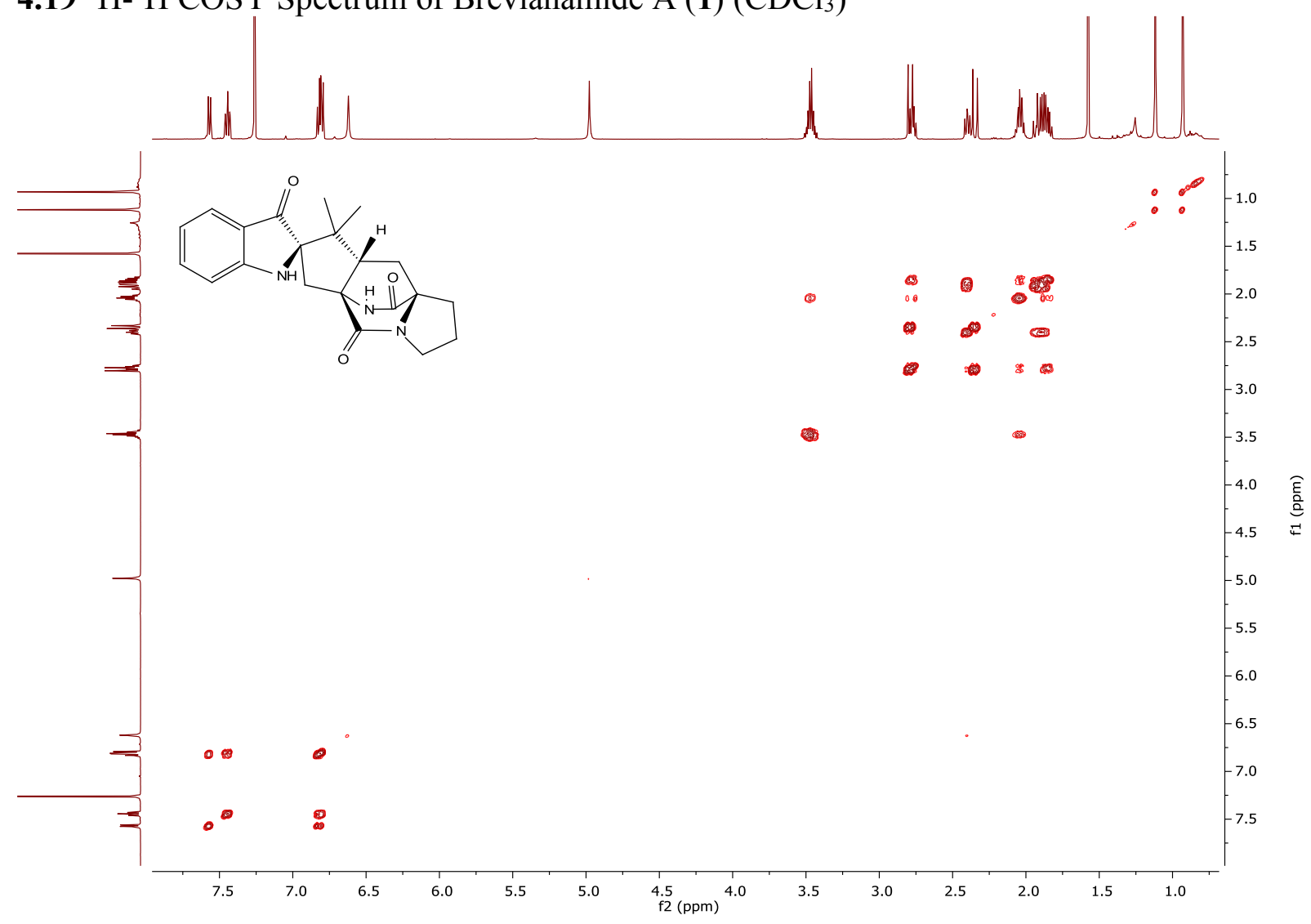

4.20 ${ }^{1} \mathrm{H}_{-}{ }^{13} \mathrm{C}$ HSQC Spectrum of Brevianamide A (1) $\left(\mathrm{CDCl}_{3}\right)$

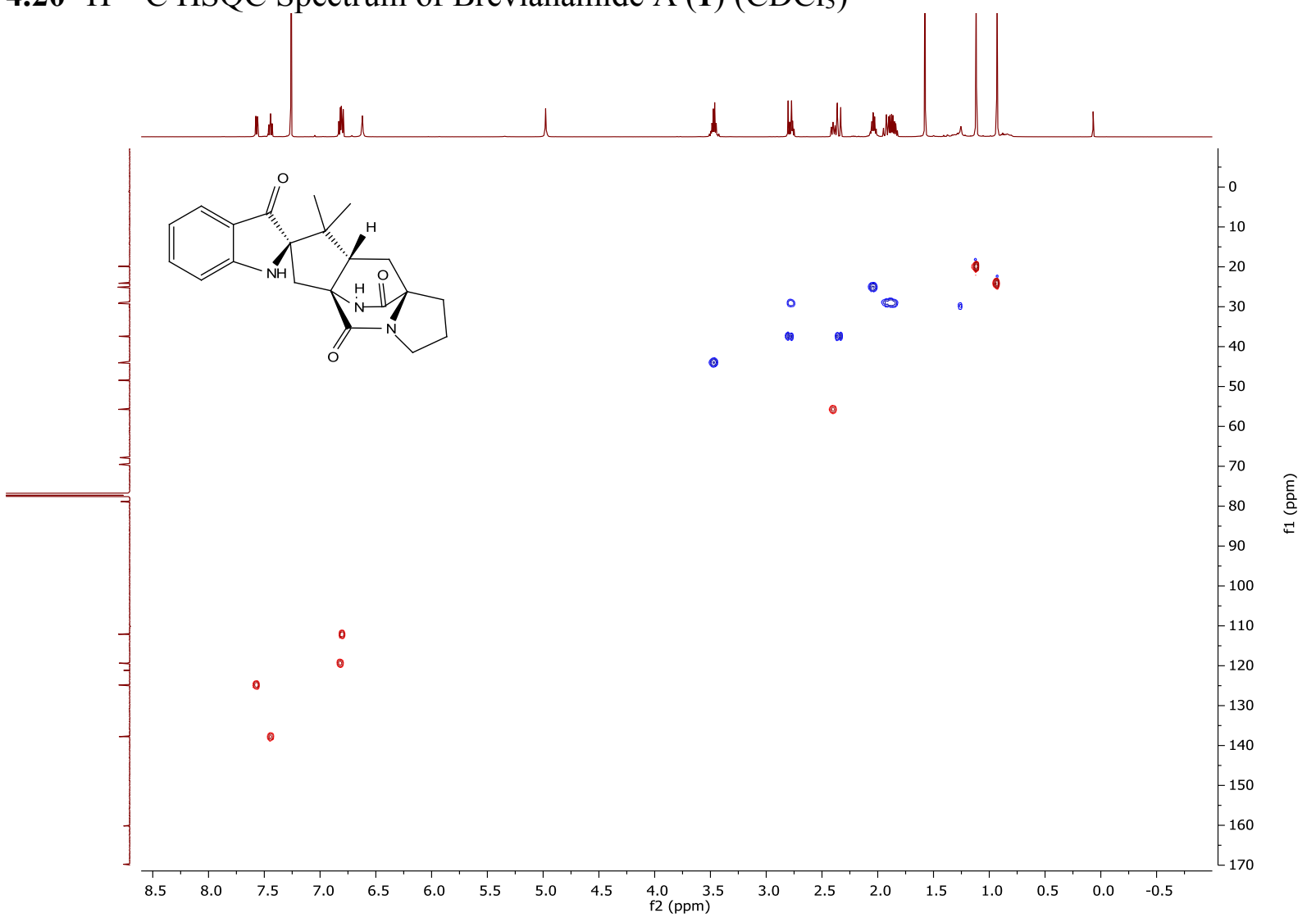


4.21 ${ }^{1} \mathrm{H}-{ }^{13} \mathrm{C}$ HMBC Spectrum of Brevianamide A (1) $\left(\mathrm{CDCl}_{3}\right)$

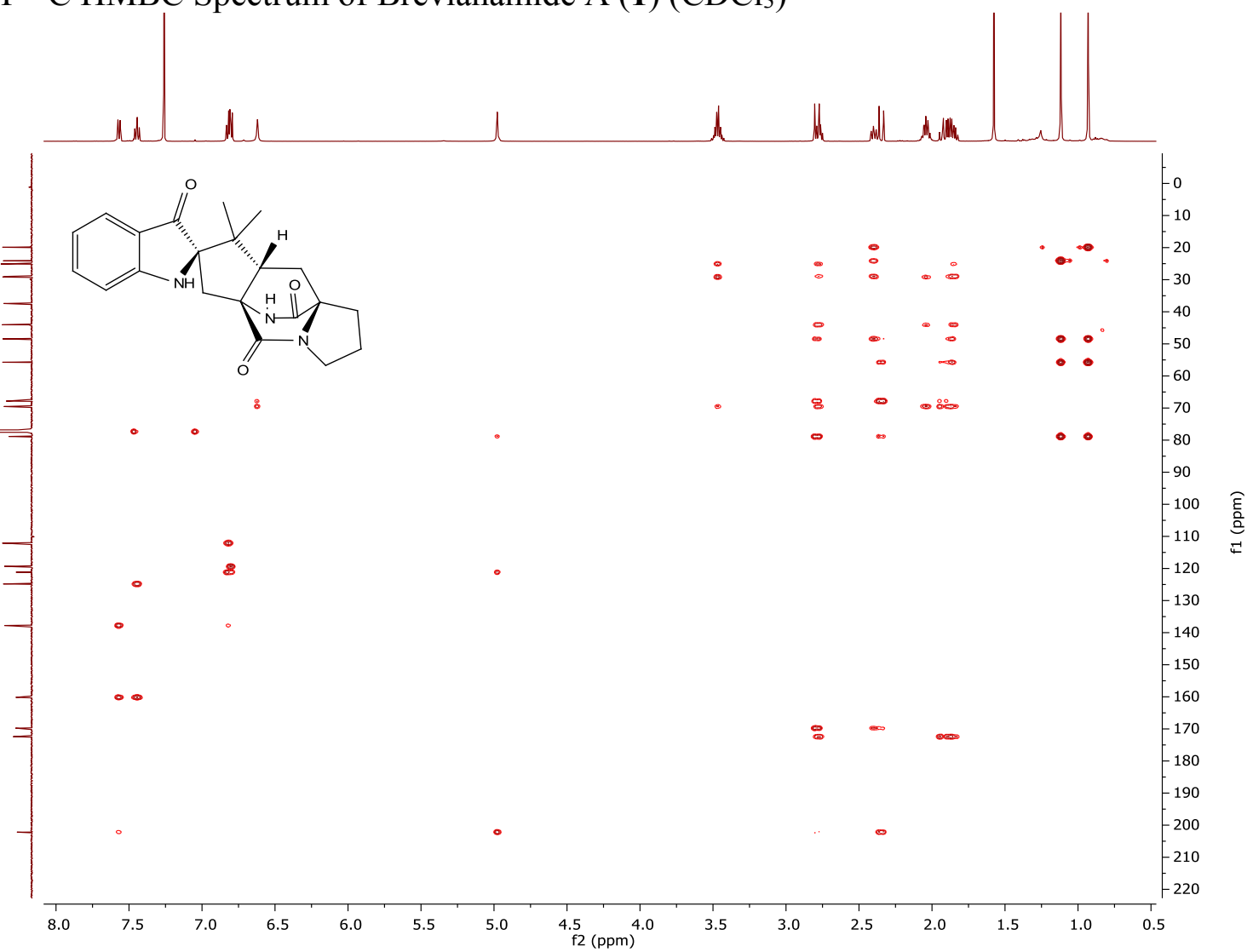

4.22 ${ }^{1} \mathrm{H}-{ }^{1} \mathrm{H}$ NOESY Spectrum of Brevianamide A (1) $\left(\mathrm{CDCl}_{3}\right)$

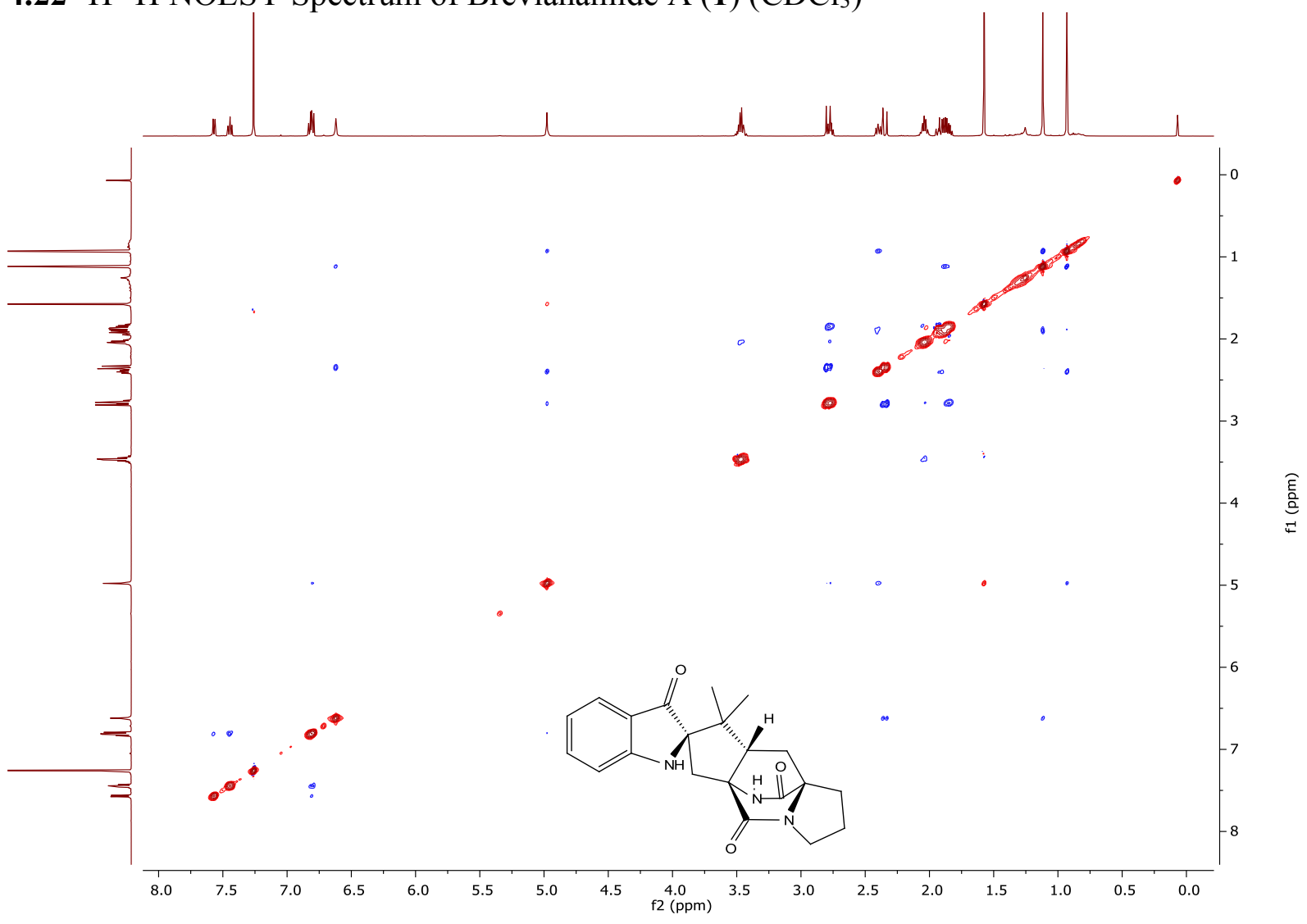

41 
4.23 ${ }^{1} \mathrm{H}$ NMR Spectra of Brevianamide A (1) (500 MHz, $\mathrm{CDCl}_{3}$ - conc. dependence)
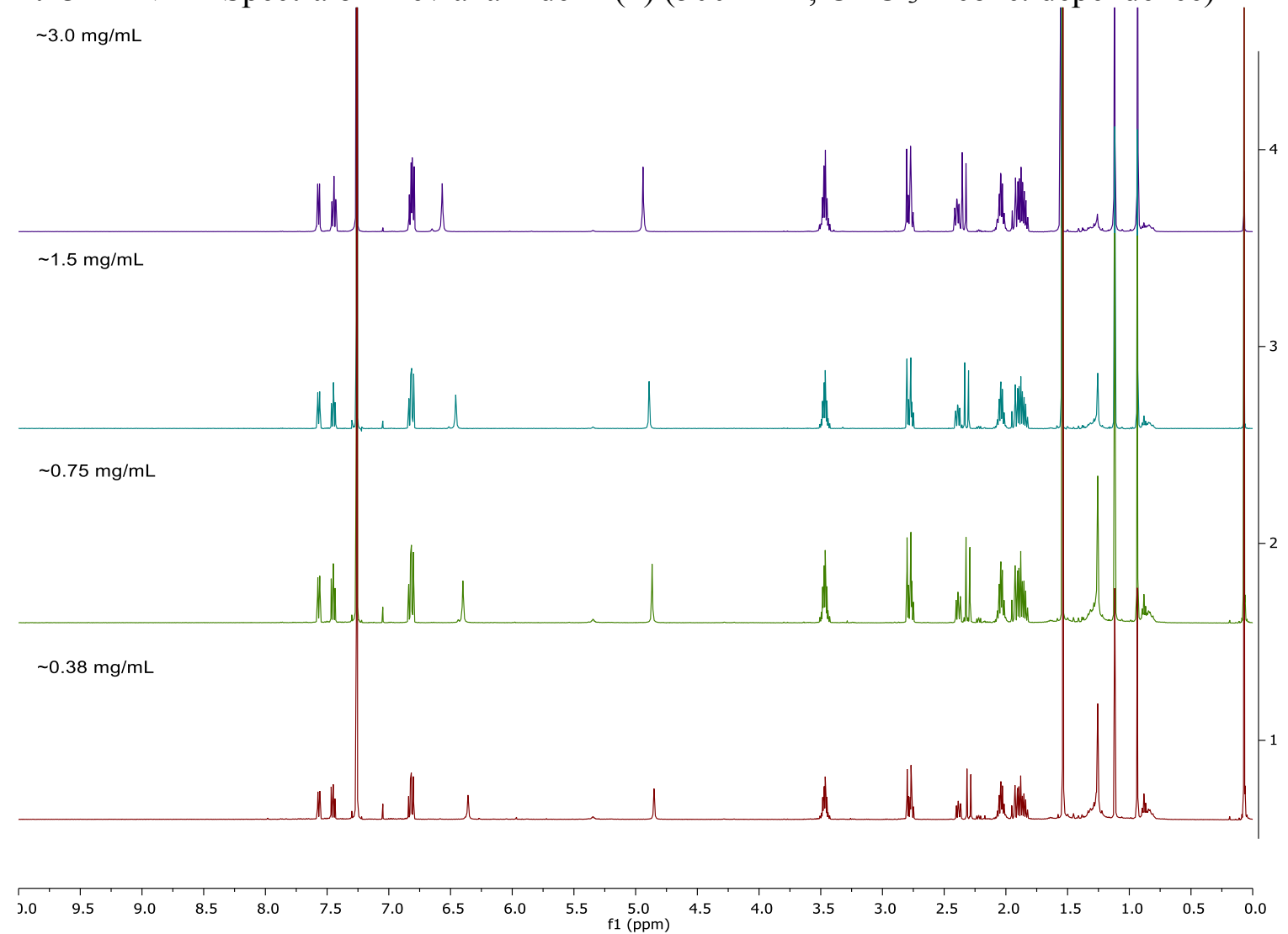

$\sim 3.0 \mathrm{mg} / \mathrm{mL}$
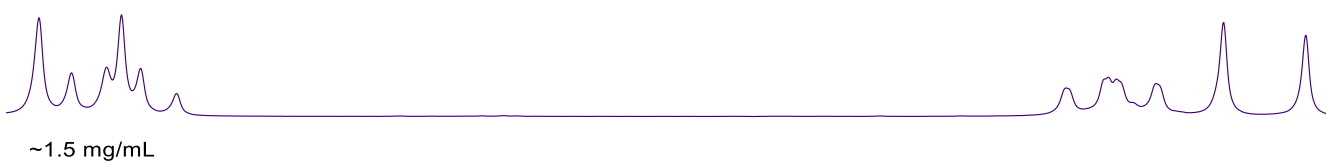

$$
\sim 1.5 \mathrm{mg} / \mathrm{mL}
$$

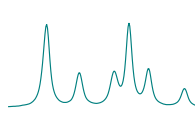

$\sim 0.75 \mathrm{mg} / \mathrm{mL}$

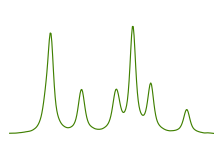

$\sim 0.38 \mathrm{mg} / \mathrm{mL}$

holh

$\begin{array}{llllllllllllllllllllllllllllllllllll}2.80 & 2.78 & 2.76 & 2.74 & 2.72 & 2.70 & 2.68 & 2.66 & 2.64 & 2.62 & 2.60 & 2.58 & 2.56 & 2.54 & 2.52 & 2.50 & 2.48 & 2.46 & 2.44 & 2.42 & 2.40 & 2.38 & 2.36 & 2.34 & 2.32 & 2.30 & 2.28 & 2.26\end{array}$ 
4.24 ${ }^{1} \mathrm{H}$ NMR Spectrum of Brevianamide A (1) (500 MHz, $\left.\left(\mathrm{CD}_{3}\right)_{2} \mathrm{SO}\right)$

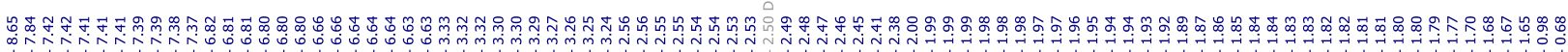
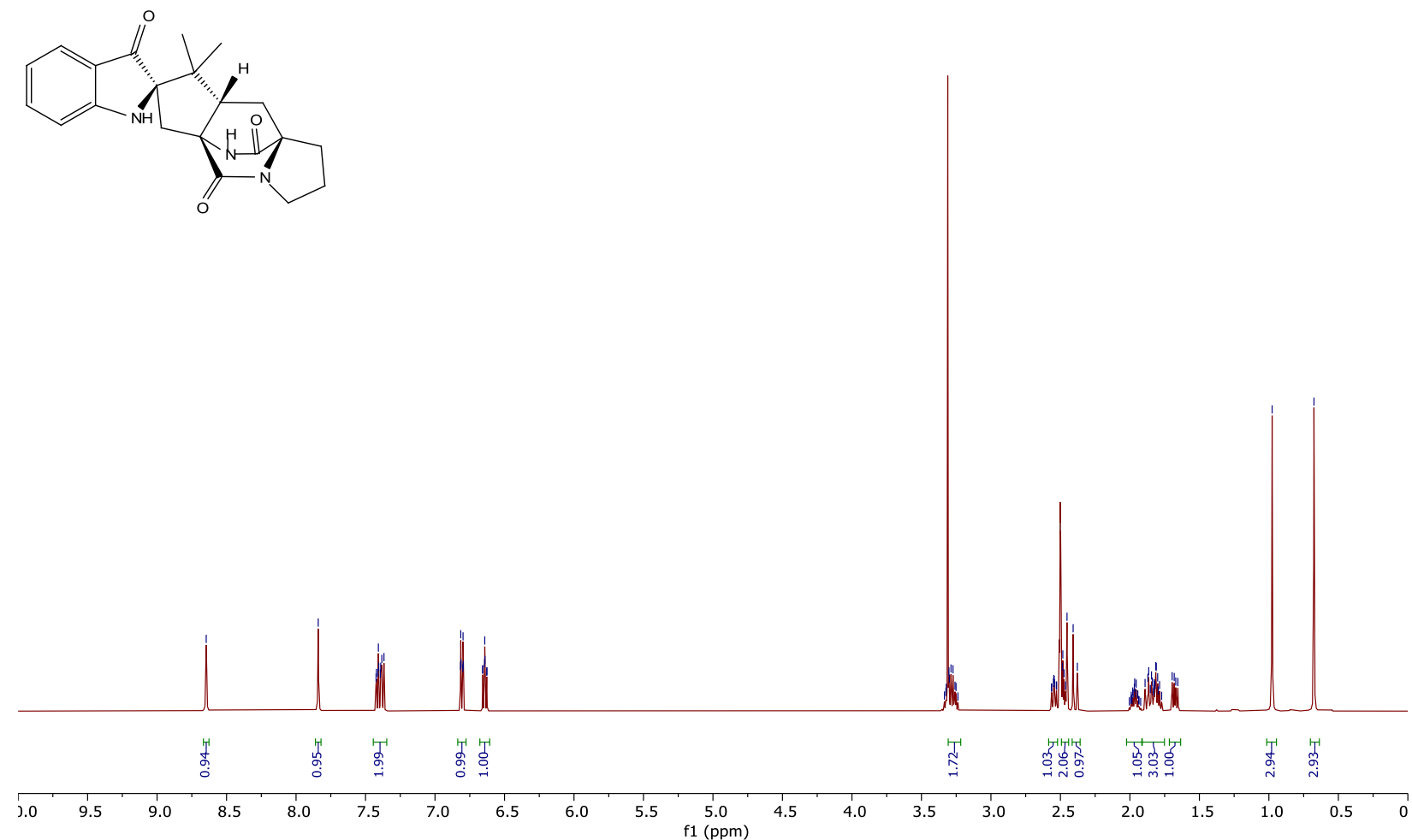

4.25 ${ }^{13} \mathrm{C}$ NMR Spectrum of Brevianamide A (1) $\left(126 \mathrm{MHz},\left(\mathrm{CD}_{3}\right)_{2} \mathrm{SO}\right)$
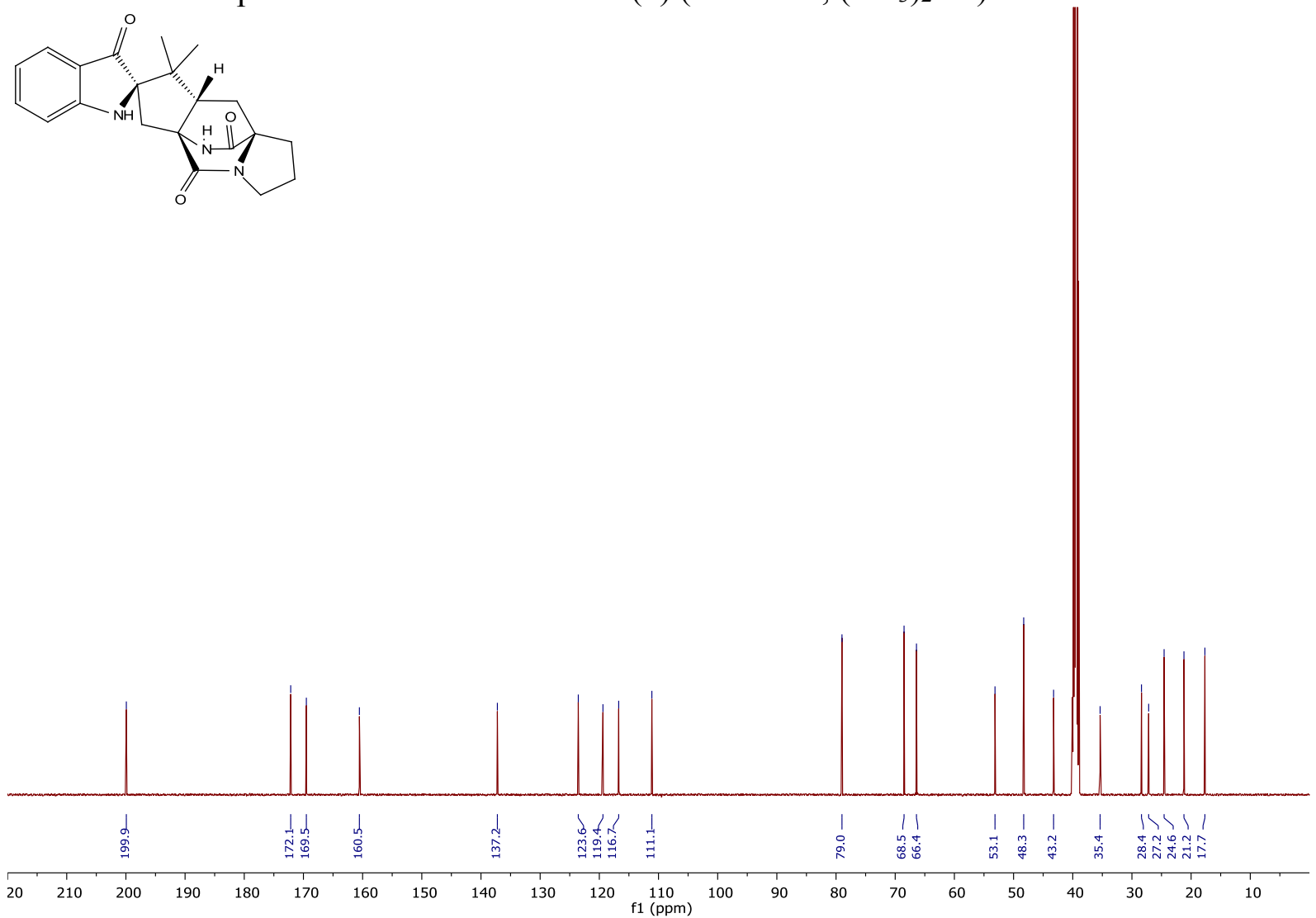
4.26 ${ }^{1} \mathrm{H}-{ }^{1} \mathrm{H}$ COSY Spectrum of Brevianamide A (1) $\left(\left(\mathrm{CD}_{3}\right)_{2} \mathrm{SO}\right)$

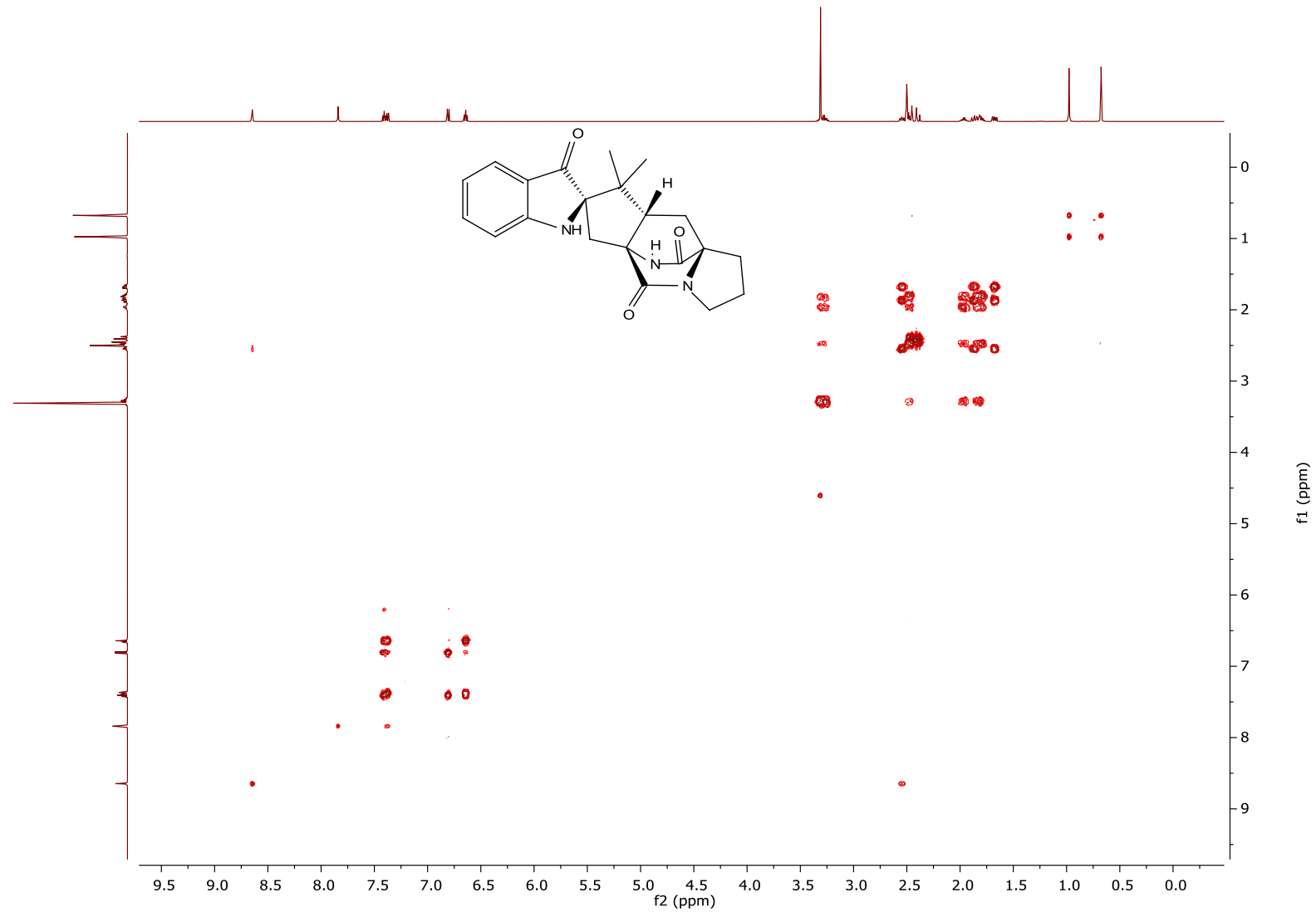

4.27 ${ }^{1} \mathrm{H}_{-}{ }^{13} \mathrm{C}$ HSQC Spectrum of Brevianamide A (1) $\left(\left(\mathrm{CD}_{3}\right)_{2} \mathrm{SO}\right)$

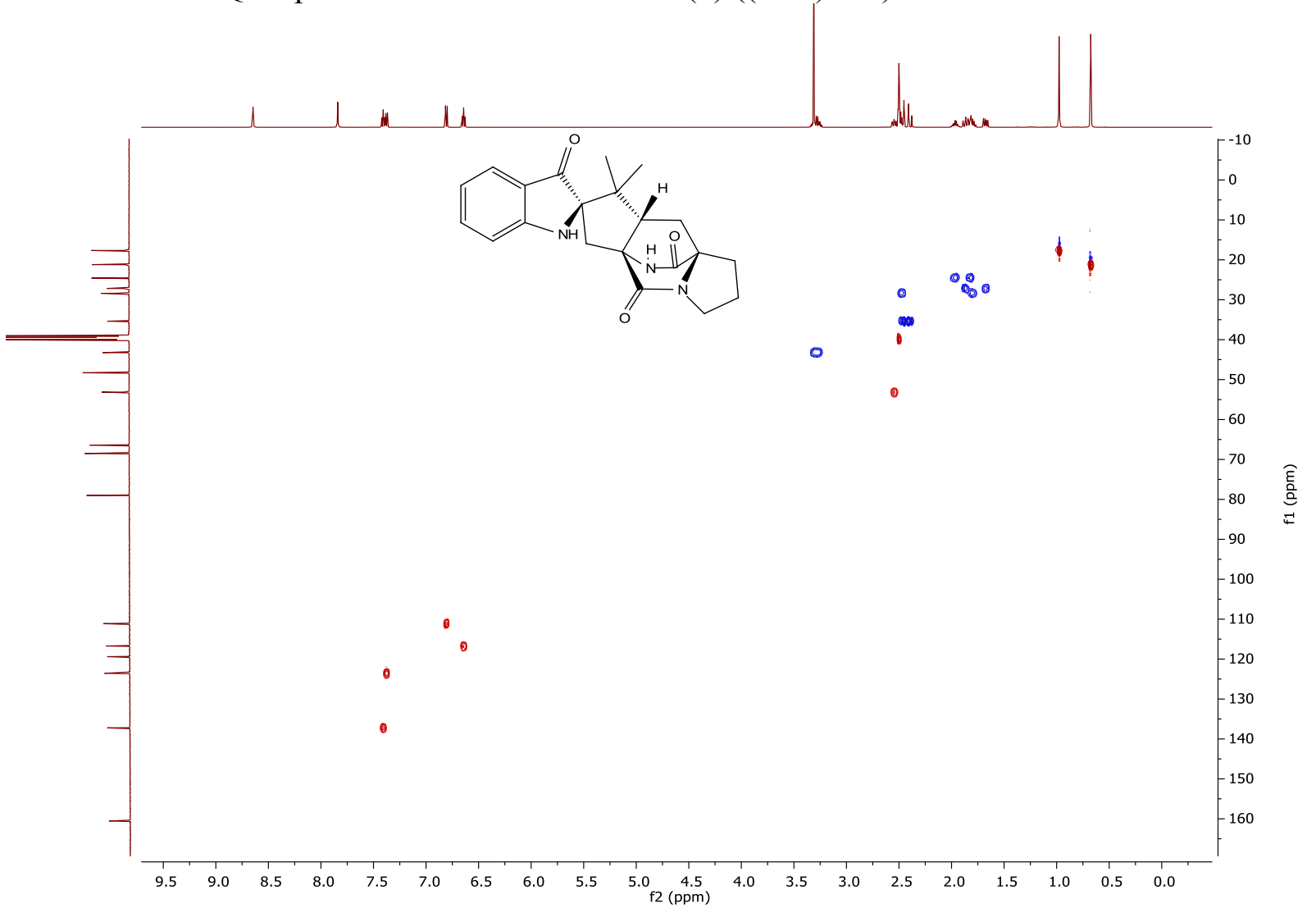


4.28 ${ }^{1} \mathrm{H}^{-13} \mathrm{C}$ HMBC Spectrum of Brevianamide A (1) $\left(\left(\mathrm{CD}_{3}\right)_{2} \mathrm{SO}\right)$
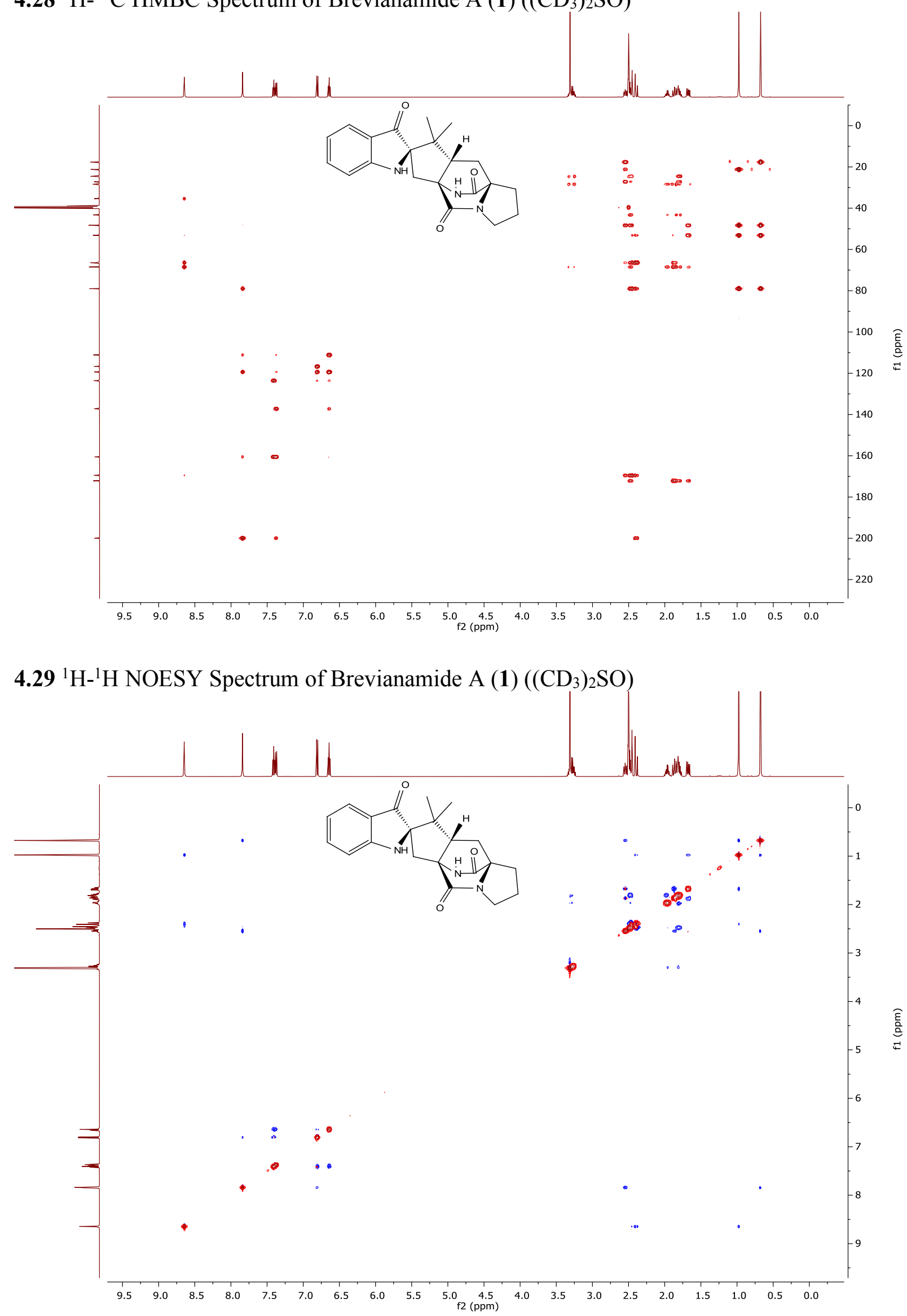
4.30 ${ }^{1} \mathrm{H}$ NMR Spectrum of Brevianamide B (2) (500 MHz, $\left.\mathrm{CDCl}_{3}\right)$

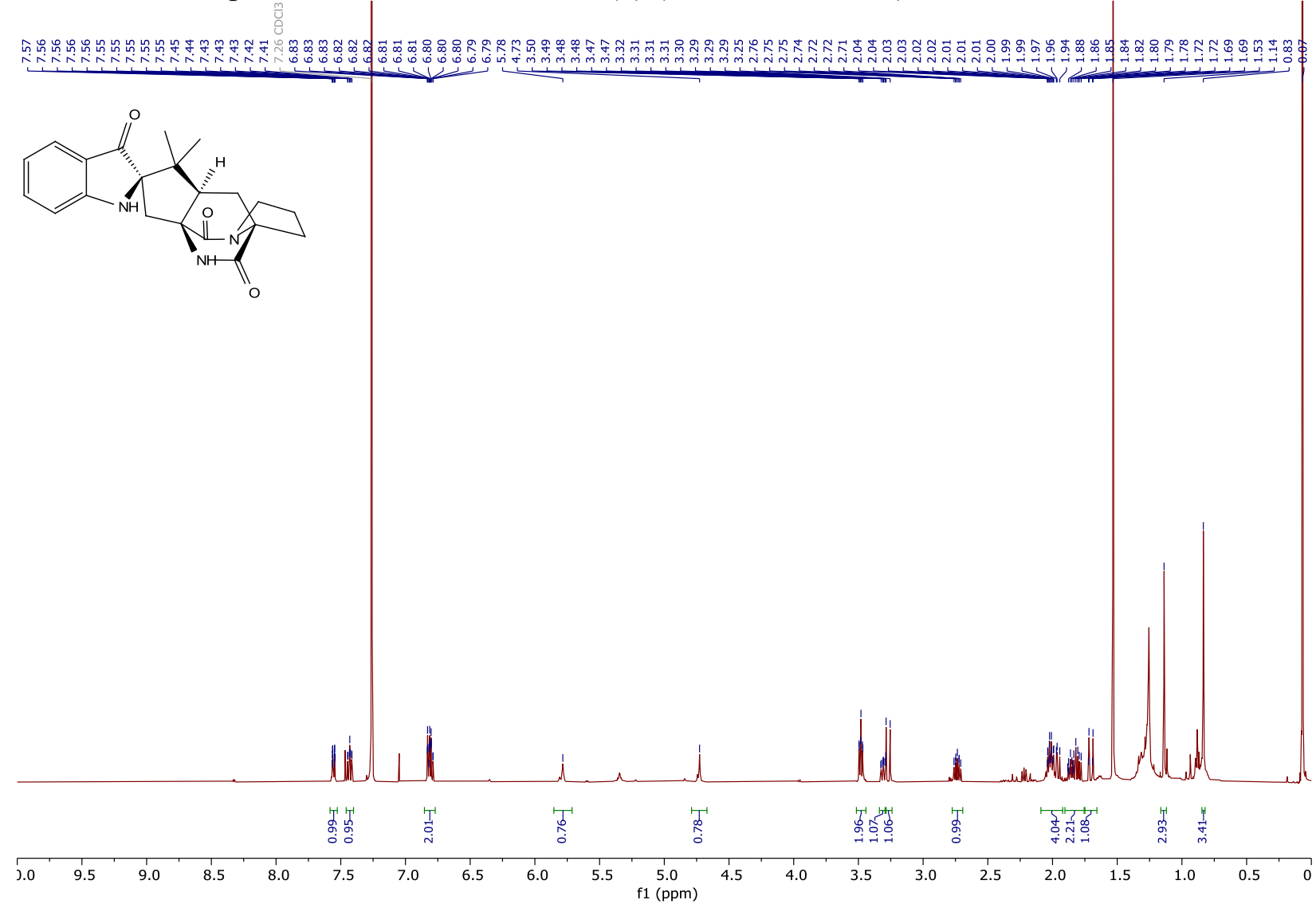

4.31 ${ }^{13} \mathrm{C}$ NMR Spectrum of Brevianamide B (2) (126 MHz, $\mathrm{CDCl}_{3}$ )

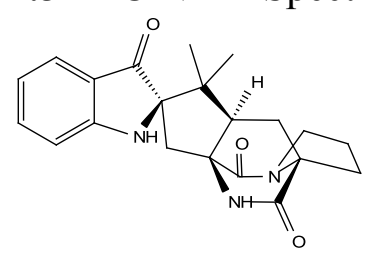

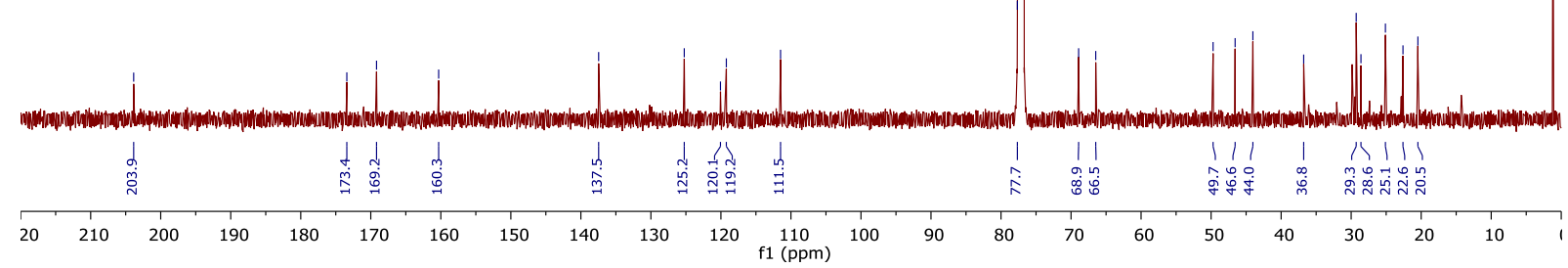


4.32 ${ }^{1} \mathrm{H}$ NMR Spectrum of Brevianamide B (2) (500 MHz, $\left.\left(\mathrm{CD}_{3}\right)_{2} \mathrm{SO}\right)$

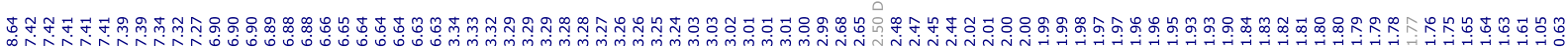
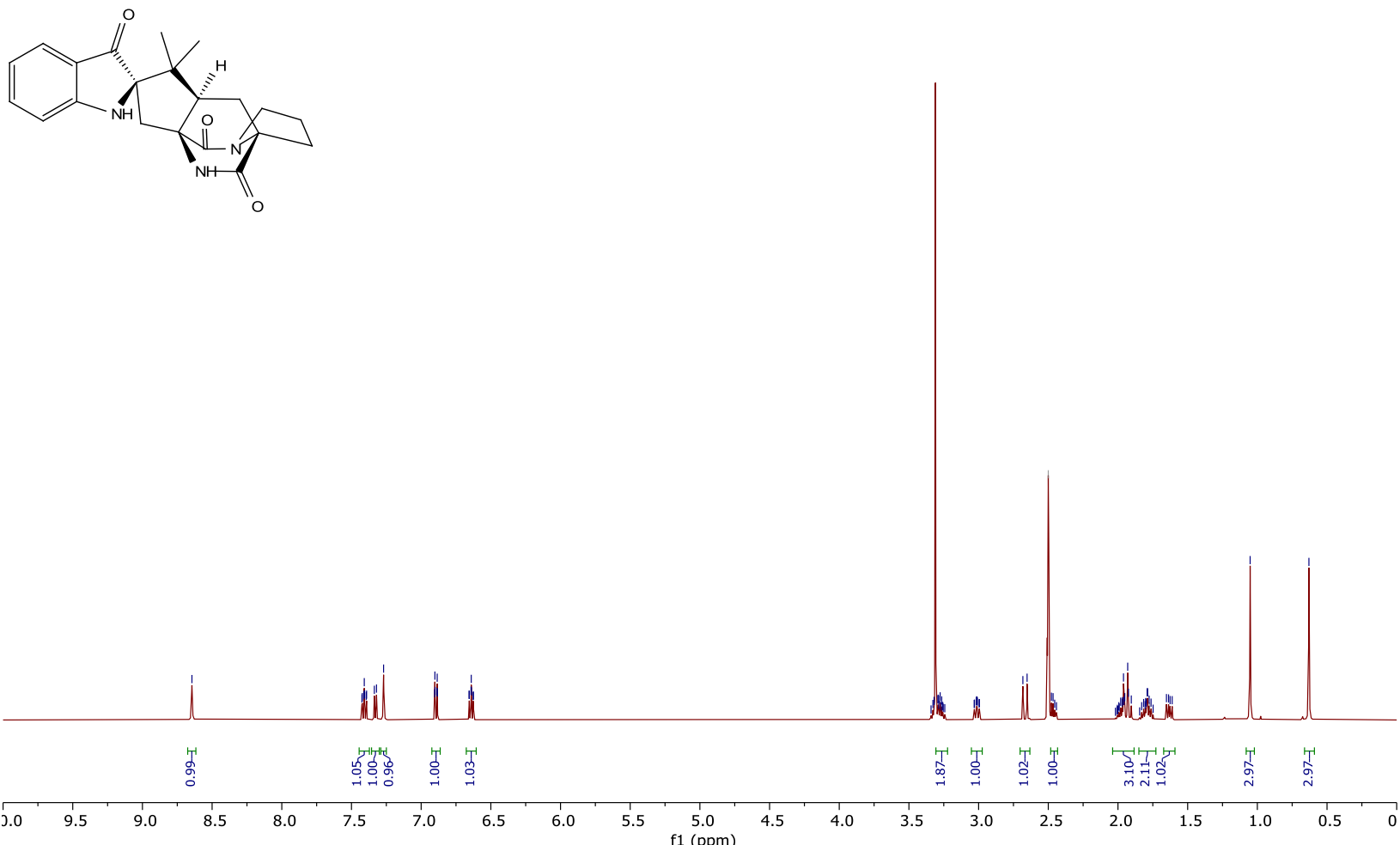

4.33 ${ }^{13} \mathrm{C}$ NMR Spectrum of Brevianamide B (2) $\left(126 \mathrm{MHz},\left(\mathrm{CD}_{3}\right)_{2} \mathrm{SO}\right)$

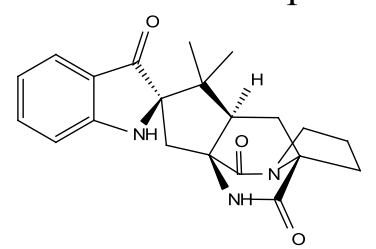

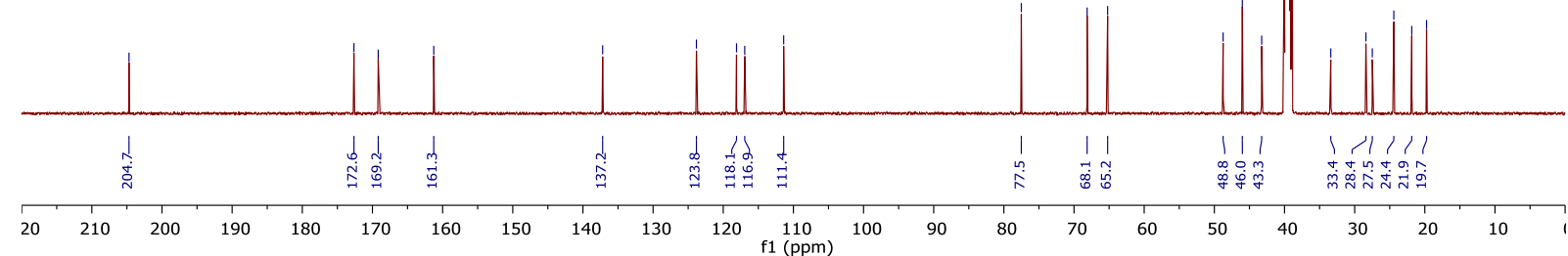


4.34 ${ }^{1} \mathrm{H}-{ }^{1} \mathrm{H}$ COSY Spectrum of Brevianamide B (2) $\left(\left(\mathrm{CD}_{3}\right)_{2} \mathrm{SO}\right)$

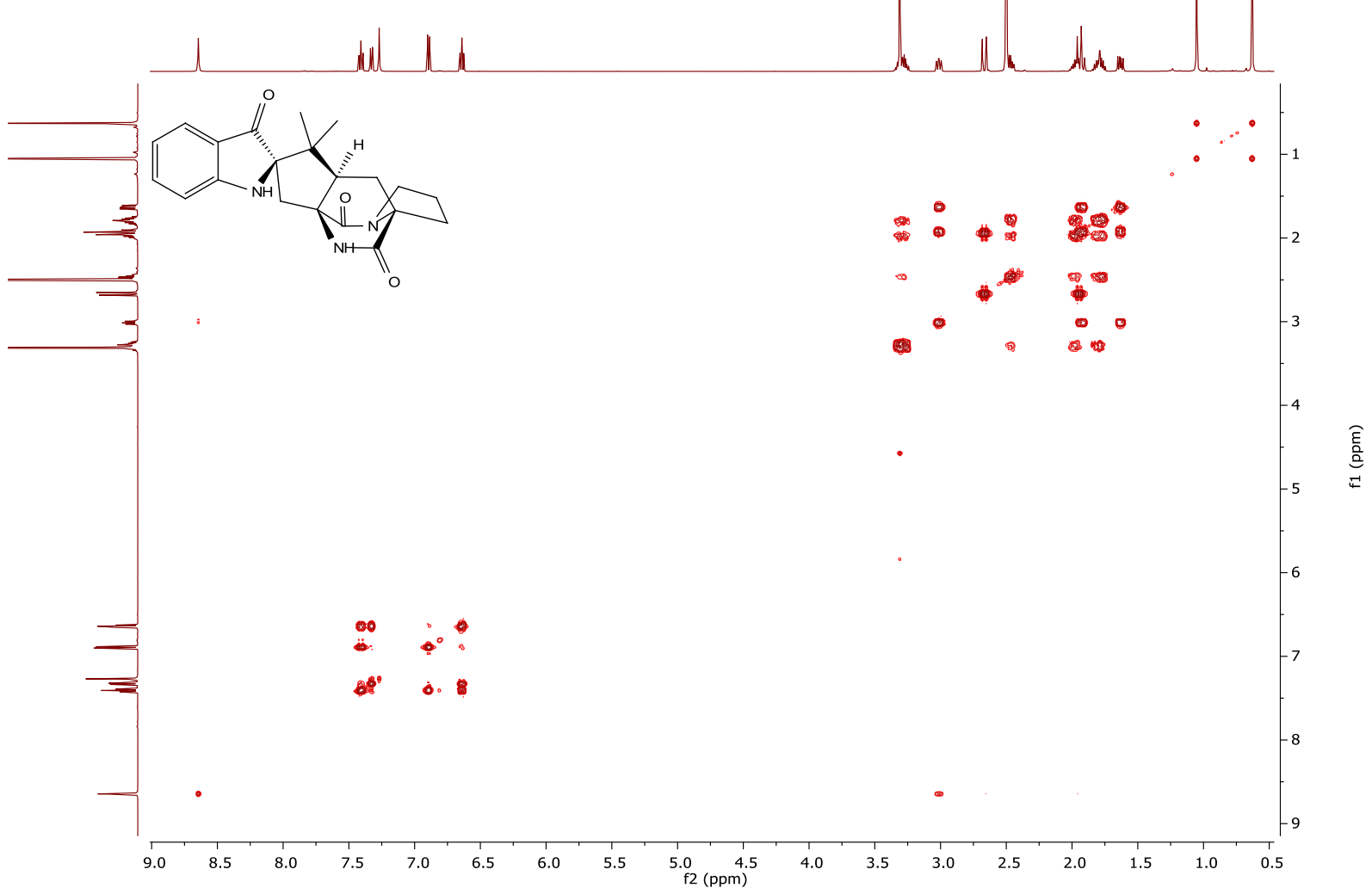

4.35 ${ }^{1} \mathrm{H}-{ }^{13} \mathrm{C}$ HSQC Spectrum of Brevianamide B (2) $\left(\left(\mathrm{CD}_{3}\right)_{2} \mathrm{SO}\right)$

48 
$4.36{ }^{1} \mathrm{H}-{ }^{13} \mathrm{C}$ HMBC Spectrum of Brevianamide B (2) $\left(\left(\mathrm{CD}_{3}\right)_{2} \mathrm{SO}\right)$

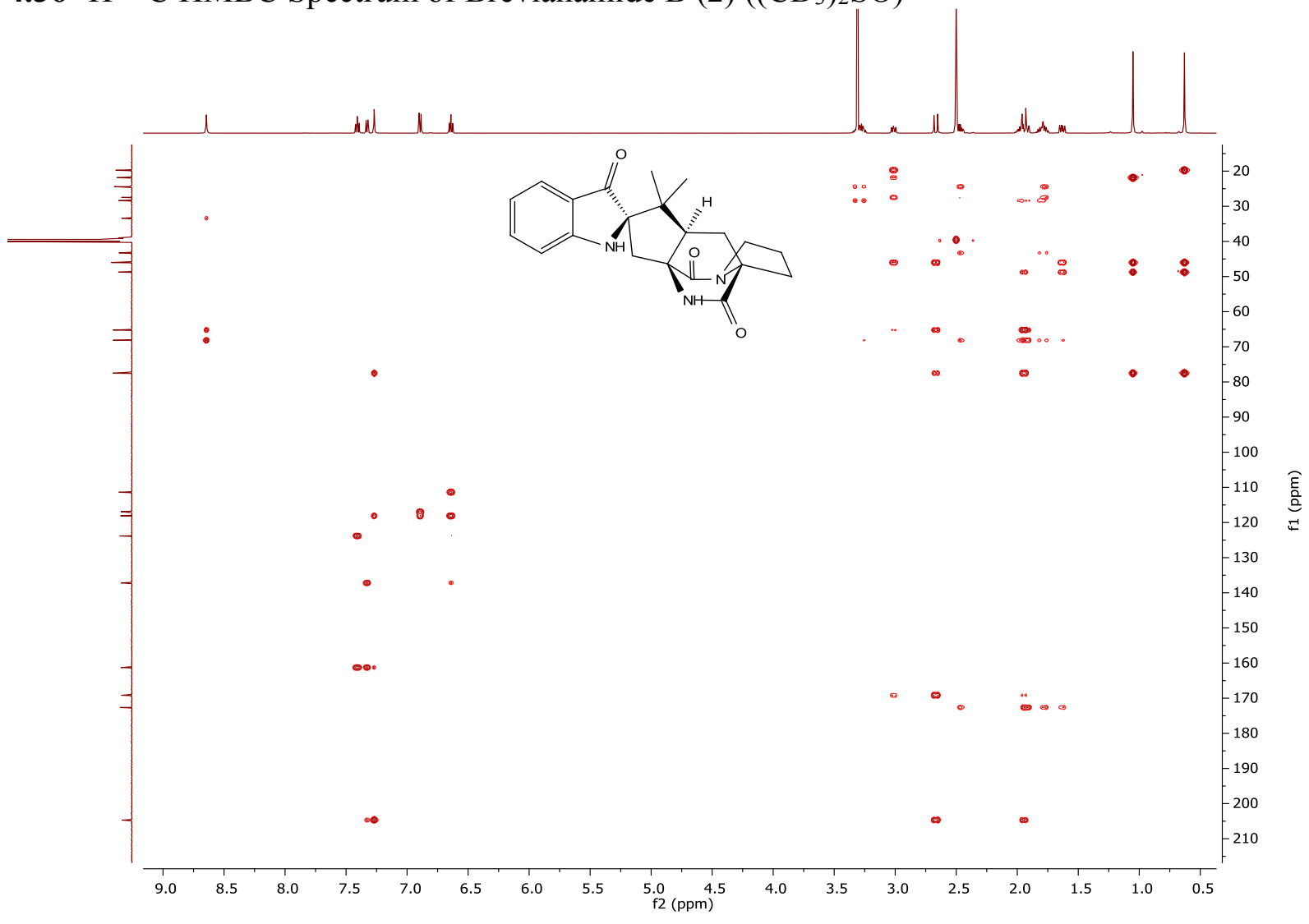

4.37 ${ }^{1} \mathrm{H}-{ }^{1} \mathrm{H}$ NOESY Spectrum of Brevianamide B (2) $\left(\left(\mathrm{CD}_{3}\right)_{2} \mathrm{SO}\right)$

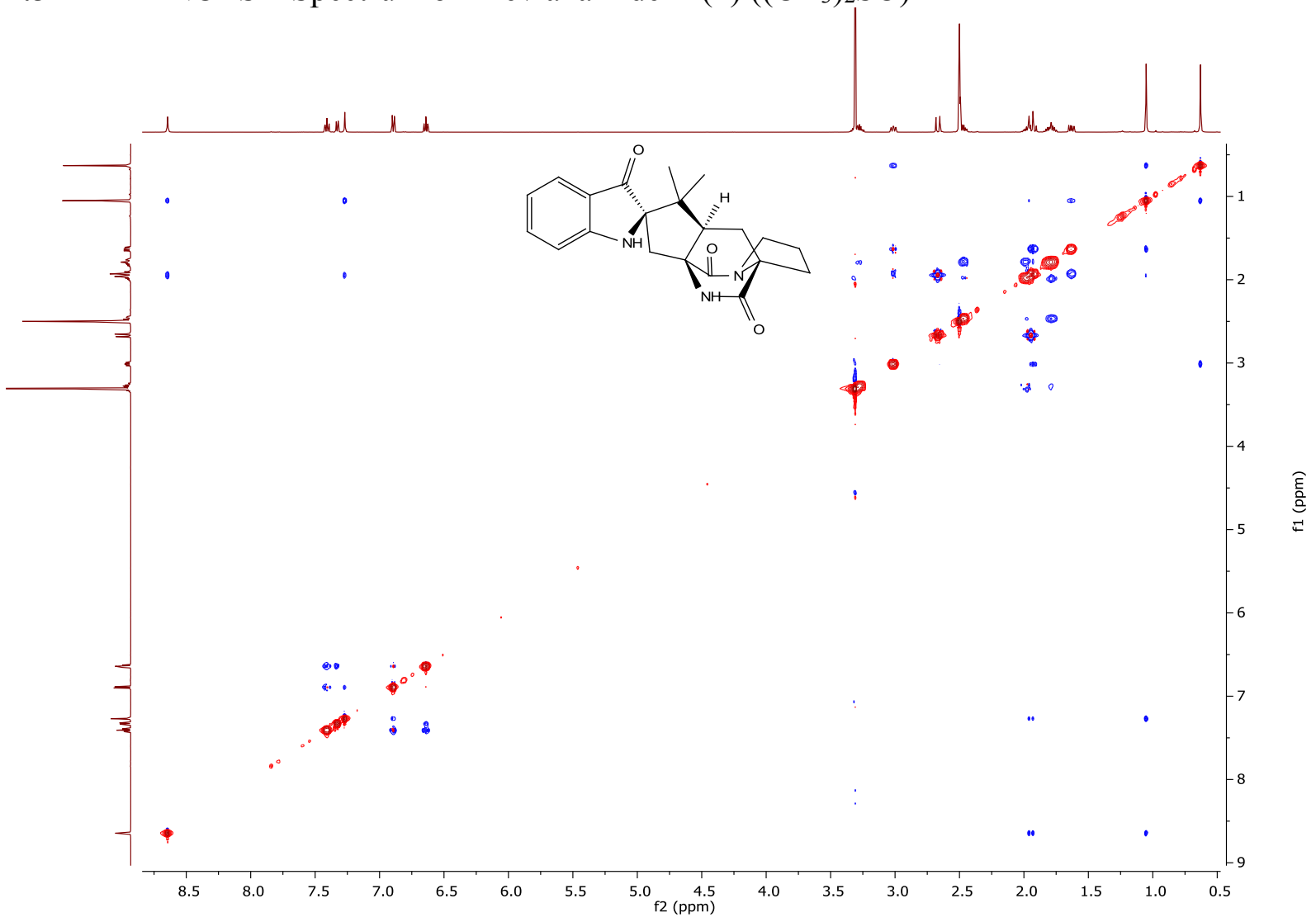


4.38 ${ }^{1} \mathrm{H}$ NMR Spectrum of Compound $18\left(500 \mathrm{MHz}, \mathrm{CDCl}_{3}\right)$
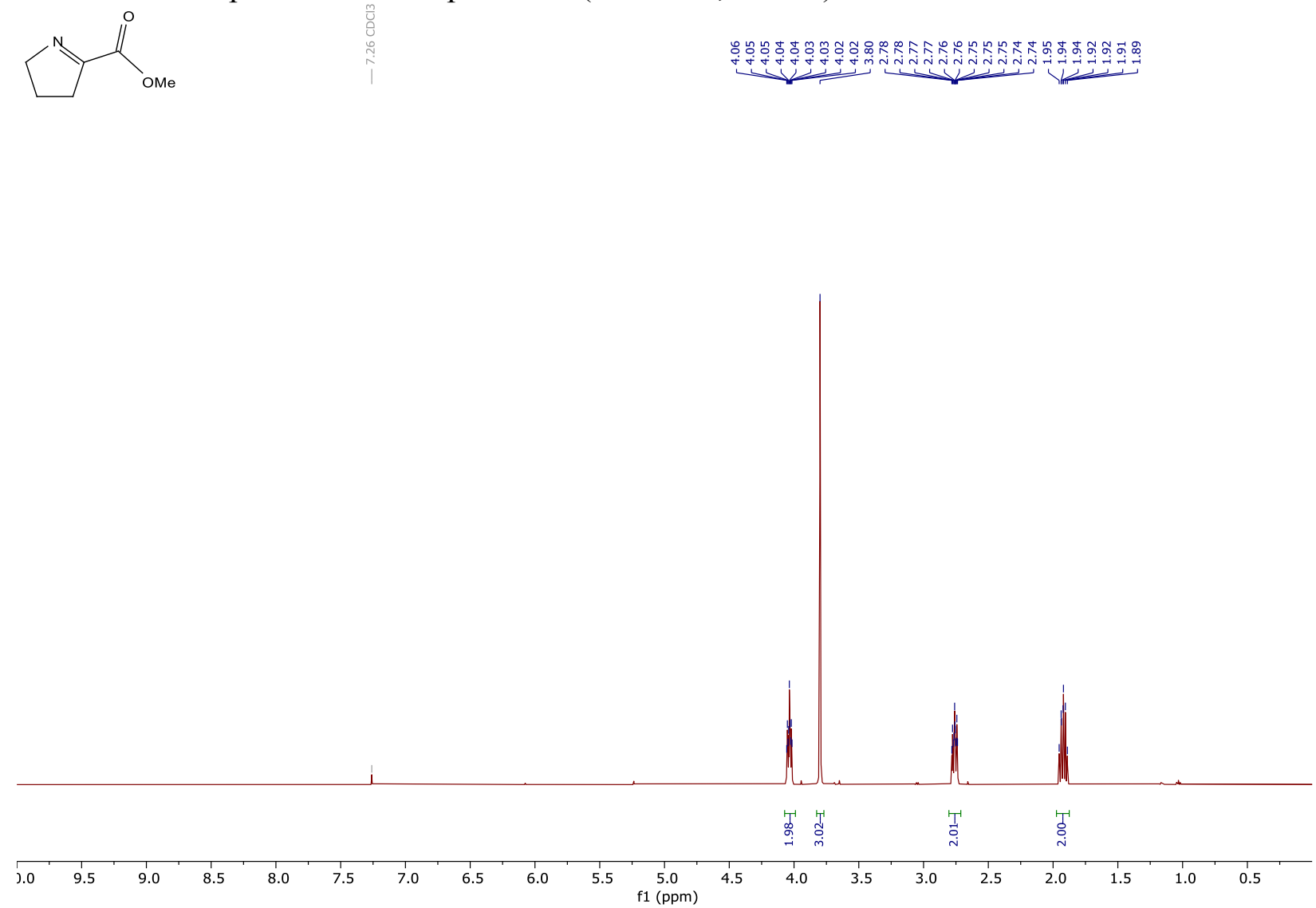

4.39 ${ }^{13} \mathrm{C}$ NMR Spectrum of Compound $18\left(126 \mathrm{MHz}, \mathrm{CDCl}_{3}\right)$
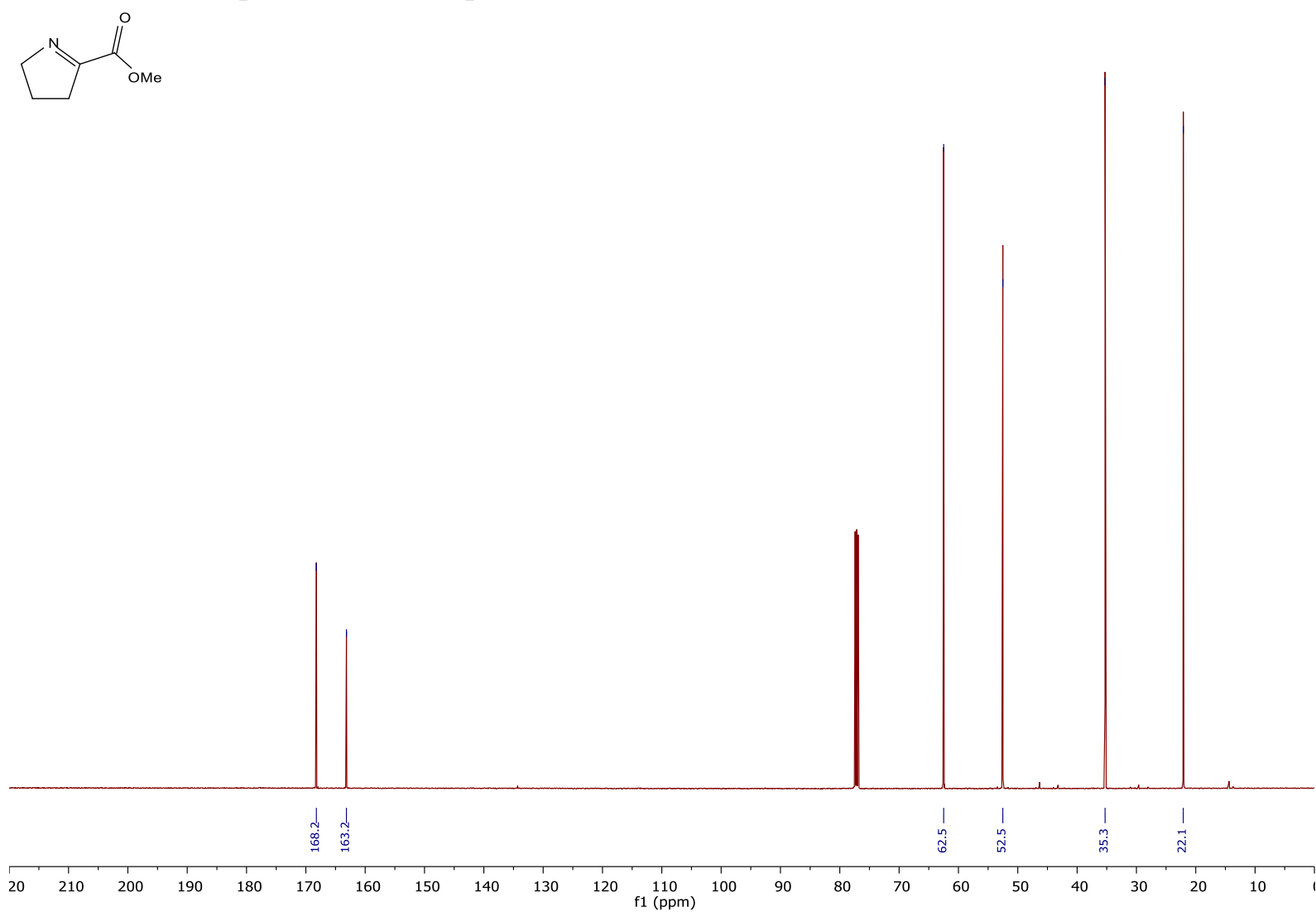
4.40 ${ }^{1} \mathrm{H}$ NMR Spectrum of 3-Chloro-3-methyl-1-butyne (500 MHz, $\mathrm{CDCl}_{3}$ )<smiles>C=CC(C)(C)Cl</smiles>

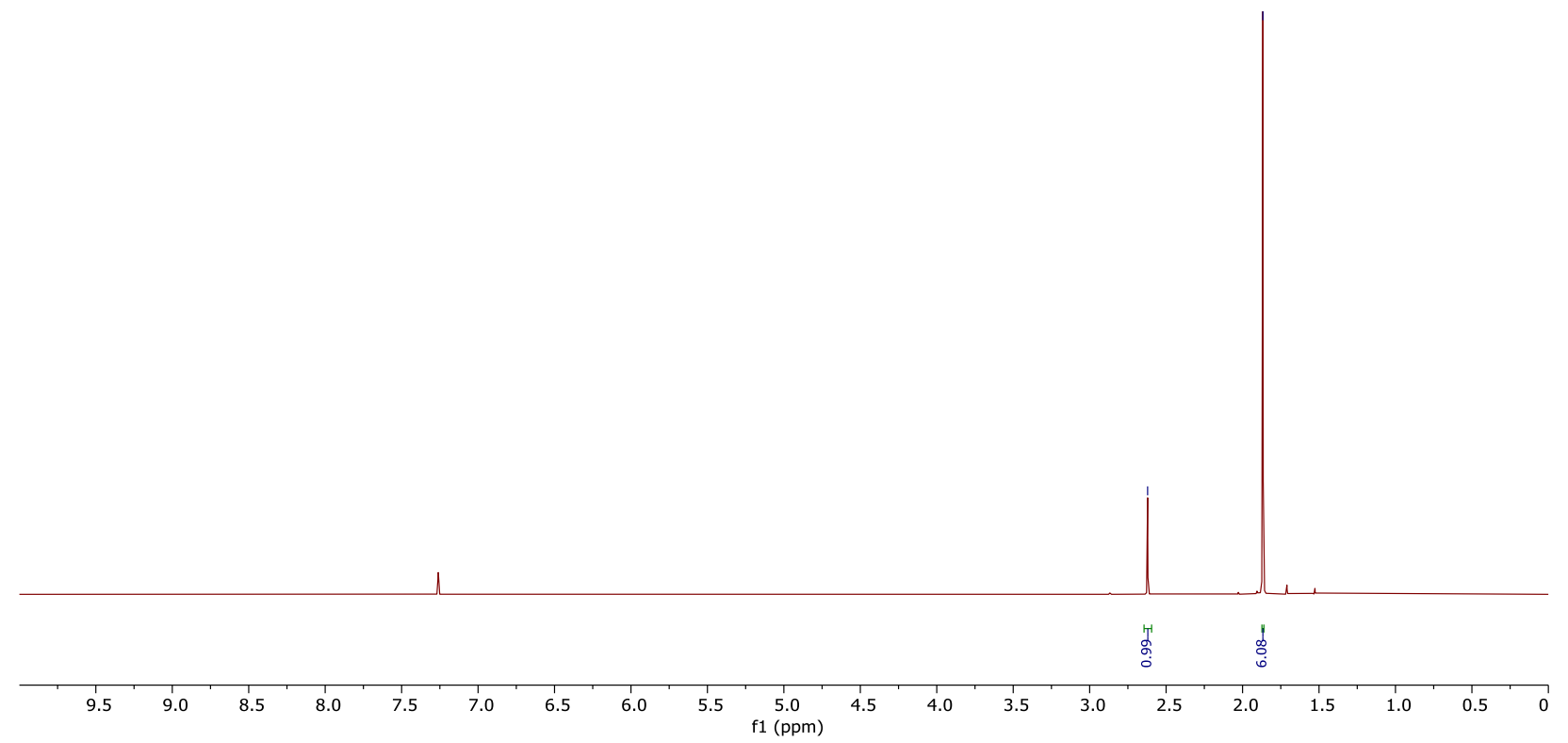

4.41 ${ }^{13} \mathrm{C}$ NMR Spectrum of 3-Chloro-3-methyl-1-butyne (101 MHz, $\mathrm{CDCl}_{3}$ )
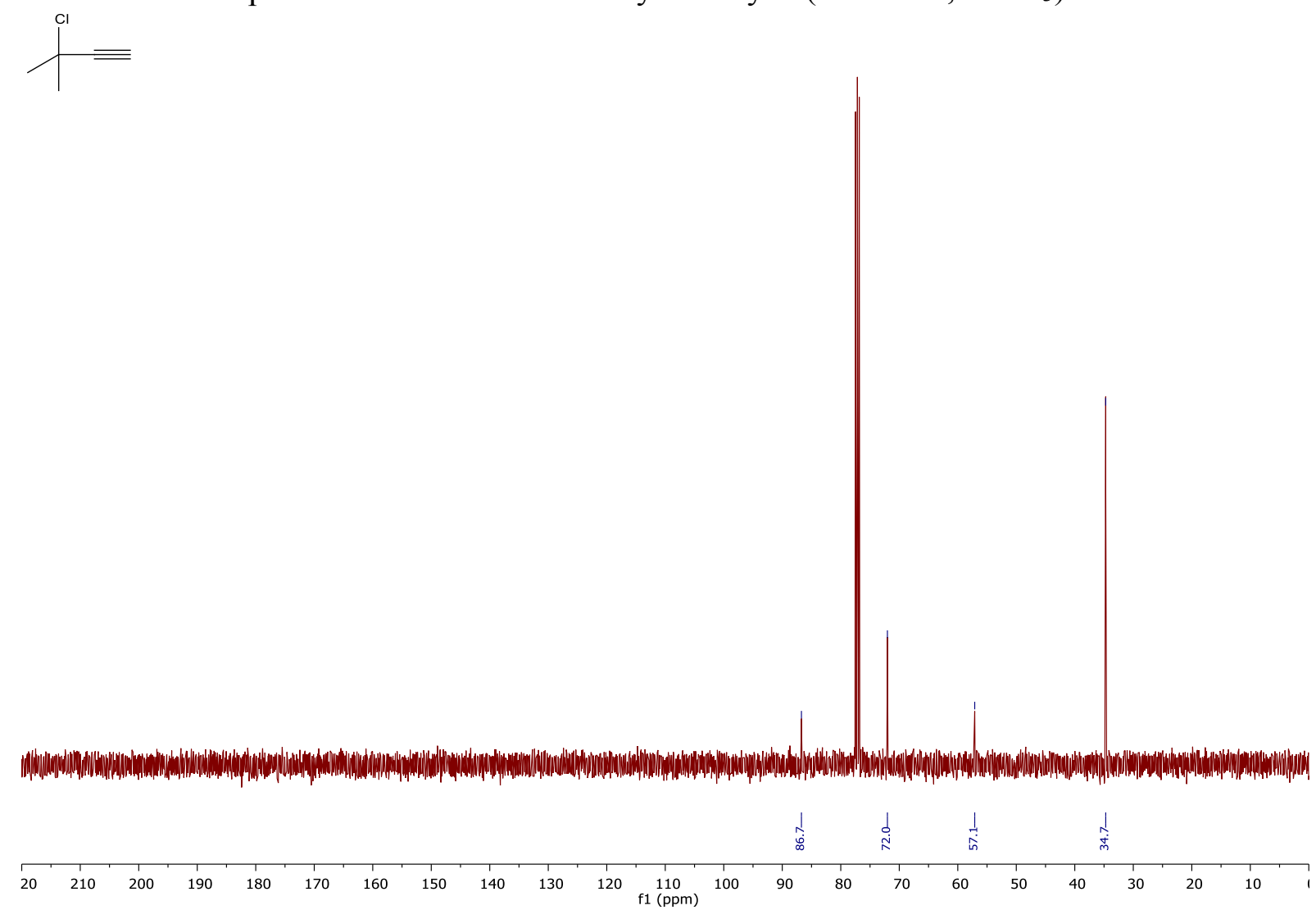
4.42 ${ }^{1} \mathrm{H}$ NMR Spectrum of 1,1-Dimethylallene (500 MHz, $\mathrm{CDCl}_{3}$ )
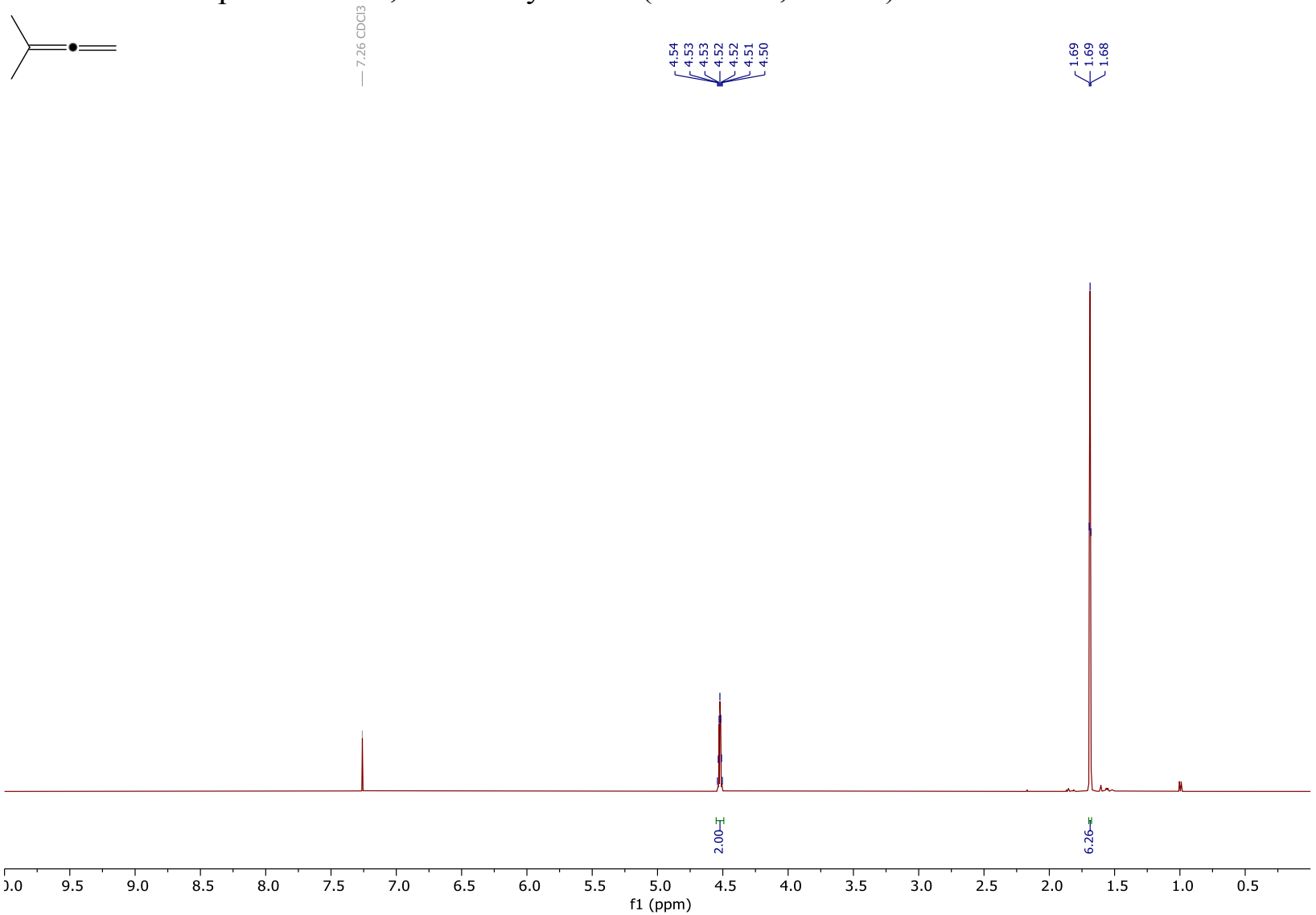

4.43 ${ }^{13} \mathrm{C}$ NMR Spectrum of 1,1-Dimethylallene (151 $\mathrm{MHz}, \mathrm{CDCl}_{3}$ )
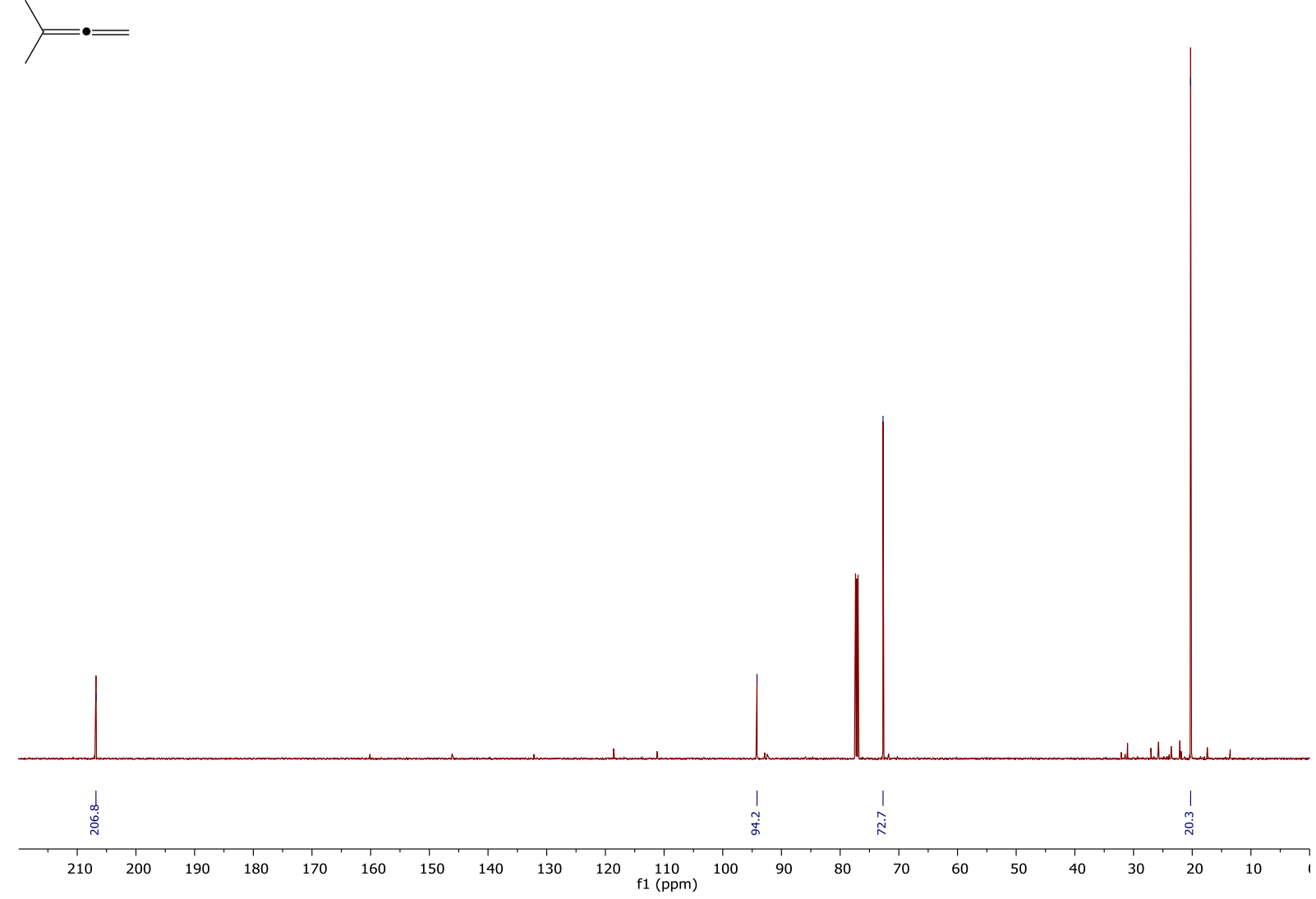
4.44 ${ }^{1} \mathrm{H}$ NMR Spectrum of $t$ - $\mathrm{BuOCl}\left(500 \mathrm{MHz}, \mathrm{CDCl}_{3}\right)$

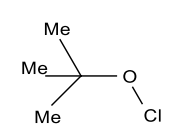

$\stackrel{m}{\oplus}$

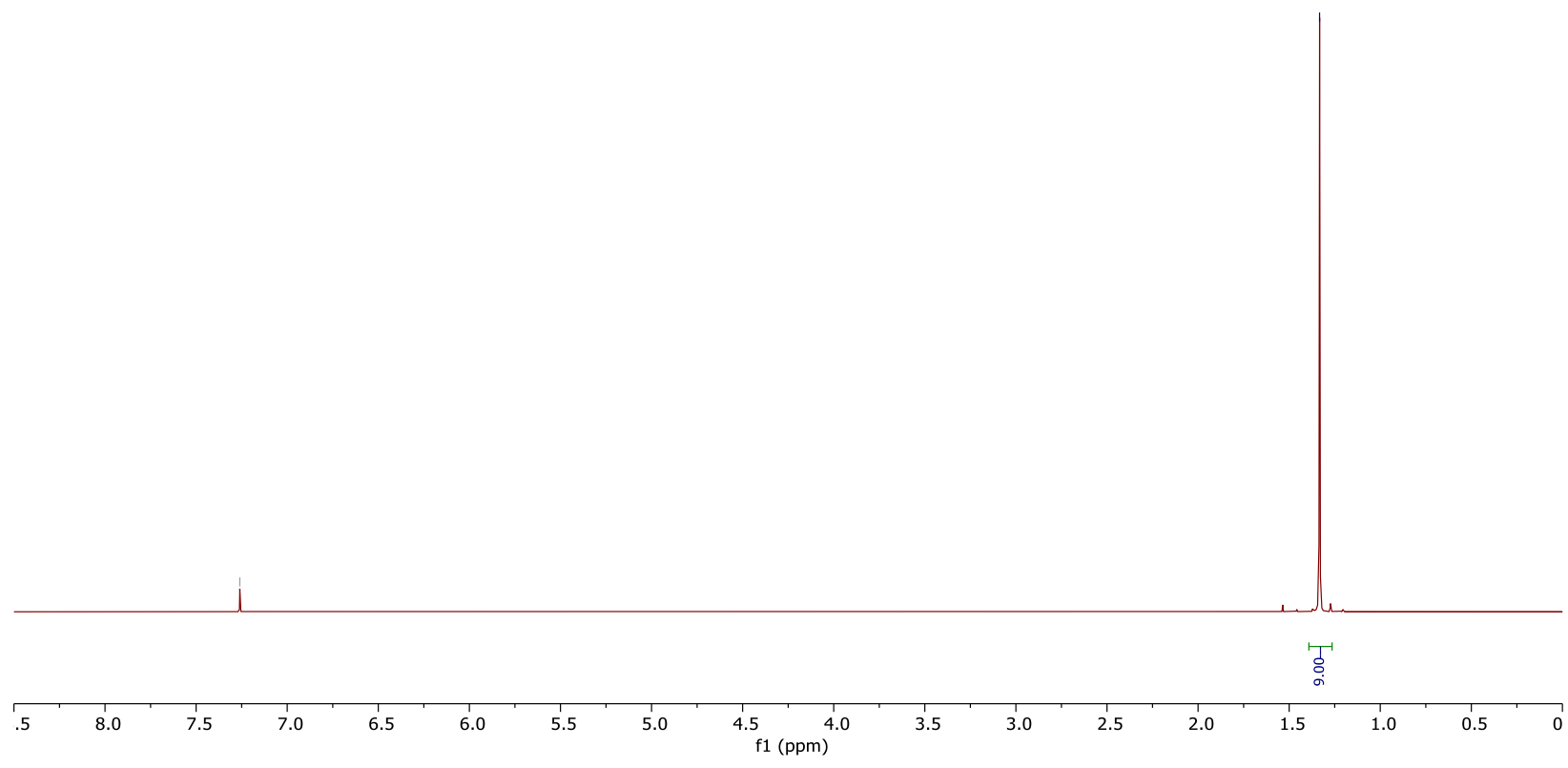

4.45 ${ }^{13} \mathrm{C}$ NMR Spectrum of $t$ - $\mathrm{BuOCl}\left(126 \mathrm{MHz}, \mathrm{CDCl}_{3}\right)$
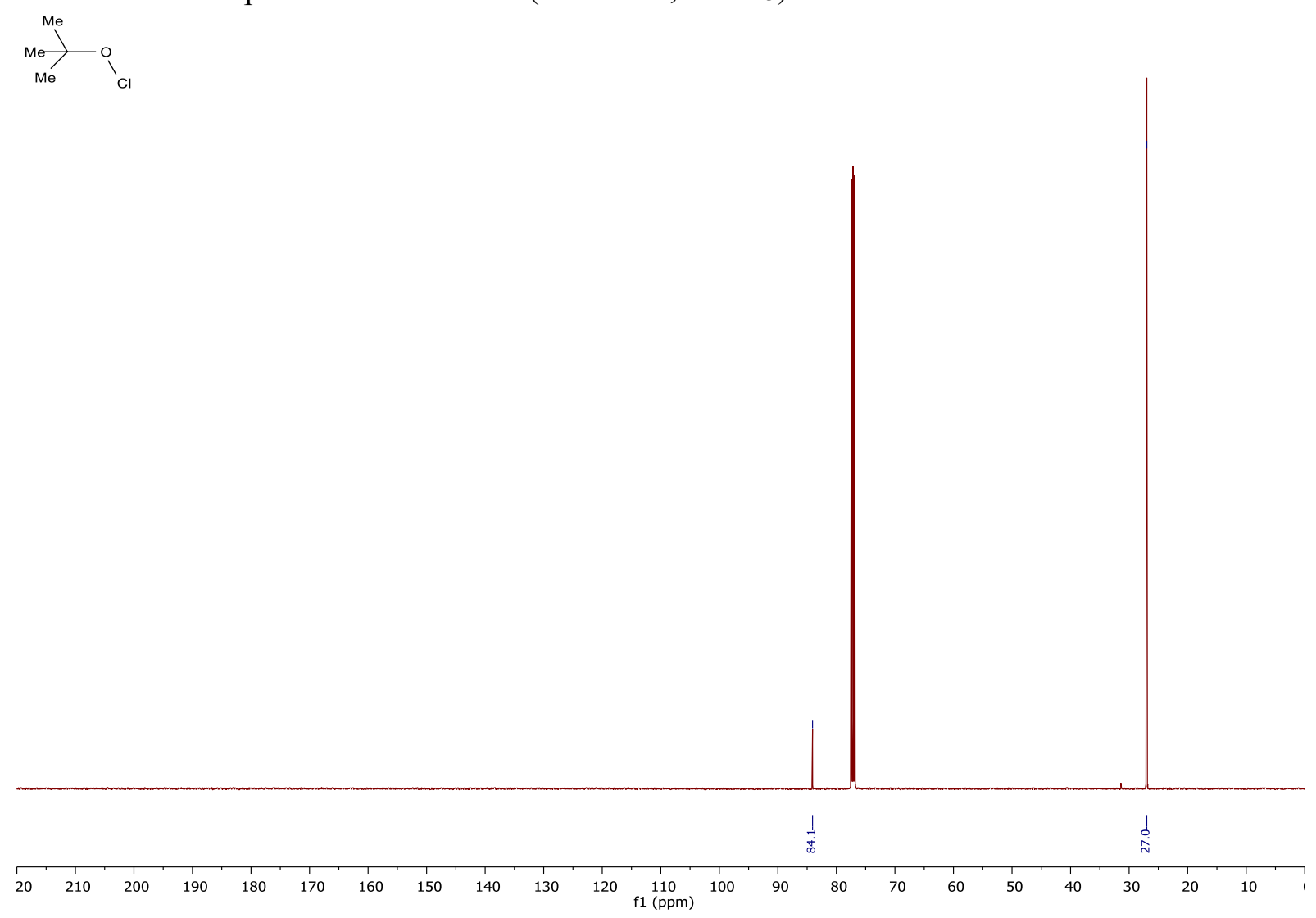

53 


\section{Chiral HPLC Chromatograms}

5.1 Chiral HPLC Chromatograms of Brevianamide A

$(+) /(-)$-brevianamide A (1)

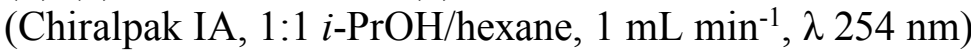

$\mathrm{t}_{\mathrm{R} 1}=5.40 \mathrm{~min}, \mathrm{t}_{\mathrm{R} 2}=6.74 \mathrm{~min}$.

\section{$<$ Chromatogram $>$}

$\mathrm{mV}$

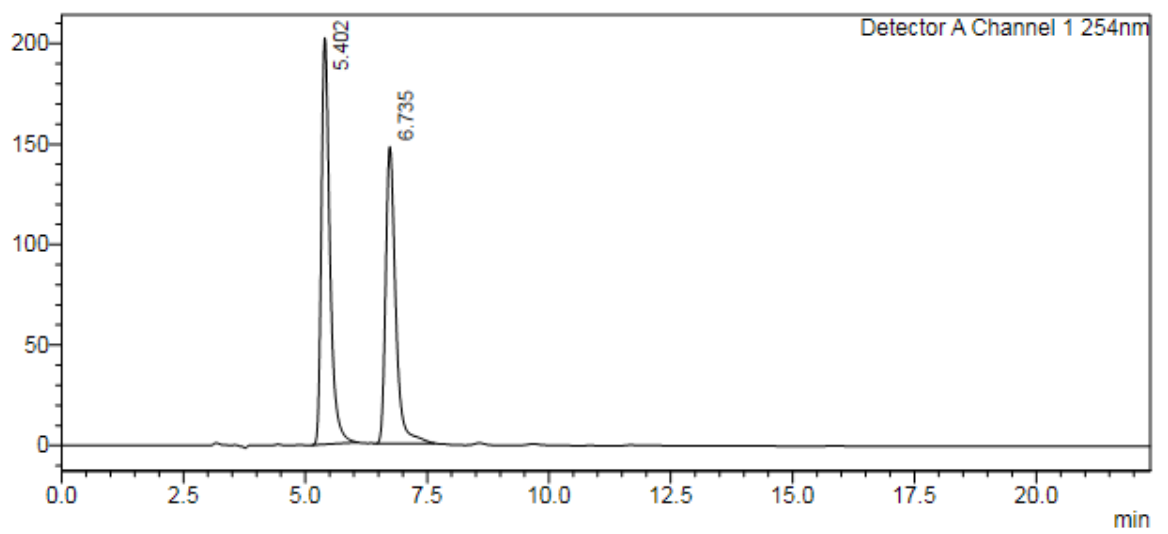

<Peak Table>

Detector A Channel $1254 \mathrm{~nm}$

\begin{tabular}{|c|c|c|c|c|c|c|c|}
\hline $\begin{array}{r}\text { Peak\# } \\
1\end{array}$ & $\begin{array}{r}\text { Ret. Time } \\
5.402\end{array}$ & $\begin{array}{l}\text { Area } \\
2456350\end{array}$ & $\begin{array}{l}\text { Height } \\
202003\end{array}$ & $\begin{array}{l}\text { Conc. } \\
53.925\end{array}$ & Unit & Mark & Name \\
\hline $\begin{array}{r}2 \\
\text { Total }\end{array}$ & 6.735 & $\begin{array}{l}2098773 \\
4555123\end{array}$ & $\begin{array}{l}147492 \\
349495\end{array}$ & 46.075 & & & \\
\hline
\end{tabular}

(+)-brevianamide A (1) e.r. 93:7

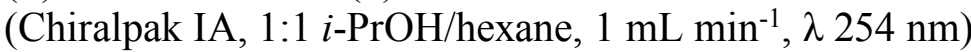

$\mathrm{t}_{\text {Rminor }}=5.41 \mathrm{~min}, \mathrm{t}_{\text {Rmajor }}=6.72 \mathrm{~min}$.

$<$ Chromatogram $>$

$\mathrm{mV}$

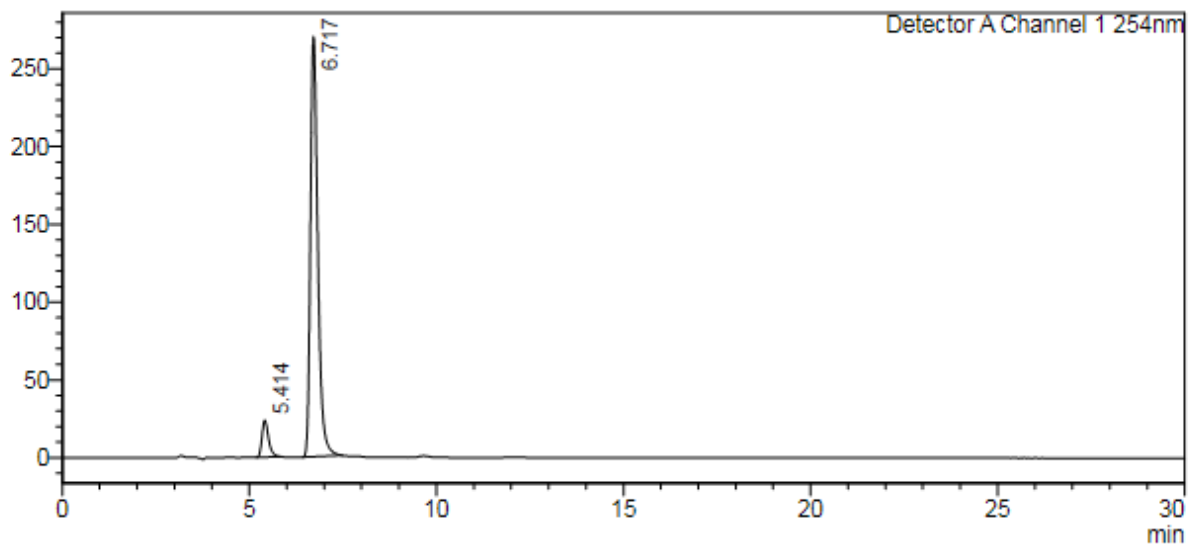

<Peak Table>

Detector A Channel $1254 \mathrm{~nm}$

\begin{tabular}{|c|c|c|c|c|c|c|c|}
\hline $\begin{array}{r}\text { Peak\# } \\
1\end{array}$ & $\begin{array}{r}\text { Ret. Time } \\
5.414\end{array}$ & $\begin{array}{l}\text { Area } \\
289622\end{array}$ & $\begin{array}{l}\text { Height } \\
23737\end{array}$ & $\begin{array}{l}\text { Conc. } \\
7.232\end{array}$ & Unit & Mark & Name \\
\hline 2 & 6.717 & 3715018 & 269840 & 92.768 & & & \\
\hline Total & & 4004641 & 293577 & & & & \\
\hline
\end{tabular}


(+)-brevianamide A (1) e.r. 99:1 after crystallisation

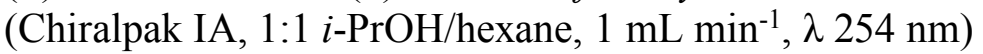

$\mathrm{t}_{\text {Rminor }}=5.51 \mathrm{~min}, \mathrm{t}_{\text {Rmajor }}=6.84 \mathrm{~min}$.

<Chromatogram>

$\mathrm{mV}$

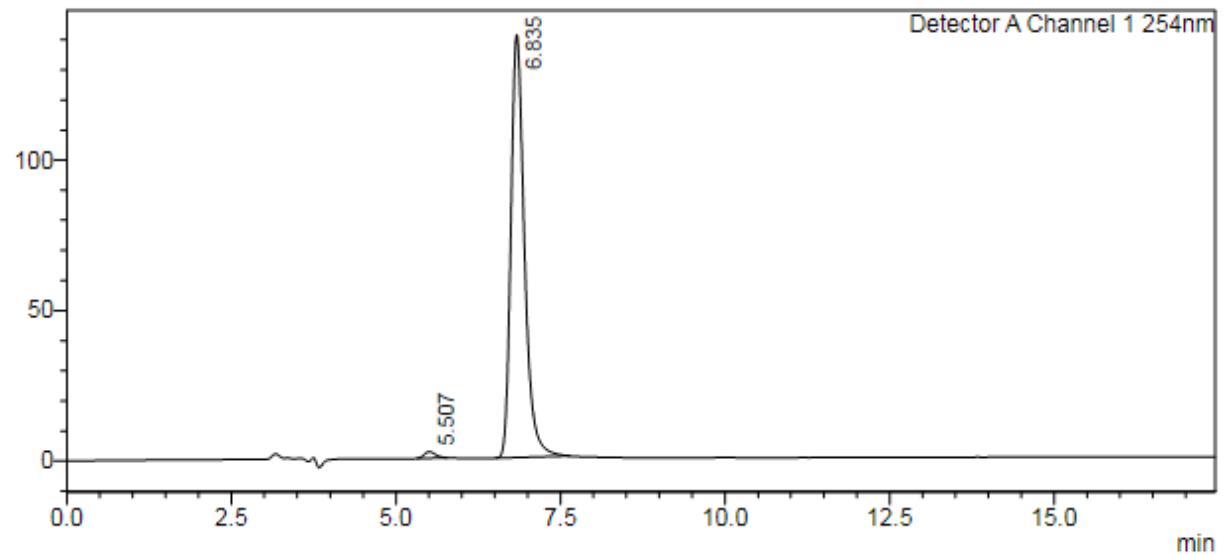

<Peak Table>

\begin{tabular}{|c|c|c|c|c|c|c|c|}
\hline $\begin{array}{r}\text { Detecto } \\
\text { Peak\# } \\
1\end{array}$ & $\begin{array}{r}\text { or A Channe } \\
\text { Ret. Time } \\
5.507\end{array}$ & $\begin{array}{l}254 \mathrm{~nm} \\
\text { Area } \\
24842\end{array}$ & $\begin{array}{l}\text { Height } \\
2113\end{array}$ & $\begin{array}{l}\text { Conc. } \\
1.220\end{array}$ & Unit & Mark & Name \\
\hline $\begin{array}{c}2 \\
\text { Total }\end{array}$ & 6.835 & $\begin{array}{l}2010703 \\
2035545\end{array}$ & $\begin{array}{l}140355 \\
142469\end{array}$ & 98.780 & & & \\
\hline
\end{tabular}

(-)-brevianamide A (1) e.r. 95:5

(Chiralpak IA, 1:1 $i$-PrOH/hexane, $1 \mathrm{~mL} \mathrm{~min}^{-1}, \lambda 254 \mathrm{~nm}$ )

$t_{\text {Rmajor }}=5.38 \mathrm{~min}, \mathrm{t}_{\mathrm{Rminor}}=6.74 \mathrm{~min}$.

\section{<Chromatogram>}

$\mathrm{mV}$

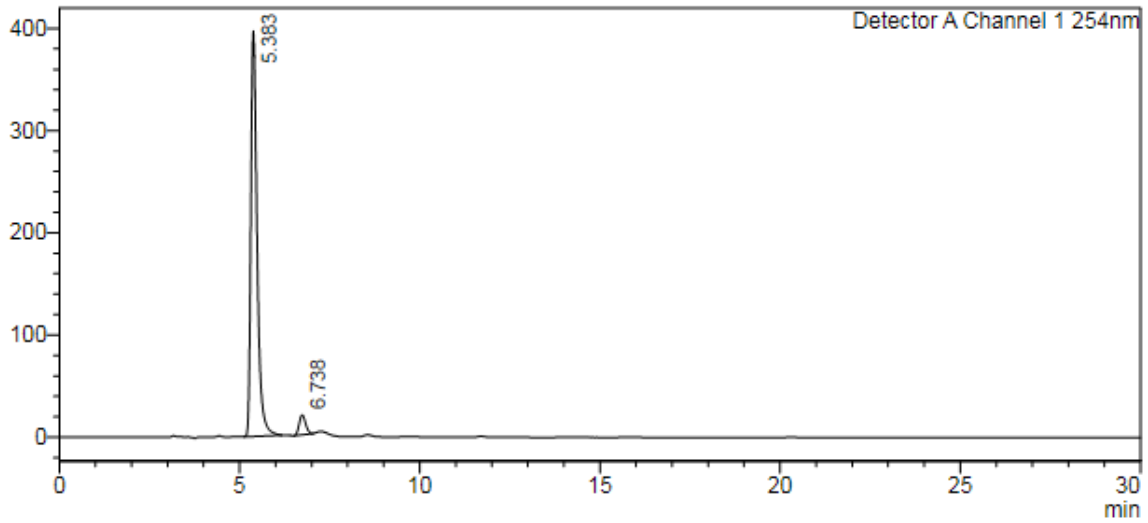

\section{<Peak Table>}

Detector A Channel $1254 \mathrm{~nm}$

Peak\# Ret. Time Area

\begin{tabular}{|r|r|r|r|r|r|r|r|}
\hline 1 & 5.383 & 4758681 & \multicolumn{1}{|c|}{ Height } & Conc. & Unit & Mark & Name \\
\hline 2 & 6.738 & 241511 & 19400 & 95.170 & & & \\
Total & & 5000192 & 415507 & & & & \\
\hline
\end{tabular}


5.2 Chiral HPLC Chromatograms of Brevianamide B

$(+) /(-)$-Brevianamide B (2)

(Chiralpak IA, 1:3 EtOH/hexane, $1 \mathrm{~mL} \min ^{-1}, \lambda 254 \mathrm{~nm}$ )

$\mathrm{t}_{\mathrm{R} 1}=13.86 \mathrm{~min}, \mathrm{t}_{\mathrm{R} 2}=26.03 \mathrm{~min}$.

\section{$<$ Chromatogram>}

$\mathrm{mV}$

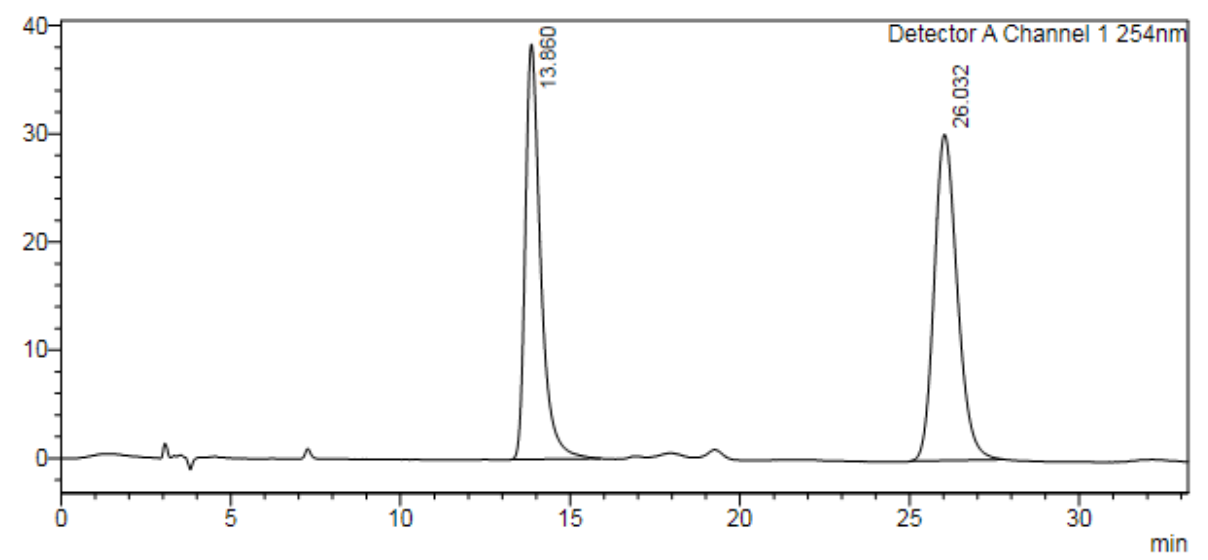

<Peak Table>

Detector A Channel $1254 \mathrm{~nm}$

\begin{tabular}{|c|c|c|c|c|c|c|c|}
\hline $\begin{array}{r}\text { Peak\# } \\
1\end{array}$ & $\begin{array}{r}\text { Ret. Time } \\
13.860\end{array}$ & $\begin{array}{l}\text { Area } \\
1202069\end{array}$ & $\begin{array}{l}\text { Height } \\
38310\end{array}$ & $\begin{array}{l}\text { Conc. } \\
45.782\end{array}$ & Unit & Mark & Name \\
\hline $\begin{array}{r}2 \\
\text { Total }\end{array}$ & 26.032 & $\begin{array}{l}1423551 \\
2625620\end{array}$ & $\begin{array}{l}30114 \\
68424\end{array}$ & 54.218 & & & \\
\hline
\end{tabular}

(+)-Brevianamide B (2) e.r. 93:7

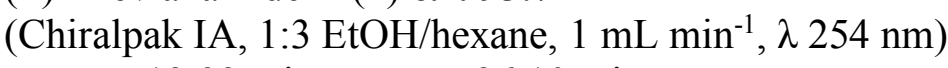

$t_{\text {Rminor }}=13.99 \mathrm{~min}, t_{\text {Rmajor }}=26.10 \mathrm{~min}$.

\section{$<$ Chromatogram>}

$\mathrm{mV}$

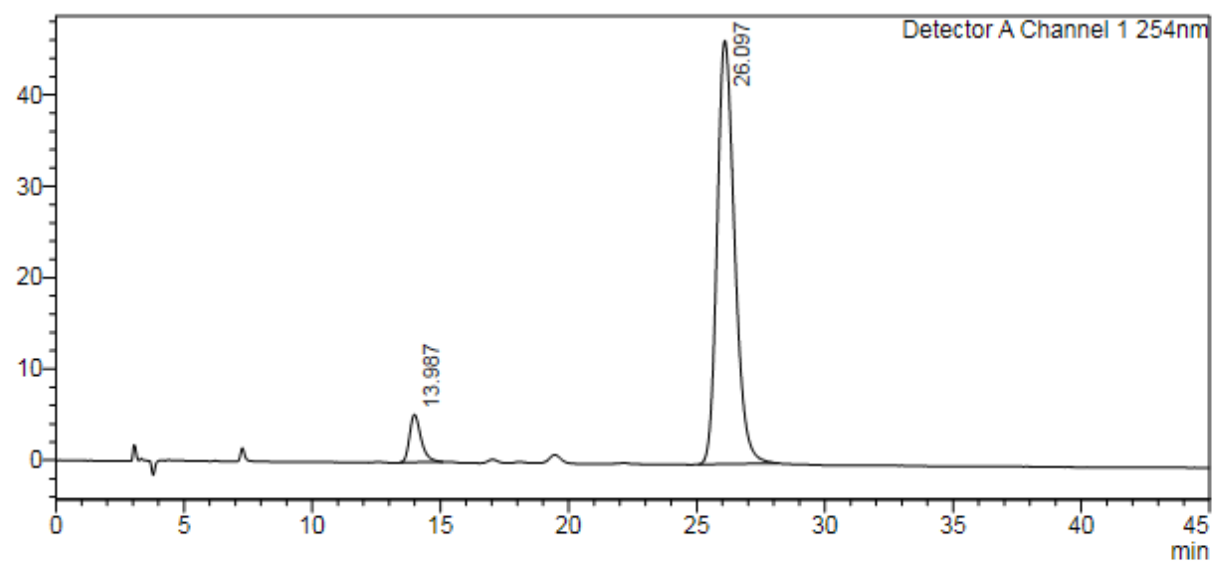

<Peak Table>

Detector A Channel $1254 \mathrm{~nm}$

Peak\# Ret. Time Area

\begin{tabular}{r|r|r|r|r|r|r|r} 
Peak\# Ret. Time & Area & Height & Conc. & Unit & Mark & Name \\
\hline 1 & 13.987 & 162378 & 5219 & 6.875 & & & \\
\hline 2 & 26.097 & 2199609 & 46337 & 93.125 & & & \\
Total & & 2361987 & 51556 & & & & \\
\hline
\end{tabular}


(-)-Brevianamide B (2) e.r. $92: 8$

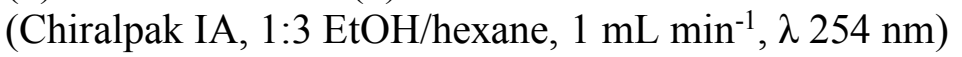

$t_{\text {Rmajor }}=13.86 \mathrm{~min}, t_{\text {Rminor }}=26.14 \mathrm{~min}$.

\section{$<$ Chromatogram $>$}

$\mathrm{mV}$

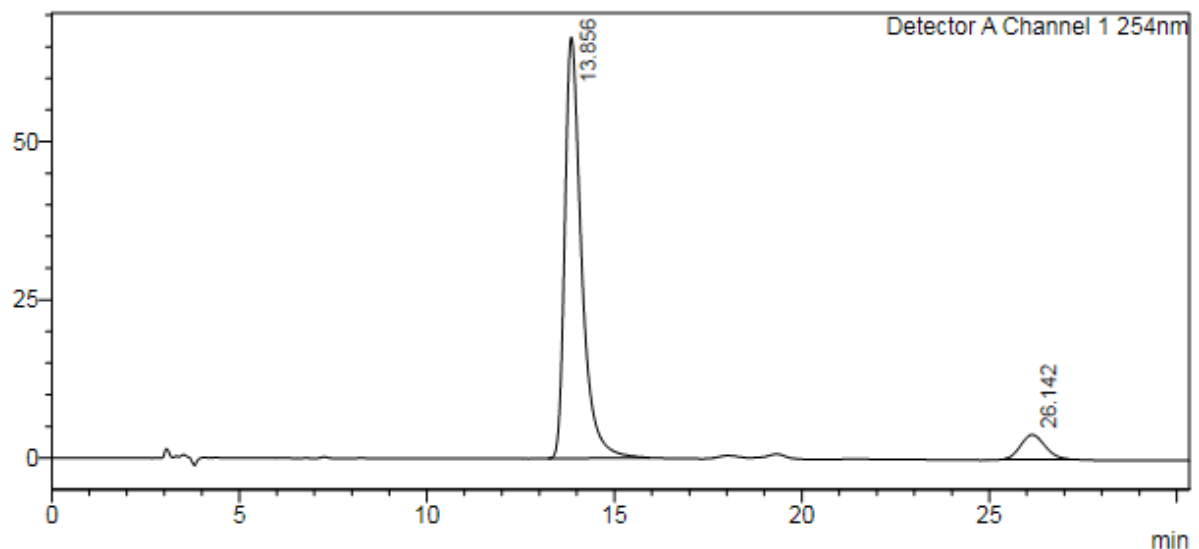

<Peak Table>

Detector A Channel $1254 \mathrm{~nm}$
Peak\# Ret. Time

\begin{tabular}{r|r|r|}
1 & 13.856 & 2058078 \\
\hline 2 & 26.142 & 180065 \\
\hline
\end{tabular}

\begin{tabular}{l|l|l|l|l} 
Height & Conc. & Unit & Mark & Name
\end{tabular}

\begin{tabular}{l|r} 
Total & 2238144 \\
\hline
\end{tabular} 
Submitted by: Robert Godfrey

The University of Edinburgh

Solved by: Gary $\mathbf{S}$ Nichol

Sample ID: $\quad$ RG-G-96

Compound RG-G-96 was provided as orange prism/plate-shaped crystals suitable for single crystal Xray diffraction, yielding structure AL19001.

\section{Crystal Data and Experimental}

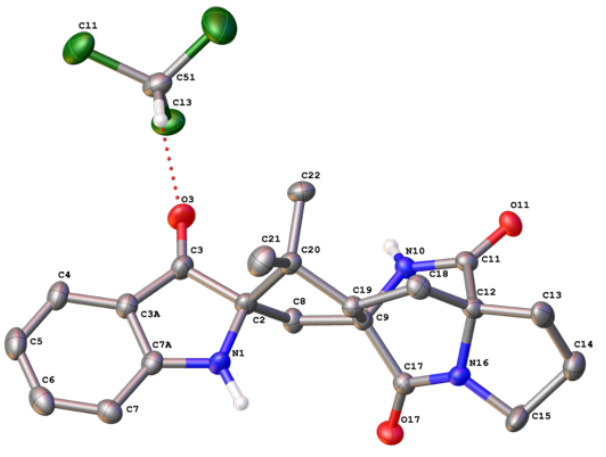

Experimental. Single yellow plate-shaped crystals of AL19001 were recrystallised from a mixture of chloroform and diethyl ether by vapour diffusion. A suitable crystal $0.24 \times 0.09 \times 0.05 \mathrm{~mm}^{3}$ was selected and mounted on a MITIGEN holder in Paratone oil on an Rigaku Oxford Diffraction SuperNova diffractometer. The crystal was kept at a steady $T=120.0 \mathrm{~K}$ during data collection. The structure was solved with the ShelXT (Sheldrick, 2015) structure solution program using the Intrinsic Phasing solution method and by using 0lex2 (Dolomanov et al., 2009) as the graphical interface. The model was refined with version 2018/3 of ShelXL (Sheldrick, 2015) using Least Squares minimisation.

Crystal Data. $\mathrm{C}_{22} \mathrm{H}_{24} \mathrm{Cl}_{3} \mathrm{~N}_{3} \mathrm{O}_{3}, M_{r}=484.79$, monoclinic, $I 2$ (No. 5), $\mathrm{a}=13.2441(2) \AA, \quad \mathrm{b}=8.65980(10) \AA, \quad \mathrm{c}=$ $20.3280(2) \AA, \quad \beta=104.2140(10)^{\circ}, \quad \alpha=\gamma=90^{\circ}, \quad V=$ $2260.07(5) \AA^{3}, T=120.0 \mathrm{~K}, Z=4, Z^{\prime}=1, \mu\left(\mathrm{CuK}_{\alpha}\right)=3.919$, 22429 reflections measured, 4696 unique $\left(R_{\text {int }}=0.0715\right)$ which were used in all calculations. The final $w R_{2}$ was 0.1135 (all data) and $R_{1}$ was 0.0434 (I > 2(I)).

\begin{tabular}{|c|c|}
\hline Compound & AL19001 \\
\hline Formula & $\mathrm{C}_{22} \mathrm{H}_{24} \mathrm{Cl}_{3} \mathrm{~N}_{3} \mathrm{O}_{3}$ \\
\hline$D_{\text {calc. }} / \mathrm{g} \mathrm{cm}^{-3}$ & 1.425 \\
\hline$\mu / \mathrm{mm}^{-1}$ & 3.919 \\
\hline Formula Weight & 484.79 \\
\hline Colour & yellow \\
\hline Shape & plate \\
\hline $\mathrm{Size} / \mathrm{mm}^{3}$ & $0.24 \times 0.09 \times 0.05$ \\
\hline$T / \mathrm{K}$ & 120.0 \\
\hline Crystal System & monoclinic \\
\hline Flack Parameter & $-0.007(8)$ \\
\hline Hooft Parameter & $-0.004(5)$ \\
\hline Space Group & $I 2$ \\
\hline$a / \AA$ & $13.2441(2)$ \\
\hline$b / \AA$ & $8.65980(10)$ \\
\hline$c / \AA$ & $20.3280(2)$ \\
\hline$\alpha /^{\circ}$ & 90 \\
\hline$\beta /^{\circ}$ & $104.2140(10)$ \\
\hline$\gamma /^{\circ}$ & 90 \\
\hline $\mathrm{V} / \AA^{3}$ & $2260.07(5)$ \\
\hline$Z$ & 4 \\
\hline$Z^{\prime}$ & 1 \\
\hline Wavelength/Å & 1.54184 \\
\hline Radiation type & $\mathrm{CuK}_{\alpha}$ \\
\hline$\Theta_{\min } /^{\circ}$ & 3.618 \\
\hline$\Theta_{\max } /^{\circ}$ & 76.076 \\
\hline Measured Refl. & 22429 \\
\hline Independent Refl. & 4696 \\
\hline $\begin{array}{l}\text { Reflections with I > } \\
2(\mathrm{I})\end{array}$ & 4622 \\
\hline$R_{\text {int }}$ & 0.0715 \\
\hline Parameters & 301 \\
\hline Restraints & 12 \\
\hline Largest Peak & 0.283 \\
\hline Deepest Hole & -0.377 \\
\hline GooF & 1.040 \\
\hline$w R_{2}$ (all data) & 0.1135 \\
\hline$w R_{2}$ & 0.1130 \\
\hline$R_{1}$ (all data) & 0.0437 \\
\hline$R_{1}$ & 0.0434 \\
\hline
\end{tabular}




\section{Structure Quality Indicators}

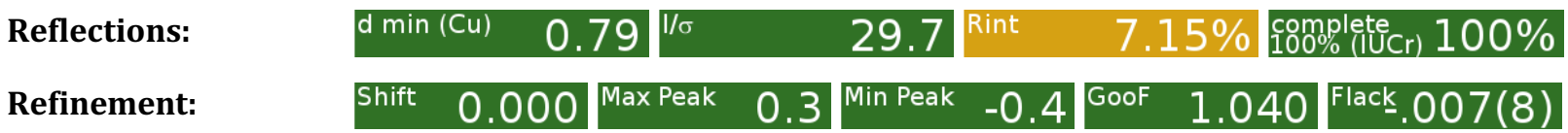

A yellow plate-shaped crystal with dimensions $0.24 \times 0.09 \times 0.05 \mathrm{~mm}^{3}$ was mounted on a MITIGEN holder in Paratone oil. Data were collected using an Rigaku Oxford Diffraction SuperNova diffractometer equipped with an Oxford Cryosystems Cryostream 700+low-temperature device operating at $T=120.0 \mathrm{~K}$.

Data were measured using $\omega$ scans using $\mathrm{CuK}_{\alpha}$ radiation. The total number of runs and images was based on the strategy calculation from the program CrysAlisPro (Rigaku, V1.171.39.46, 2018) The maximum resolution that was achieved was $\Theta=76.076^{\circ}(0.79 \AA)$.

The diffraction pattern was indexed The total number of runs and images was based on the strategy calculation from the program CrysAlisPro (Rigaku, V1.171.39.46, 2018) and the unit cell was refined using CrysAlisPro (Rigaku, V1.171.39.46, 2018) on 18841 reflections, 84\% of the observed reflections.

Data reduction, scaling and absorption corrections were performed using CrysAlisPro (Rigaku, V1.171.39.46, 2018). The final completeness is $100.00 \%$ out to $76.076^{\circ}$ in $\Theta$. A multi-scan absorption correction was performed using CrysAlisPro 1.171.39.46 (Rigaku Oxford Diffraction, 2018) using spherical harmonicsas implemented in SCALE3 ABSPACK.. The absorption coefficient $\mu$ of this material is $3.919 \mathrm{~mm}^{-1}$ at this wavelength $(\lambda=1.542 \AA)$ and the minimum and maximum transmissions are 0.732 and 1.000 .

The structure was solved and the space group I2 (\# 5) determined by the ShelXT (Sheldrick, 2015) structure solution program using Intrinsic Phasing and refined by Least Squares using version 2018/3 of ShelXL (Sheldrick, 2015). All non-hydrogen atoms were refined anisotropically. Hydrogen atom positions were calculated geometrically and refined using the riding model. Most hydrogen atom positions were calculated geometrically and refined using the riding model, but some hydrogen atoms were refined freely.

_refine_special_details: $\mathrm{N}$-bound $\mathrm{H}$ atoms were identified from a difference Fourier map and freely refined. C14 was modelled as disordered over two sites consistent with a residual peak in a difference map. Geometric and displacement ellipsoid restraints were used

The Flack parameter was refined to -0.007(8). Determination of absolute structure using Bayesian statistics on Bijvoet differences using the Olex2 results in -0.004(5). Note: The Flack parameter is used to determine chirality of the crystal studied, the value should be near 0 , a value of 1 means that the stereochemistry is wrong and the model should be inverted. A value of 0.5 means that the crystal consists of a racemic mixture of the two enantiomers.

\section{Data Plots: Diffraction Data}
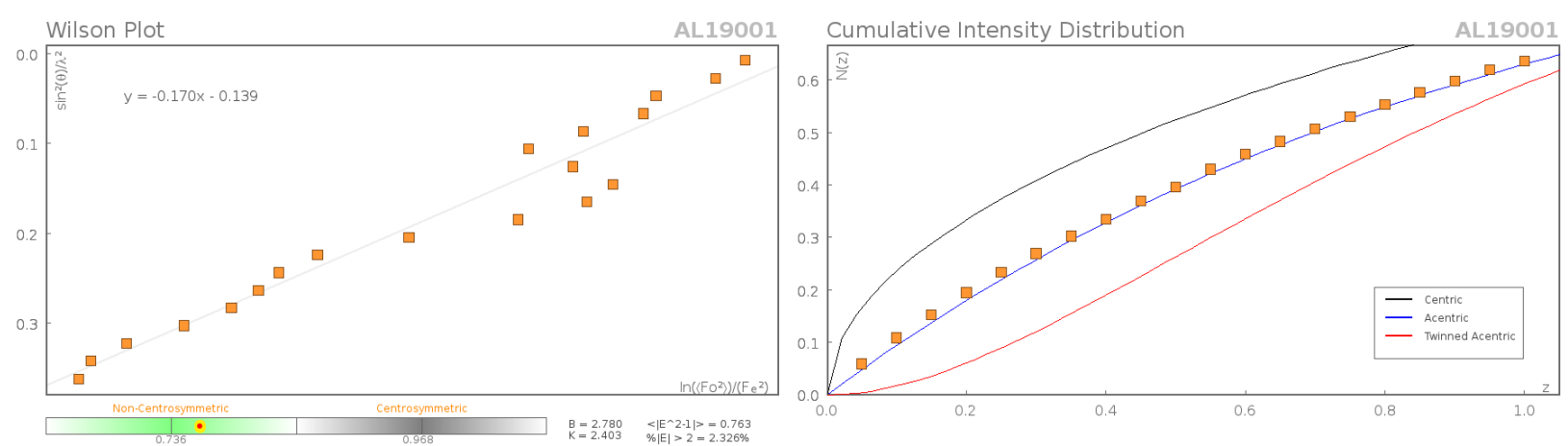

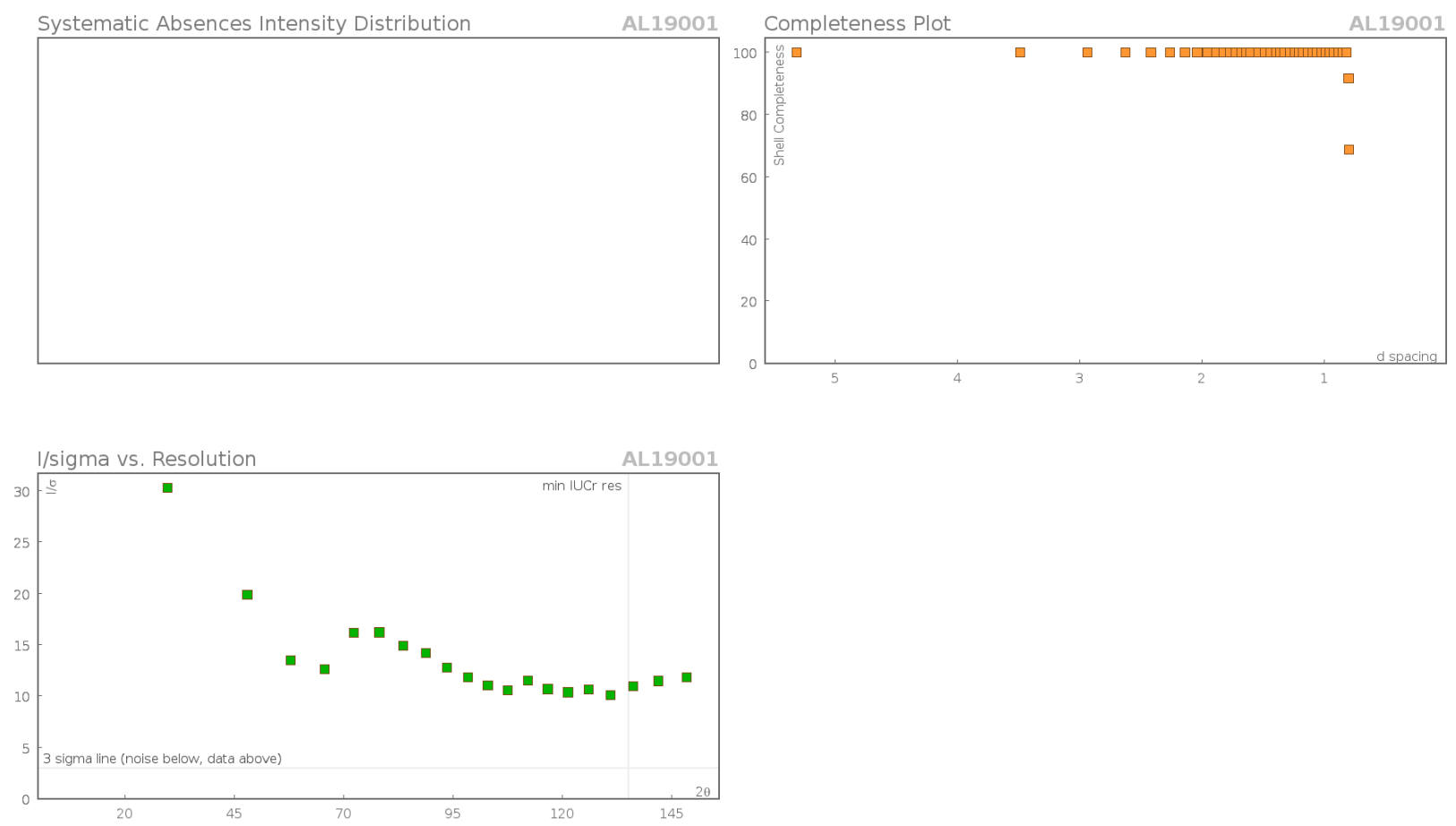

\section{Data Plots: Refinement and Data}
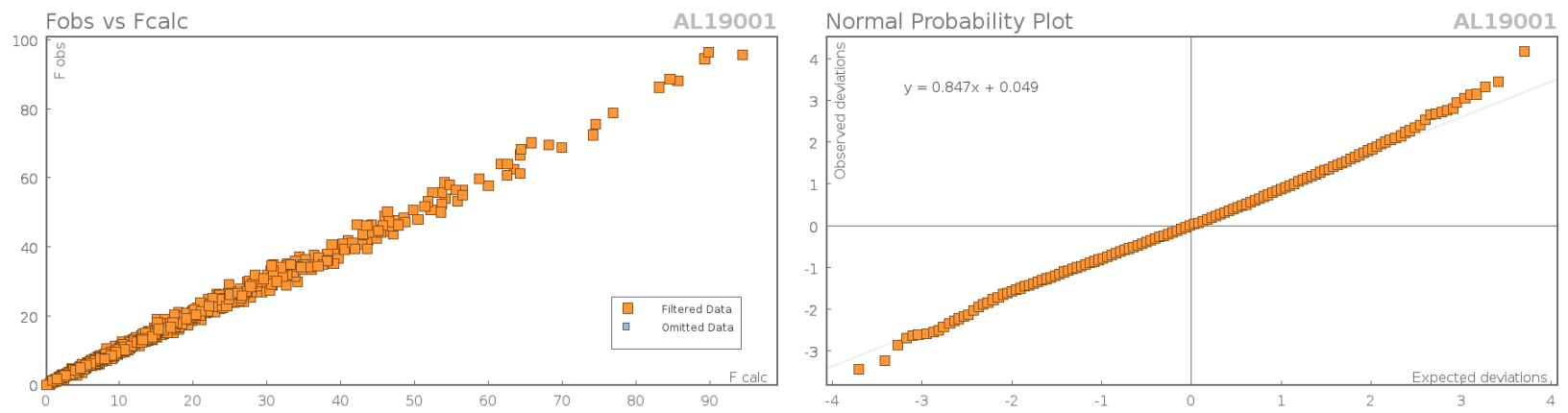

\section{Reflection Statistics}

\begin{tabular}{|c|c|c|c|}
\hline $\begin{array}{l}\text { Total reflections (after } \\
\text { filtering) }\end{array}$ & 22429 & Unique reflections & 4696 \\
\hline Completeness & 0.992 & Mean I/ $\sigma$ & 22.37 \\
\hline $\mathrm{hkl}_{\max }$ collected & $(16,10,24)$ & $\mathrm{hkl}_{\min }$ collected & $(-16,-10,-25)$ \\
\hline $\mathrm{hkl}_{\max }$ used & $(16,10,25)$ & $\mathrm{hkl}_{\min }$ used & $(-16,-10,0)$ \\
\hline Lim $d_{\max }$ collected & 100.0 & Lim $\mathrm{d}_{\min }$ collected & 0.77 \\
\hline $\mathrm{d}_{\max }$ used & 12.22 & $\mathrm{~d}_{\min }$ used & 0.79 \\
\hline Friedel pairs & 3373 & Friedel pairs merged & 0 \\
\hline Inconsistent equivalents & 39 & $\mathrm{R}_{\text {int }}$ & 0.0715 \\
\hline $\mathrm{R}_{\text {sigma }}$ & 0.0337 & Intensity transformed & 0 \\
\hline Omitted reflections & 0 & Omitted by user (OMIT hkl) & 0 \\
\hline Multiplicity & $\begin{array}{l}(2018,2488,1509,896,509, \\
293,113,66,60,54,32,17,4 \text {, } \\
\text { 1) }\end{array}$ & Maximum multiplicity & 16 \\
\hline Removed systematic abse & & Filtered off (Shel/OMIT) & 0 \\
\hline
\end{tabular}

Table 1: Fractional Atomic Coordinates $\left(\times 10^{4}\right)$ and Equivalent Isotropic Displacement Parameters $\left(\AA^{2} \times 10^{3}\right)$ for AL19001. $U_{e q}$ is defined as $1 / 3$ of the trace of the orthogonalised $U_{i j}$.

\begin{tabular}{llrrr}
\hline Atom & \multicolumn{1}{c}{$\mathbf{x}$} & $\mathbf{y}$ & $\mathbf{z}$ & $\boldsymbol{U}_{\text {eq }}$ \\
\hline $\mathrm{Cl} 1$ & $5403.6(6)$ & $10543.2(12)$ & $957.1(4)$ & $45.8(2)$ \\
$\mathrm{Cl} 2$ & $4150.5(11)$ & $7793.6(15)$ & $635.0(6)$ & $69.2(4)$
\end{tabular}




\begin{tabular}{llccc}
\hline Atom & \multicolumn{1}{c}{$\mathbf{x}$} & $\mathbf{y}$ & $\mathbf{z}$ & $\boldsymbol{U}_{\text {eq }}$ \\
\hline C13 & $3665.4(6)$ & $10071.0(11)$ & $1550.5(4)$ & $41.5(2)$ \\
C51 & $4662(2)$ & $9167(4)$ & $1266.6(15)$ & $34.0(6)$ \\
O3 & $5568.8(19)$ & $7723(3)$ & $2669.1(11)$ & $36.9(5)$ \\
O11 & $2503.3(16)$ & $1574(2)$ & $2500.9(11)$ & $26.4(4)$ \\
O17 & $3852.8(16)$ & $4056(3)$ & $4699.3(10)$ & $29.8(4)$ \\
N1 & $6559.5(18)$ & $5817(3)$ & $4225.4(11)$ & $26.7(5)$ \\
N10 & $3504.9(17)$ & $3609(2)$ & $3018.7(11)$ & $20.9(4)$ \\
N16 & $3939.4(18)$ & $1792(3)$ & $4142.0(11)$ & $23.1(5)$ \\
C2 & $5805(2)$ & $5759(3)$ & $3567.3(12)$ & $23.4(5)$ \\
C3 & $6069(2)$ & $7250(3)$ & $3219.7(14)$ & $26.5(5)$ \\
C3A & $6966(2)$ & $7942(3)$ & $3680.1(14)$ & $24.9(5)$ \\
C4 & $7527(2)$ & $9290(3)$ & $3620.3(16)$ & $29.1(6)$ \\
C5 & $8325(2)$ & $9736(4)$ & $4167.3(18)$ & $35.3(6)$ \\
C6 & $8552(2)$ & $8836(4)$ & $4760.7(16)$ & $32.2(6)$ \\
C7 & $8008(2)$ & $7506(4)$ & $4827.0(14)$ & $27.3(5)$ \\
C7A & $7192(2)$ & $7061(3)$ & $4272.6(14)$ & $23.5(5)$ \\
C8 & $4665.0(19)$ & $5717(3)$ & $3638.1(13)$ & $23.4(5)$ \\
C9 & $4365.4(19)$ & $4010(3)$ & $3612.1(12)$ & $19.6(5)$ \\
C11 & $3239(2)$ & $2112(3)$ & $2940.0(12)$ & $21.5(5)$ \\
C12 & $4003(2)$ & $1148(3)$ & $3472.1(13)$ & $23.0(5)$ \\
C13 & $3702(2)$ & $-526(3)$ & $3521.8(14)$ & $30.2(6)$ \\
C14 & $2973(4)$ & $-500(5)$ & $3972(2)$ & $36.3(11)$ \\
C15 & $3492(3)$ & $696(4)$ & $4537.2(17)$ & $36.7(7)$ \\
C17 & $4024(2)$ & $3337(3)$ & $4222.6(13)$ & $22.0(5)$ \\
C18 & $5096(2)$ & $1458(3)$ & $3355.3(13)$ & $24.6(5)$ \\
C19 & $5368.9(19)$ & $3125(3)$ & $3569.7(12)$ & $20.5(5)$ \\
C20 & $5928(2)$ & $4224(3)$ & $3178.2(13)$ & $23.3(5)$ \\
C21 & $7064(2)$ & $3757(4)$ & $3255.3(17)$ & $34.7(6)$ \\
C22 & $5389(2)$ & $4348(4)$ & $2418.1(13)$ & $30.5(6)$ \\
C14A & $3891(9)$ & $-875(12)$ & $4304(5)$ & $26(3)$ \\
& & & &
\end{tabular}

Table 2: Anisotropic Displacement Parameters $\left(\times 10^{4}\right)$ AL19001. The anisotropic displacement factor exponent takes the form: $-2 \pi^{2}\left[h^{2} a^{* 2} \times U_{11}+\ldots+2 h k a^{*} \times b^{*} \times U_{12}\right]$

\begin{tabular}{|c|c|c|c|c|c|c|}
\hline Atom & $U_{11}$ & $U_{22}$ & $U_{33}$ & $\boldsymbol{U}_{23}$ & $U_{13}$ & $U_{12}$ \\
\hline $\mathrm{Cl} 1$ & $29.5(3)$ & $62.4(6)$ & $46.8(4)$ & $16.5(4)$ & $11.7(3)$ & $-3.2(3)$ \\
\hline $\mathrm{Cl} 2$ & $85.4(8)$ & $62.6(7)$ & $67.8(6)$ & $-27.0(6)$ & $34.6(6)$ & $-18.1(6)$ \\
\hline $\mathrm{Cl} 3$ & $36.2(4)$ & $58.8(5)$ & $33.6(3)$ & $-0.8(3)$ & $16.4(3)$ & $0.7(3)$ \\
\hline C51 & $34.4(14)$ & $41.9(16)$ & $27.0(13)$ & $5.8(12)$ & $10.3(11)$ & $0.8(13)$ \\
\hline 03 & $42.3(12)$ & $35.4(12)$ & $27.3(9)$ & $13.2(9)$ & $-2.4(9)$ & $-4.3(9)$ \\
\hline 011 & $27.0(9)$ & 21.8(8) & $27.5(8)$ & $-3.7(7)$ & $1.4(7)$ & $-1.0(7)$ \\
\hline 017 & $34.1(10)$ & $30.3(10)$ & $27.5(9)$ & $-7.2(8)$ & $12.3(8)$ & $-0.5(8)$ \\
\hline N1 & $26.3(10)$ & $29.0(12)$ & 21.1(10) & $8.0(9)$ & $-1.0(8)$ & $-5.9(9)$ \\
\hline N10 & $22.4(10)$ & $18.5(10)$ & $20.3(9)$ & $-0.8(8)$ & $2.2(8)$ & $0.6(8)$ \\
\hline N16 & $28.7(10)$ & $22.2(11)$ & $19.0(10)$ & $0.6(8)$ & $6.8(8)$ & $-2.4(8)$ \\
\hline $\mathrm{C} 2$ & $25.3(12)$ & $23.1(12)$ & $19.8(11)$ & $4.3(9)$ & $1.4(9)$ & $-1.3(10)$ \\
\hline C3 & $30.4(13)$ & $22.2(12)$ & $26.8(12)$ & $5.4(10)$ & $7.0(10)$ & $-1.6(10)$ \\
\hline C3A & $24.5(12)$ & $23.1(12)$ & $27.9(12)$ & $1.0(10)$ & $7.9(10)$ & $-1.2(10)$ \\
\hline $\mathrm{C} 4$ & $29.1(13)$ & $23.0(12)$ & $38.9(14)$ & $1.3(11)$ & $15.3(11)$ & $-2.8(11)$ \\
\hline C5 & $31.0(13)$ & $29.2(14)$ & $49.8(17)$ & $-5.9(12)$ & $17.9(13)$ & $-8.5(11)$ \\
\hline C6 & $22.5(12)$ & $35.8(15)$ & $38.6(15)$ & $-11.0(12)$ & 7.7(11) & $-2.5(11)$ \\
\hline C7 & $23.6(12)$ & $31.1(13)$ & $26.0(12)$ & $-3.6(10)$ & $4.0(10)$ & $1.5(10)$ \\
\hline C7A & $21.5(11)$ & $23.6(12)$ & $27.2(12)$ & $0.9(10)$ & $9.6(10)$ & $-0.2(9)$ \\
\hline C8 & 23.1(11) & $17.7(11)$ & $28.1(12)$ & $0.3(10)$ & $3.8(9)$ & $-0.5(9)$ \\
\hline C9 & $21.3(11)$ & $17.1(10)$ & $19.8(11)$ & $-0.9(8)$ & $3.9(9)$ & $0.0(9)$ \\
\hline C11 & $25.8(12)$ & $19.7(11)$ & $18.9(11)$ & $-2.1(9)$ & $5.4(9)$ & $1.2(9)$ \\
\hline C12 & $29.4(13)$ & $18.6(11)$ & $20.6(11)$ & $-1.7(9)$ & $5.0(9)$ & $0.4(9)$ \\
\hline C13 & $40.7(15)$ & $17.1(11)$ & $29.7(13)$ & $0.6(10)$ & $2.6(12)$ & $-1.1(10)$ \\
\hline C14 & $44(2)$ & $27.3(18)$ & $40(2)$ & $0.8(16)$ & $14.8(17)$ & $-9.9(16)$ \\
\hline C15 & $52.0(17)$ & $29.4(14)$ & $36.7(14)$ & $-0.8(13)$ & $25.9(13)$ & $-11.7(14)$ \\
\hline C17 & $21.3(11)$ & $22.2(12)$ & $22.0(11)$ & $-1.8(9)$ & $4.2(9)$ & $-0.4(9)$ \\
\hline C18 & $26.5(12)$ & $19.4(12)$ & $27.5(12)$ & $-2.7(10)$ & $6.0(9)$ & $4.7(10)$ \\
\hline
\end{tabular}




\begin{tabular}{lllllll}
\hline Atom & \multicolumn{1}{c}{$\boldsymbol{U}_{\mathbf{1 1}}$} & \multicolumn{1}{c}{$\boldsymbol{U}_{\mathbf{2 2}}$} & \multicolumn{1}{c}{$\boldsymbol{U}_{\mathbf{3 3}}$} & $\boldsymbol{U}_{\mathbf{2 3}}$ & $\boldsymbol{U}_{\mathbf{1 3}}$ & $\boldsymbol{U}_{\mathbf{1 2}}$ \\
\hline C19 & $21.0(11)$ & $19.5(11)$ & $20.9(10)$ & $0.5(9)$ & $5.1(8)$ & $1.4(9)$ \\
C20 & $24.2(12)$ & $23.3(12)$ & $22.7(11)$ & $2.3(9)$ & $6.2(9)$ & $0.0(9)$ \\
C21 & $26.3(13)$ & $37.4(16)$ & $42.9(16)$ & $7.0(13)$ & $13.3(11)$ & $3.6(11)$ \\
C22 & $35.8(14)$ & $35.4(14)$ & $21.0(12)$ & $1.7(11)$ & $8.1(10)$ & $-1.2(12)$ \\
C14A & $32(6)$ & $21(5)$ & $27(5)$ & $3(4)$ & $8(4)$ & $-6(4)$
\end{tabular}

Table 3: Bond Lengths in Å for AL19001.

\begin{tabular}{lll}
\hline Atom & Atom & Length/Å \\
\hline $\mathrm{Cl} 1$ & $\mathrm{C} 51$ & $1.756(3)$ \\
$\mathrm{Cl} 2$ & $\mathrm{C} 51$ & $1.759(3)$ \\
$\mathrm{Cl} 3$ & $\mathrm{C} 51$ & $1.750(3)$ \\
$\mathrm{O} 3$ & $\mathrm{C} 3$ & $1.223(4)$ \\
$\mathrm{O} 11$ & $\mathrm{C} 11$ & $1.240(3)$ \\
$\mathrm{O} 17$ & $\mathrm{C} 17$ & $1.219(3)$ \\
$\mathrm{N} 1$ & $\mathrm{C} 2$ & $1.461(3)$ \\
$\mathrm{N} 1$ & $\mathrm{C} 7 \mathrm{~A}$ & $1.354(4)$ \\
$\mathrm{N} 10$ & $\mathrm{C} 9$ & $1.483(3)$ \\
$\mathrm{N} 10$ & $\mathrm{C} 11$ & $1.342(3)$ \\
$\mathrm{N} 16$ & $\mathrm{C} 12$ & $1.493(3)$ \\
$\mathrm{N} 16$ & $\mathrm{C} 15$ & $1.460(4)$ \\
$\mathrm{N} 16$ & $\mathrm{C} 17$ & $1.349(4)$ \\
$\mathrm{C} 2$ & $\mathrm{C} 3$ & $1.552(4)$ \\
$\mathrm{C} 2$ & $\mathrm{C} 8$ & $1.552(3)$ \\
$\mathrm{C} 2$ & $\mathrm{C} 20$ & $1.575(4)$ \\
$\mathrm{C} 3$ & $\mathrm{C} 3 \mathrm{~A}$ & $1.449(4)$ \\
$\mathrm{C} 3 \mathrm{~A}$ & $\mathrm{C} 4$ & $1.406(4)$ \\
$\mathrm{C} 3 \mathrm{~A}$ & $\mathrm{C} 7 \mathrm{~A}$ & $1.394(4)$
\end{tabular}

\begin{tabular}{lll}
\hline Atom & Atom & Length/A \\
\hline C4 & C5 & $1.387(5)$ \\
C5 & C6 & $1.405(5)$ \\
C6 & C7 & $1.383(4)$ \\
C7 & C7A & $1.410(4)$ \\
C8 & C9 & $1.528(3)$ \\
C9 & C17 & $1.537(4)$ \\
C9 & C19 & $1.555(3)$ \\
C11 & C12 & $1.534(3)$ \\
C12 & C13 & $1.513(4)$ \\
C12 & C18 & $1.546(4)$ \\
C13 & C14 & $1.485(5)$ \\
C13 & C14A & $1.577(11)$ \\
C14 & C15 & $1.573(5)$ \\
C15 & C14A & $1.574(11)$ \\
C18 & C19 & $1.525(3)$ \\
C19 & C20 & $1.542(3)$ \\
C20 & C21 & $1.529(4)$ \\
C20 & C22 & $1.538(3)$
\end{tabular}

Table 4: Bond Angles in ${ }^{\circ}$ for AL19001.

\begin{tabular}{|c|c|c|c|}
\hline Atom & Atom & Atom & Angle $/^{\circ}$ \\
\hline$\overline{\mathrm{Cl} 1}$ & C51 & $\mathrm{Cl} 2$ & $110.26(16)$ \\
\hline $\mathrm{Cl} 3$ & C51 & $\mathrm{Cl} 1$ & $110.35(19)$ \\
\hline $\mathrm{Cl} 3$ & C51 & $\mathrm{Cl} 2$ & $110.90(18)$ \\
\hline C7A & N1 & $\mathrm{C} 2$ & $111.4(2)$ \\
\hline C11 & N10 & $\mathrm{C} 9$ & $116.7(2)$ \\
\hline C15 & N16 & C12 & $112.4(2)$ \\
\hline C17 & N16 & C12 & $117.4(2)$ \\
\hline C17 & N16 & C15 & $127.6(2)$ \\
\hline N1 & $\mathrm{C} 2$ & $\mathrm{C} 3$ & $102.0(2)$ \\
\hline N1 & $\mathrm{C} 2$ & $\mathrm{C} 8$ & $112.2(2)$ \\
\hline N1 & $\mathrm{C} 2$ & $\mathrm{C} 20$ & $110.9(2)$ \\
\hline $\mathrm{C} 3$ & $\mathrm{C} 2$ & $\mathrm{C} 20$ & $114.2(2)$ \\
\hline $\mathrm{C} 8$ & $\mathrm{C} 2$ & $\mathrm{C} 3$ & $113.2(2)$ \\
\hline $\mathrm{C} 8$ & $\mathrm{C} 2$ & $\mathrm{C} 20$ & $104.4(2)$ \\
\hline 03 & $\mathrm{C} 3$ & $\mathrm{C} 2$ & $124.3(3)$ \\
\hline 03 & $\mathrm{C} 3$ & $\mathrm{C} 3 \mathrm{~A}$ & $128.8(3)$ \\
\hline C3A & $\mathrm{C} 3$ & $\mathrm{C} 2$ & $106.9(2)$ \\
\hline $\mathrm{C} 4$ & $\mathrm{C} 3 \mathrm{~A}$ & $\mathrm{C} 3$ & $130.9(3)$ \\
\hline C7A & $\mathrm{C} 3 \mathrm{~A}$ & $\mathrm{C} 3$ & $107.5(2)$ \\
\hline C7A & $\mathrm{C} 3 \mathrm{~A}$ & $\mathrm{C} 4$ & $121.5(3)$ \\
\hline $\mathrm{C} 5$ & $\mathrm{C} 4$ & $\mathrm{C} 3 \mathrm{~A}$ & $118.5(3)$ \\
\hline $\mathrm{C} 4$ & $\mathrm{C} 5$ & C6 & $119.6(3)$ \\
\hline $\mathrm{C} 7$ & C6 & $\mathrm{C} 5$ & $122.6(3)$ \\
\hline C6 & $\mathrm{C} 7$ & C7A & $117.7(3)$ \\
\hline N1 & C7A & $\mathrm{C} 3 \mathrm{~A}$ & $112.1(2)$ \\
\hline N1 & C7A & C7 & $127.9(3)$ \\
\hline C3A & C7A & C7 & $120.1(3)$ \\
\hline C9 & $\mathrm{C} 8$ & $\mathrm{C} 2$ & $105.7(2)$ \\
\hline
\end{tabular}

\begin{tabular}{llll}
\hline Atom & Atom & Atom & \multicolumn{1}{c}{ Angle ${ }^{\circ}$} \\
\hline N10 & C9 & C8 & $113.3(2)$ \\
N10 & C9 & C17 & $104.4(2)$ \\
N10 & C9 & C19 & $109.62(19)$ \\
C8 & C9 & C17 & $117.7(2)$ \\
C8 & C9 & C19 & $105.1(2)$ \\
C17 & C9 & C19 & $106.4(2)$ \\
O11 & C11 & N10 & $125.8(2)$ \\
O11 & C11 & C12 & $124.7(2)$ \\
N10 & C11 & C12 & $109.5(2)$ \\
N16 & C12 & C11 & $105.3(2)$ \\
N16 & C12 & C13 & $102.8(2)$ \\
N16 & C12 & C18 & $109.9(2)$ \\
C11 & C12 & C18 & $106.1(2)$ \\
C13 & C12 & C11 & $115.4(2)$ \\
C13 & C12 & C18 & $116.7(2)$ \\
C12 & C13 & C14A & $105.8(4)$ \\
C14 & C13 & C12 & $104.4(3)$ \\
C13 & C14 & C15 & $103.1(3)$ \\
N16 & C15 & C14 & $100.9(2)$ \\
N16 & C15 & C14A & $100.6(4)$ \\
O17 & C17 & N16 & $125.3(3)$ \\
O17 & C17 & C9 & $126.6(3)$ \\
N16 & C17 & C9 & $108.1(2)$ \\
C19 & C18 & C12 & $106.6(2)$ \\
C18 & C19 & C9 & $109.7(2)$ \\
C18 & C19 & C20 & $123.2(2)$ \\
C20 & C19 & C9 & $104.42(19)$ \\
C19 & C20 & C2 & $98.06(19)$
\end{tabular}




\begin{tabular}{lllc}
\hline Atom & Atom & Atom & Angle $^{\circ}$ \\
\hline C21 & C20 & C2 & $113.1(2)$ \\
C21 & C20 & C19 & $111.6(2)$ \\
C21 & C20 & C22 & $108.9(2)$
\end{tabular}

\begin{tabular}{llll}
\hline Atom & Atom & Atom & Angle $^{\circ}$ \\
\hline C22 & C20 & C2 & $111.5(2)$ \\
C22 & C20 & C19 & $113.5(2)$ \\
C15 & C14A & C13 & $99.0(6)$
\end{tabular}

Table 5: Torsion Angles in ${ }^{\circ}$ for AL19001.

\begin{tabular}{|c|c|c|c|c|}
\hline Atom & Atom & Atom & Atom & Angle ${ }^{\circ}$ \\
\hline 03 & C3 & $\mathrm{C} 3 \mathrm{~A}$ & $\mathrm{C} 4$ & $-2.4(5)$ \\
\hline 03 & $\mathrm{C} 3$ & $\mathrm{C} 3 \mathrm{~A}$ & C7A & $173.7(3)$ \\
\hline 011 & C11 & $\mathrm{C} 12$ & N16 & $-123.4(3)$ \\
\hline 011 & C11 & C12 & C13 & $-10.7(4)$ \\
\hline 011 & C11 & C12 & C18 & $120.2(3)$ \\
\hline N1 & $\mathrm{C} 2$ & $\mathrm{C} 3$ & 03 & $-174.4(3)$ \\
\hline N1 & $\mathrm{C} 2$ & $\mathrm{C} 3$ & $\mathrm{C} 3 \mathrm{~A}$ & $3.6(3)$ \\
\hline N1 & $\mathrm{C} 2$ & $\mathrm{C} 8$ & $\mathrm{C} 9$ & $-94.9(3)$ \\
\hline N1 & $\mathrm{C} 2$ & $\mathrm{C} 20$ & C19 & $77.9(2)$ \\
\hline N1 & $\mathrm{C} 2$ & $\mathrm{C} 20$ & $\mathrm{C} 21$ & $-39.9(3)$ \\
\hline N1 & $\mathrm{C} 2$ & $\mathrm{C} 20$ & $\mathrm{C} 22$ & $-162.9(2)$ \\
\hline N10 & $\mathrm{C} 9$ & C17 & 017 & $-117.8(3)$ \\
\hline N10 & $\mathrm{C} 9$ & C17 & N16 & $62.0(3)$ \\
\hline N10 & $\mathrm{C} 9$ & C19 & C18 & $-43.0(3)$ \\
\hline N10 & C9 & C19 & $\mathrm{C} 20$ & $90.7(2)$ \\
\hline N10 & C11 & C12 & N16 & $57.7(3)$ \\
\hline N10 & C11 & C12 & C13 & $170.4(2)$ \\
\hline N10 & C11 & C12 & C18 & $-58.8(3)$ \\
\hline N16 & C12 & C13 & C14 & $30.4(3)$ \\
\hline N16 & C12 & C13 & C14A & $-20.7(5)$ \\
\hline N16 & C12 & C18 & C19 & $-44.0(3)$ \\
\hline N16 & C15 & C14A & C13 & $-41.9(6)$ \\
\hline C2 & N1 & C7A & $\mathrm{C} 3 \mathrm{~A}$ & $-0.7(3)$ \\
\hline $\mathrm{C} 2$ & N1 & C7A & C7 & $179.7(3)$ \\
\hline $\mathrm{C} 2$ & $\mathrm{C} 3$ & $\mathrm{C} 3 \mathrm{~A}$ & $\mathrm{C} 4$ & $179.7(3)$ \\
\hline $\mathrm{C} 2$ & C3 & $\mathrm{C} 3 \mathrm{~A}$ & C7A & $-4.2(3)$ \\
\hline $\mathrm{C} 2$ & C8 & $\mathrm{C} 9$ & N10 & $-116.5(2)$ \\
\hline $\mathrm{C} 2$ & C8 & $\mathrm{C} 9$ & C17 & $121.4(2)$ \\
\hline $\mathrm{C} 2$ & $\mathrm{C} 8$ & $\mathrm{C} 9$ & C19 & $3.2(2)$ \\
\hline C3 & $\mathrm{C} 2$ & C8 & C9 & $150.2(2)$ \\
\hline C3 & $\mathrm{C} 2$ & $\mathrm{C} 20$ & C19 & $-167.5(2)$ \\
\hline C3 & $\mathrm{C} 2$ & $\mathrm{C} 20$ & $\mathrm{C} 21$ & $74.8(3)$ \\
\hline C3 & $\mathrm{C} 2$ & $\mathrm{C} 20$ & $\mathrm{C} 22$ & $-48.3(3)$ \\
\hline C3 & $\mathrm{C} 3 \mathrm{~A}$ & $\mathrm{C} 4$ & $\mathrm{C} 5$ & $175.9(3)$ \\
\hline C3 & $\mathrm{C} 3 \mathrm{~A}$ & C7A & N1 & $3.2(3)$ \\
\hline C3 & $\mathrm{C} 3 \mathrm{~A}$ & C7A & $\mathrm{C} 7$ & $-177.2(2)$ \\
\hline C3A & $\mathrm{C} 4$ & $\mathrm{C} 5$ & C6 & $0.2(4)$ \\
\hline C4 & $\mathrm{C} 3 \mathrm{~A}$ & C7A & N1 & $179.8(3)$ \\
\hline $\mathrm{C} 4$ & $\mathrm{C} 3 \mathrm{~A}$ & C7A & $\mathrm{C} 7$ & $-0.7(4)$ \\
\hline $\mathrm{C} 4$ & $\mathrm{C} 5$ & $\mathrm{C} 6$ & $\mathrm{C} 7$ & $-0.1(5)$ \\
\hline C5 & C6 & $\mathrm{C} 7$ & C7A & $-0.3(4)$ \\
\hline C6 & $\mathrm{C} 7$ & C7A & N1 & $-179.8(3)$ \\
\hline C6 & $\mathrm{C} 7$ & C7A & $\mathrm{C} 3 \mathrm{~A}$ & $0.7(4)$ \\
\hline C7A & N1 & $\mathrm{C} 2$ & C3 & $-1.8(3)$ \\
\hline C7A & N1 & $\mathrm{C} 2$ & C8 & $-123.3(2)$ \\
\hline C7A & N1 & $\mathrm{C} 2$ & $\mathrm{C} 20$ & $120.2(2)$ \\
\hline C7A & $\mathrm{C} 3 \mathrm{~A}$ & $\mathrm{C} 4$ & $\mathrm{C} 5$ & $0.2(4)$ \\
\hline C8 & $\mathrm{C} 2$ & C3 & 03 & $-53.6(4)$ \\
\hline C8 & $\mathrm{C} 2$ & $\mathrm{C} 3$ & $\mathrm{C} 3 \mathrm{~A}$ & $124.4(2)$ \\
\hline $\mathrm{C} 8$ & $\mathrm{C} 2$ & $\mathrm{C} 20$ & C19 & $-43.2(2)$ \\
\hline $\mathrm{C} 8$ & $\mathrm{C} 2$ & $\mathrm{C} 20$ & $\mathrm{C} 21$ & $-161.0(2)$ \\
\hline $\mathrm{C} 8$ & $\mathrm{C} 2$ & $\mathrm{C} 20$ & $\mathrm{C} 22$ & $76.0(3)$ \\
\hline $\mathrm{C} 8$ & $\mathrm{C} 9$ & C17 & 017 & $8.8(4)$ \\
\hline
\end{tabular}




\begin{tabular}{|c|c|c|c|c|}
\hline Atom & Atom & Atom & Atom & Angle/ ${ }^{\circ}$ \\
\hline$\overline{\mathrm{C} 8}$ & C9 & C17 & N16 & $-171.4(2)$ \\
\hline C8 & $\mathrm{C} 9$ & C19 & C18 & $-165.2(2)$ \\
\hline C8 & $\mathrm{C} 9$ & C19 & $\mathrm{C} 20$ & $-31.4(2)$ \\
\hline C9 & N10 & C11 & 011 & $175.4(2)$ \\
\hline C9 & N10 & C11 & C12 & $-5.7(3)$ \\
\hline C9 & C19 & $\mathrm{C} 20$ & $\mathrm{C} 2$ & $45.4(2)$ \\
\hline C9 & C19 & C20 & $\mathrm{C} 21$ & $164.2(2)$ \\
\hline C9 & C19 & $\mathrm{C} 20$ & $\mathrm{C} 22$ & $-72.3(3)$ \\
\hline C11 & N10 & C9 & C8 & $176.2(2)$ \\
\hline C11 & N10 & $\mathrm{C} 9$ & C17 & $-54.5(3)$ \\
\hline C11 & N10 & C9 & C19 & $59.2(3)$ \\
\hline C11 & C12 & C13 & C14 & $-83.7(3)$ \\
\hline C11 & $\mathrm{C} 12$ & C13 & C14A & $-134.8(5)$ \\
\hline C11 & C12 & C18 & C19 & $69.4(2)$ \\
\hline C12 & N16 & C15 & C14 & $-16.3(3)$ \\
\hline C12 & N16 & C15 & C14A & $32.6(5)$ \\
\hline C12 & N16 & C17 & 017 & $169.8(3)$ \\
\hline C12 & N16 & C17 & C9 & $-10.0(3)$ \\
\hline C12 & C13 & C14 & C15 & $-40.8(3)$ \\
\hline C12 & C13 & C14A & C15 & $39.3(6)$ \\
\hline C12 & C18 & C19 & C9 & $-16.8(3)$ \\
\hline C12 & C18 & C19 & $\mathrm{C} 20$ & $-140.2(2)$ \\
\hline C13 & C12 & C18 & C19 & $-160.4(2)$ \\
\hline C13 & C14 & C15 & N16 & $34.7(4)$ \\
\hline C15 & N16 & C12 & C11 & $113.4(3)$ \\
\hline C15 & N16 & C12 & C13 & $-7.8(3)$ \\
\hline C15 & N16 & C12 & C18 & $-132.7(3)$ \\
\hline C15 & N16 & C17 & 017 & $9.8(5)$ \\
\hline C15 & N16 & C17 & C9 & $-170.1(3)$ \\
\hline C17 & N16 & C12 & C11 & $-49.6(3)$ \\
\hline C17 & N16 & C12 & C13 & $-170.8(2)$ \\
\hline C17 & N16 & C12 & C18 & $64.3(3)$ \\
\hline C17 & N16 & C15 & C14 & $144.6(3)$ \\
\hline C17 & N16 & C15 & C14A & $-166.6(5)$ \\
\hline C17 & C9 & C19 & C18 & $69.3(2)$ \\
\hline C17 & C9 & C19 & $\mathrm{C} 20$ & $156.95(19)$ \\
\hline C18 & $\mathrm{C} 12$ & C13 & C14 & $150.7(3)$ \\
\hline C18 & $\mathrm{C} 12$ & C13 & C14A & $99.6(5)$ \\
\hline C18 & C19 & C20 & $\mathrm{C} 2$ & $171.1(2)$ \\
\hline C18 & C19 & $\mathrm{C} 20$ & $\mathrm{C} 21$ & $-70.1(3)$ \\
\hline C18 & C19 & $\mathrm{C} 20$ & $\mathrm{C} 22$ & $53.4(3)$ \\
\hline C19 & C9 & C17 & 017 & $126.3(3)$ \\
\hline C19 & C9 & C17 & N16 & $-53.9(3)$ \\
\hline $\mathrm{C} 20$ & $\mathrm{C} 2$ & C3 & 03 & $65.8(4)$ \\
\hline C20 & $\mathrm{C} 2$ & $\mathrm{C} 3$ & C3A & $-116.2(2)$ \\
\hline C20 & $\mathrm{C} 2$ & C8 & C9 & $25.3(2)$ \\
\hline
\end{tabular}

Table 6: Hydrogen Fractional Atomic Coordinates $\left(\times 10^{4}\right)$ and Equivalent Isotropic Displacement Parameters $\left(\AA^{2} \times 10^{3}\right)$ for AL19001. $U_{e q}$ is defined as $1 / 3$ of the trace of the orthogonalised $U_{i j}$.

\begin{tabular}{lllll}
\hline Atom & \multicolumn{1}{c}{$\mathbf{x}$} & \multicolumn{1}{c}{$\mathbf{y}$} & \multicolumn{1}{c}{$\mathbf{z}$} & \multicolumn{1}{c}{$\boldsymbol{U}_{\text {eq }}$} \\
\hline H51 & 5128.69 & 8622.02 & 1659.55 & 41 \\
H1 & $6570(30)$ & $5250(50)$ & $4627(18)$ & $24(8)$ \\
H10 & $3110(30)$ & $4460(50)$ & $2772(19)$ & $28(9)$ \\
H4 & 7364.24 & 9882.45 & 3214.65 & 35 \\
H5 & 8714.89 & 10643.93 & 4140.78 & 42 \\
H6 & 9101.52 & 9154.41 & 5130.9 & 39 \\
H7 & 8177.38 & 6912.99 & 5232.33 & 33 \\
H8A & 4616.39 & 6182.66 & 4074.01 & 28
\end{tabular}




\begin{tabular}{llllll}
\hline Atom & \multicolumn{1}{c}{$\mathbf{x}$} & \multicolumn{1}{c}{$\mathbf{y}$} & \multicolumn{2}{c}{$\mathbf{z}$} & $\boldsymbol{U}_{\boldsymbol{e q}}$ \\
\hline H8B & 4199.62 & 6290.87 & 3262.1 & 36 & \\
H13A & 3355.24 & -941.82 & 3068.99 & 36 & \\
H13B & 4321.47 & -1164.53 & 3720.26 & 36 & \\
H13C & 4137.02 & -1207.49 & 3313.56 & 44 \\
H13D & 2960.9 & -689.35 & 3287.26 & 44 \\
H14A & 2918.46 & -1531.99 & 4170.17 & 44 \\
H14B & 2270.92 & -154.12 & 3723.55 & 44 \\
H15A & 2968.09 & 1194.96 & 4741.07 & 44 \\
H15B & 4035.38 & 206.61 & 4899.29 & 44 \\
H15C & 2721.33 & 749.45 & 4418.23 & 29 \\
H15D & 3759.98 & 864.76 & 5030.98 & 29 \\
H18A & 5613.77 & 741.95 & 3630.73 & 25 \\
H18B & 5087.98 & 1312.67 & 2871.04 & 52 \\
H19 & 5815.11 & 3076.07 & 4043.76 & 52 \\
H21A & 7443.49 & 4612.39 & 3110.21 & \\
H21B & 7094.76 & 2848.39 & 2973.46 & 46 \\
H21C & 7383.25 & 3510.98 & 3731.58 & \\
H22A & 4703.81 & 4829.65 & 2362.55 & 46 \\
H22B & 5304.54 & 3313.25 & 2216.75 & \\
H22C & 5816.08 & 4979.4 & 2190.88 & 46 \\
H14C & 3473.8 & -1764.57 & 4392.32 & \\
H14D & 4636.59 & -1055.16 & 4521.23 &
\end{tabular}

Table 7: Hydrogen Bond information for AL19001.

\begin{tabular}{|c|c|c|c|c|c|c|}
\hline D & H & A & $\mathrm{d}(\mathrm{D}-\mathrm{H}) / \AA ̊$ & $\mathrm{~d}(\mathrm{H}-\mathrm{A}) / \AA ̊$ & $\mathrm{~d}(\mathrm{D}-\mathrm{A}) / \AA \AA$ & D-H-A/deg \\
\hline N1 & H1 & $017^{1}$ & $0.95(4)$ & $1.91(4)$ & $2.826(3)$ & $162(3)$ \\
\hline
\end{tabular}

11-x,+y,1-z

Table 8: Atomic Occupancies for all atoms that are not fully occupied in AL19001.

\begin{tabular}{lr}
\hline Atom & Occupancy \\
\hline H13A & $0.759(11)$ \\
H13B & $0.759(11)$ \\
H13C & $0.241(11)$ \\
H13D & $0.241(11)$ \\
C14 & $0.759(11)$ \\
H14A & $0.759(11)$ \\
H14B & $0.759(11)$ \\
H15A & $0.759(11)$ \\
H15B & $0.759(11)$ \\
H15C & $0.241(11)$ \\
H15D & $0.241(11)$ \\
C14A & $0.241(11)$ \\
H14C & $0.241(11)$ \\
H14D & $0.241(11)$
\end{tabular}




\section{Citations}

CrysAlisPro Software System, Rigaku Oxford Diffraction, (2018).

O.V. Dolomanov and L.J. Bourhis and R.J. Gildea and J.A.K. Howard and H. Puschmann, Olex2: A complete structure solution, refinement and analysis program, J. Appl. Cryst., (2009), 42, 339-341.

Sheldrick, G.M., Crystal structure refinement with ShelXL, Acta Cryst., (2015), C27, 3-8.

Sheldrick, G.M., ShelXT-Integrated space-group and crystal-structure determination, Acta Cryst., (2015), A71, 3-8. 


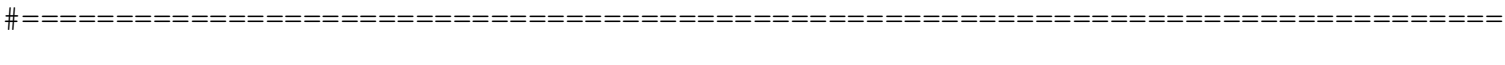

>> The Following Improvement and Query ALERTS were generated - (Acta-Mode) $<<<$

Format: alert-number_ALERT_alert-type_alert-level text

244 ALERT 4 C Low 'Solvent' Ueq as Compared to Neighbors of

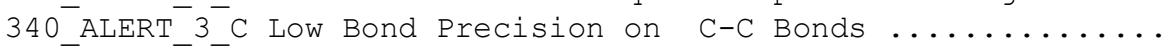

C51 Check

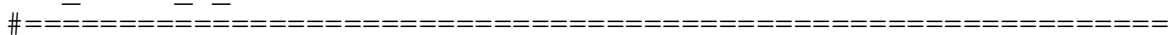
0.0041 Ang.

002 ALERT 2 G Number of Distance or Angle Restraints on AtSite $003{ }^{-}{ }_{A L E R T}{ }^{-}{ }^{-}$G Number of Uiso or Uij Restrained non-H Atoms ... 042-ALERT_1_G Calc. and Reported MoietyFormula Strings Differ 143_ALERT 4_G s.u. on c - Axis Small or Missing ............ 176_ALERT_4_G The CIF-Embedded .res File Contains SADI Records $187{ }^{-}$ALERT 4 G The CIF-Embedded .res File Contains RIGU Records $301^{-}$ALERT 3 G Main Residue Disorder ..................... 1 ) 431_ALERT_2_G Short Inter HL..A Contact C13 ..011 . $\mathrm{x}, 1+\mathrm{y}, \mathrm{z}=1565$ Check

791_ALERT 4_G Model has Chirality at C2 (Chiral SPGR) 791 ALERT 4 G Model has Chirality at C9 (Chiral SPGR) 791_ALERT4_G Model has Chirality at C12 (Chiral SPGR) 791_ALERT_4_G Model has Chirality at C19 (Chiral SPGR) $802^{-}$ALERT $^{-}$-G CIF Input Record(s) with more than 80 Characters $860^{-}$ALERT 3 G Number of Least-Squares Restraints ............ 912_ALERT_4_G Missing \# of FCF Reflections Above STh/L= 0.600 978_ALERT_2_G Number C-C Bonds with Positive Residual Density.

4 Note

3 Report

Please Check 0.00020 Ang.

2 Report

1 Report

$4 \%$ Note

3.04 Ang.

R Verify

R Verify

R Verify

S Verify

1 Info

12 Note

7 Note

3 Info

ALERT_Level and ALERT_Type Summary $======-====-==-==-=======$

2 ALERT_Level_C $=$ Check. Ensure it is Not caused by an Omission or Oversight 16 ALERT_Leve $\bar{I}_{-} G=$ General Info/Check that it is not Something Unexpected 
1 ALERT_Type_1 CIF Construction/Syntax Error, Inconsistent or Missing Data.

4 ALERT Type 2 Indicator that the Structure Model may be Wrong or Deficient.

3 ALERT_Type_3 Indicator that the structure Quality may be Low.

10 ALERT_Type_4 Improvement, Methodology, Query or Suggestion.

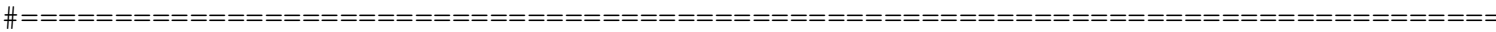

1 Missing Experimental Info Issue(s) (Out of 60 Tests) - 98 Satisfied

0 Experimental Data Related Issue(s) (Out of 30 Tests) - $100 \%$ Satisfied

7 Structural Model Related Issue(s) (Out of 131 Tests) - $95 \%$ Satisfied

10 Unresolved or to be Checked Issue(s) (Out of 257 Tests) - 96 \% Satisfied

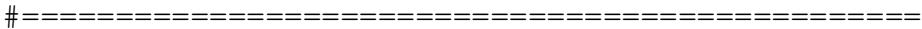

